\title{
DESIGN AND OPTIMIZATION OF COMPLIANT MECHANICAL AMPLIFIERS
}

\author{
By \\ Amiraslan Eskandari B.Eng \\ Aerospace Engineering \\ Ryerson University \\ Toronto, Ontario, Canada, 2011
}

\author{
A thesis \\ presented to Ryerson University \\ in partial fulfillment of the \\ requirements for the degree of \\ Master of Applied Science \\ in the Program of \\ Aerospace Engineering
}

Toronto, Ontario, Canada, 2013

(C) Amiraslan Eskandari 


\section{AUTHOR'S DECLARATION}

\section{AUTHORS DECLARATION FOR ELECTRONIC SUBMISSION OF A THESIS}

I hereby declare that I am the sole author of this thesis. This is a true copy of the thesis including the required final revisions, as accepted by my examiners.

I authorize Ryerson University to lend this thesis to other institutions or individuals for the purpose of scholarly research.

I further authorize Ryerson University to reproduce this thesis by photocopying or by other means, in total or in part, at request of other institutions or individuals for the purpose of scholarly research.

I understand that my thesis may be made electronically available to the public. 


\begin{abstract}
Piezoelectric (PZT) actuators are micro motion generators capable of producing a high displacement resolution and low strain with high force outputs. However, due to their relatively short motion ranges, the functions of PZT actuators become limited or infeasible for many of the above mentioned applications. One technique to overcome the mentioned shortcoming is to integrate a PZT actuator with a mechanical displacement amplifier. Such an amplification mechanism can be based on a compliant mechanical displacement amplifier (CMDA). A CMDA has many advantages such as no friction losses, no need for lubrication, no tolerance, and et al. over conventional rotating pinjoint mechanisms. Hence, the primary goal of a CMDA is to achieve a large output displacement in desired direction(s) for a given input displacement generated by a PZT actuator, and to keep a high positioning resolution at the same time.
\end{abstract}

This thesis describes a complete study on the design, development and optimization of two proposed CMDA's, one for the use in prostate Magnetic Resonance Elastography (MRE) application, and the other for the use as a planar motion generator mechanism for the application in high precision manipulation systems. The design of the both proposed mechanisms are based on the symmetric five-bar topology which facilitates a high amplification ratio (AR) with maintaining a relatively high natural frequency (NF).

The first proposed CMDA was designed and optimized to amplify the generated motion of a PZT actuator in only one direction with keeping the NF as high as possible. On the other hand, the design and optimization of the second proposed CMDA was done to convert the linear motions of the integrated PZT actuators into a XY planar motion with 
high accuracy and resolution. In this study the comprehensive FEM analysis and simulations of both proposed CMDA designs are provided. 


\section{ACKNOWLEDGMENTS}

I would like to take this opportunity to thank my supervisor Dr. Puren Ouyang for trusting me and giving me the opportunity to contribute to his research studies. Without his knowledge, guidance, encouragement, wisdom, and financial support my research would not be possible.

I would like to thank Dr. Edward J. Cunningham and Dr. Tetyana Antimirova for their extraordinary encouragement and support throughout my Undergraduate and graduate studies at Ryerson University.

I would like to extend my special thanks to Professor Zouheir Fawaz and Dr. Hamid Ghaemi for always providing me with valuable guidance and advice throughout my studies at Ryerson University. 


\section{DEDICATED TO}

My parents Farhad and Afra, and my sister Nazanin, whom without their supports and sacrifices achieving my goals would not be possible. 


\section{TABLE OF CONTENTS}

AUTHOR'S DECLARATION

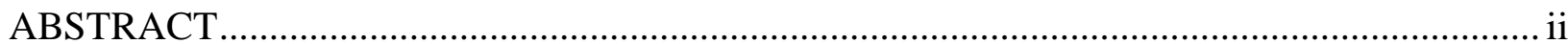

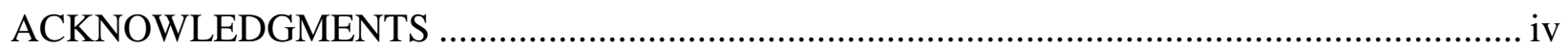

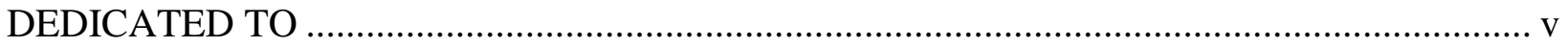

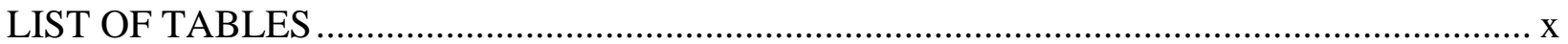

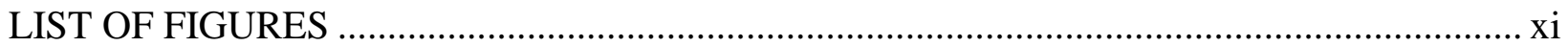

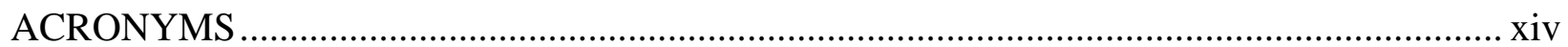

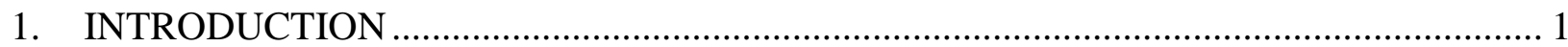

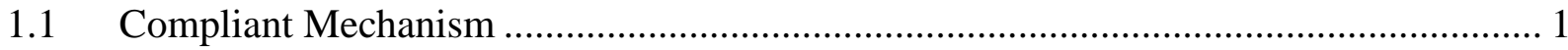

1.2 Application of Compliant Mechanisms in High Precision Manipulation Systems.......... 3

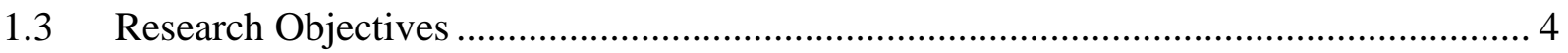

1.3.1 A Novel High Amplitude Piezoceramic Actuator for Applications in Magnetic

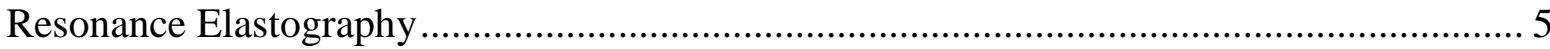

1.3.2 Design and Optimization of a XY Compliant Mechanical Displacement Amplifier 5

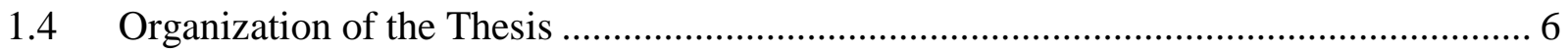

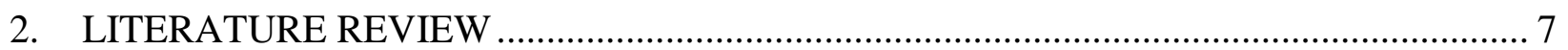

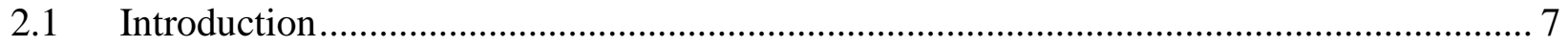

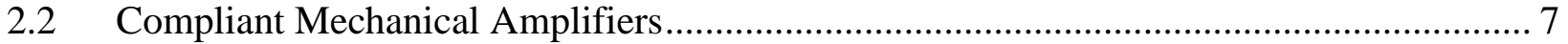

2.3 Environmental Consideration in the Design of Compliant Mechanisms ..................... 17 
2.4 Topology Optimization Techniques................................................................. 18

2.5 Topology of Symmetric Five-Bar Structure .............................................................. 22

2.5.1 Design and PRBM Analysis of the symmetric Five-Bar Structure ..................... 23

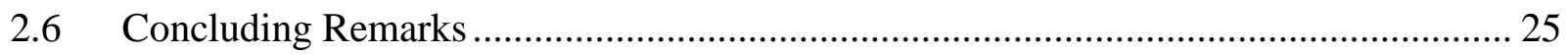

3. A NOVEL HIGH AMPLITUDE PIEZOCERAMIC ACTUATOR FOR APPLICATIONS

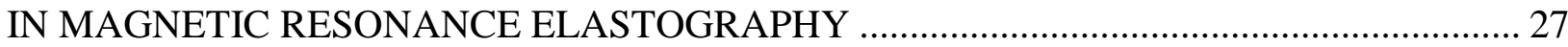

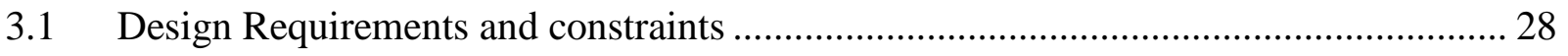

3.2 Initial FEM Static and Dynamic Analysis....................................................... 30

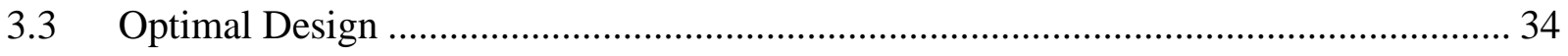

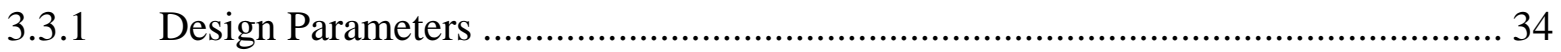

3.3.2 Optimization Process ............................................................................ 34

3.3.3 Static and Dynamic FEM Analysis on the Optimized Design (Unloaded CMDA) 37

3.3.4 Static and Dynamic FEM Analysis on the Optimized Design (Loaded CMDA for

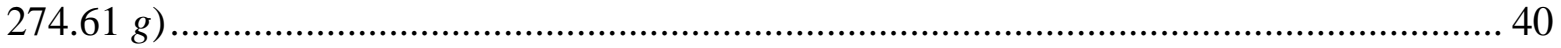

3.3.5 Experiments for Unloaded and Loaded CMDA for Prostate MRE Application .... 45

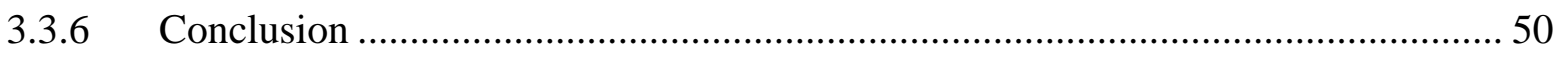

4. DESIGN AND OPTIMIZATION OF A XY COMPLIANT MECHANICAL

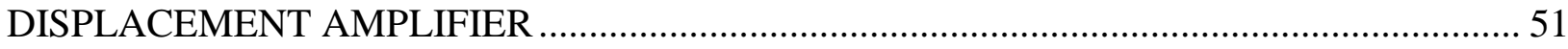

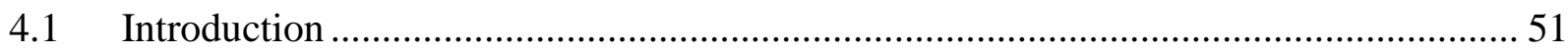

4.2 Design Requirements and Constraints ........................................................... 51 
4.3 Initial FEM Static and Dynamic Analysis........................................................ 53

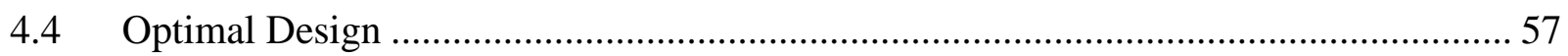

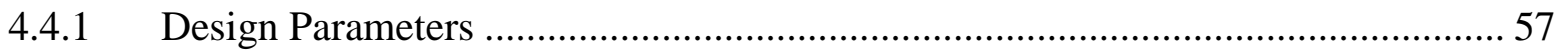

4.4.2 Optimization Process ................................................................................ 58

4.4.3 Static and Dynamic FEM Analysis on the Optimized Design............................ 60

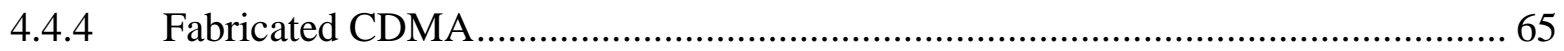

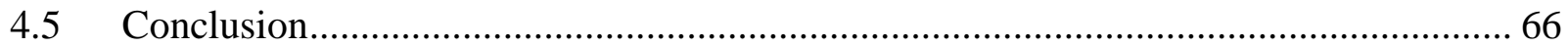

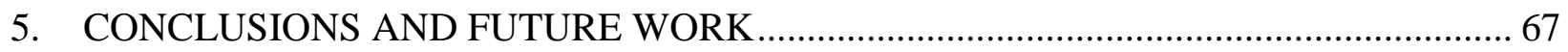

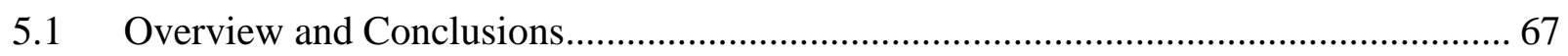

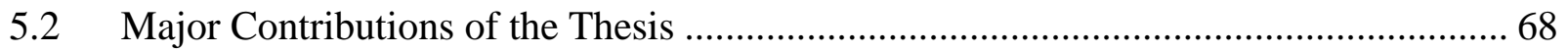

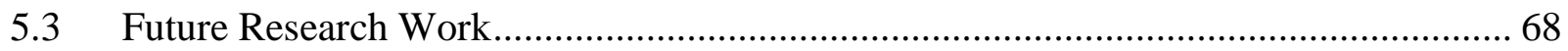

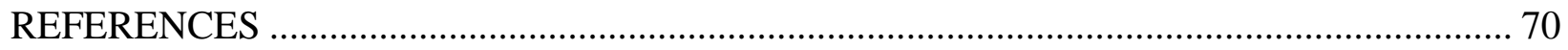

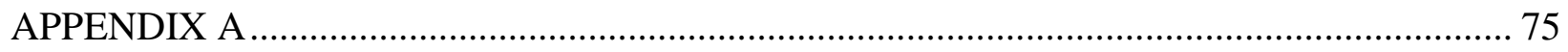

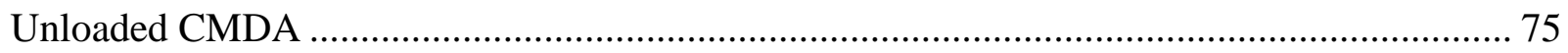

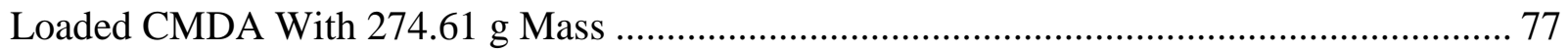

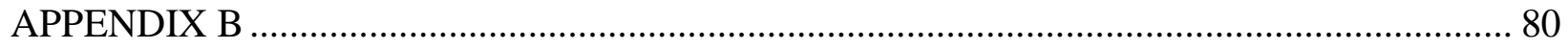

ANSYS Codes for the Unloaded CMDA.................................................................. 80

ANSYS Codes for the Loaded CMDA ...................................................................... 86

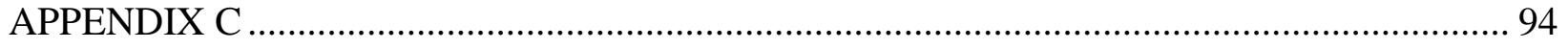

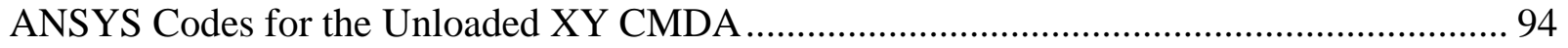


CATIA Drawing of the Proposed XY Planar Motion Generator CMDA............................. 105 


\section{LIST OF TABLES}

Table 2.1: Existed compliant mechanical amplifiers Error! Bookmark not defined.

Table 3.1: Design parameters of the proposed CMDA for the use in prostate MRE application

(unit: $\mathrm{mm}$ ) 30

Table 3.2: Brass material properties 31

Table 3.3: Initial Dynamic and Static Analysis Results 31

Table 3.4: Design Parameters and their range used for optimization (unit: mm)...................... 34

Table 3.5: Design Parameters obtained after optimization (unit: $\mathrm{mm}$ ).................................. 36

Table 3.6: Optimized Dynamic and Static Analysis Results ............................................. 38

Table 3.7: Initial Dynamic and Static Analysis Results .................................................. 41

Table 4.1: Design parameters of the planar motion generator CMDA (unit: $\mathrm{mm}$ ).................... 53

Table 4.2: Stainless Steel Material Properties ................................................................ 54

Table 4.3: Initial Dynamic and Static Analysis Results ................................................... 55

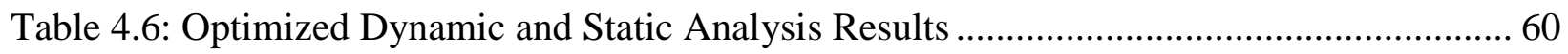




\section{LIST OF FIGURES}

Figure 1.1 Classical rotational joint (a) vs. flexure hinge $b$ (Lobontiu, 2002). .......................... 1

Figure 1.2 Classical rotational joint (a) V.S. flexure hinge $b$ (Lobontiu, 2002)......................... 1

Figure 2.1 Buckling beam topology for displacement amplification (Ouyang, Zhang, \& Gupta, 2008)

Figure2.2 Lever arm topology (Ouyang, Zhang, \& Gupta, 2008) ......................................... 11

Figure 2.3 Doubled arms topology (Ouyang, Zhang, \& Gupta, 2008).................................. 11

Figure 2.4 Parallel four-bar topology for displacement amplification (Ouyang, Zhang, \& Gupta, 2008). 12

Figure 2.5 Double symmetric four-bar topologies for displacement amplification (Ouyang, Zhang, \& Gupta, 2008).

Figure 2.6 Symmetric five-bar topologies for displacement amplification (Ouyang, Zhang, \&

Gupta, 2008). 12

Figure 2.7 The ground structure of frame members (Dinesh \& Ananthasuresh, December 12-13, 2007).

Figure 2.8 half of the resonant accelerometer with symmetry supports applied (Pedersen \&

Seshia, 2004) 21

Figure 2.9 The pseudo rigid body model of the symmetric five-bar topology (Ouyang, Zhang, \& Gupta, 2008) 23

Figure 3.1 A Photo of the mechanically coupled piezoceramic actuator ( (Arani, Plewes, Krieger,

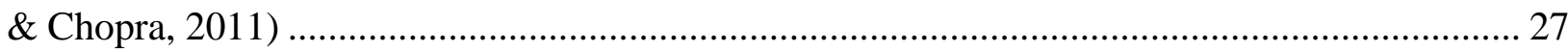

Figure 3.2 FEM model of the proposed CMDA for the use in prostate MRE application.......... 29 Figure 3.3 Key parameters used for the design of the CMDA for application in prostate MRE. 29 
Figure 3.4 Deformed shape of the initial CMDA design when $10[\mu \mathrm{m}]$ input displacement is

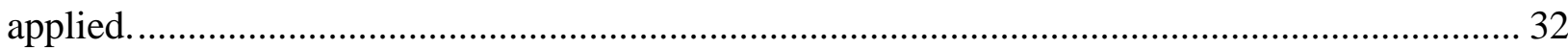

Figure 3.5. Contour plot of the Von-Mises stress of the initial CMDA. .................................. 33

Figure 3.6 Contour plot of the Von-Mises stress of the left circular flexure hinge of the initial CMDA 33

Figure 3.7 Plot of design parameters versus design objective (unit: m).............................. 37

Figure 3.8. Contour plot of the Von-Mises stress of the optimized CMDA............................. 38

Figure 3.9 Contour plot of the Von-Mises stress of the left circular flexure hinge of the optimized CMDA 39

Figure 3.10 Mode shape corresponding to the optimized unloaded CMDA with $10[\mu \mathrm{m}]$ input displacement. 40

Figure 3.11 FEM model of optimized CMDA loaded for 274.61 [ $g]$

Figure 3.12 Contour plot of the displacement vector sum of the loaded CMDA design when 10 $[\mu m]$ input displacement is applied (unit: $\mathrm{m}$ ).

Figure 3.13 Mode shape corresponding to the optimized loaded CMDA with $10[\mu \mathrm{m}]$ input displacement. 43

Figure 3.14 Contour plot of the Von-Mises stress of the loaded CMDA. 44

Figure 3.15 Contour plot of the Von-Mises stress of the right fillet corner rectangular flexure hinge of the loaded CMDA. 45

Figure 3.16 Manufactured CMDA integrated with $P I C A^{\mathrm{TM}} P-010.80 P$ PZT actuator, based on the optimized parameters obtained by FEM analysis (courtesy of Arvin Arani).. 46

Figure 3.17 Entire designed mechanism used for prostate MRE application (courtesy of Arvin Arani) 46 
Figure 3.18 displacement amplification of loaded and unladed CMDA's vs. frequency

(unpublished data, courtesy of Arvin Arani) ................................................................... 47

Figure 3.19 Displacement amplitude as a function of time. .............................................. 48

Figure 3.20 Fold amplification with CMDA (left) vs. using direct derive (right) (courtesy of

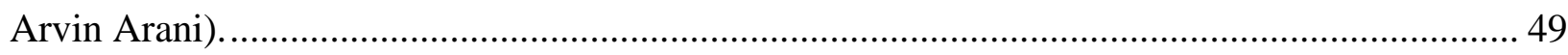

Figure 3.21 MRI image of the prostate gland with the location of stiff inclusions shown (courtesy

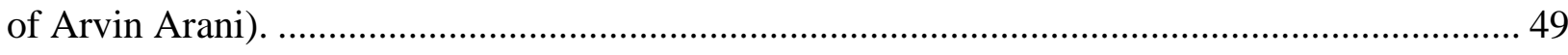

Figure 4.1 Planar motion generator CMDA based on symmetric five-bar topology.................. 52

Figure 4.2 FEM model of the planar motion generator CMDA with the locations of the applied

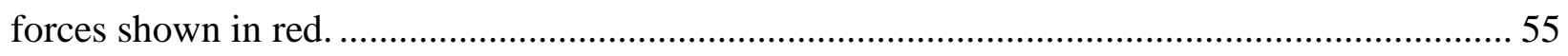

Figure 4.3 Contour plot of the Von-Mises stress of the initial CMDA. ................................... 57

Table 4.4: Design Parameters and their range used for optimization (unit: $\mathrm{mm}$ )..................... 58

Table 4.5: Design Parameters obtained after optimization (unit: $\mathrm{mm}$ ).................................. 59

Figure 4.4 Plot of design parameters versus design objective (unit: m) ............................... 60

Figure 4.5 Contour plot of the Von-Mises stress of the optimized CMDA............................. 61

Figure 4.6 Mode shape of the device corresponding to the first case ................................... 63

Figure 4.7 Mode shape of the device corresponding to the second case ................................ 64

Figure 4.8 Manufactured XY planar motion CDMA integrated with two PZT actuators ........... 65 


\section{ACRONYMS}

AR Amplification Ratio
CBT $\quad$ Compact size flexure-based compound Bridge-Type

CMA Compliant Mechanical Amplifier

CMDA Compliant Mechanical Displacement Amplifier

DOF Degree of Freedom

FEM Finite Element Method

ICAM Input Coupling Analysis Method

MRE Magnetic Resonance Elastography

MRI Magnetic Resonance Imaging

NF Natural Frequency

OBJ Objective

PRBM Pseudo-Rigid Body Model

PZT Piezo-electric

SF Safety Factor

TDPS Totally Decoupled Piezo-Driven XYZ flexure Parallel micro-positioning Stage 


\section{INTRODUCTION}

\subsection{Compliant Mechanism}

A compliant mechanism is a mechanism that its components (i.e. parts) are linked together by some flexible, slender members known as flexure hinges. The flexure hinges must undergo a relative limited rotation in the mechanism to achieve a specific task (Lobontiu, 2002). Figures 1.1 and 1.2 below show conventional rotational pin joint mechanisms in comparison to flexure hinge ones.

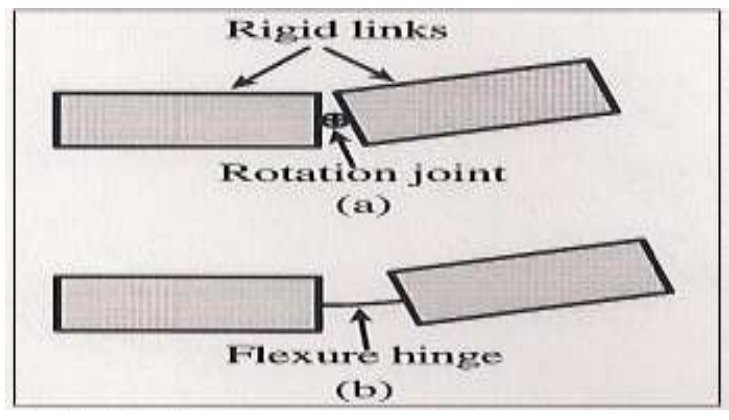

Figure 1.1 Classical rotational joint (a) vs. flexure hinge $b$ (Lobontiu, 2002).

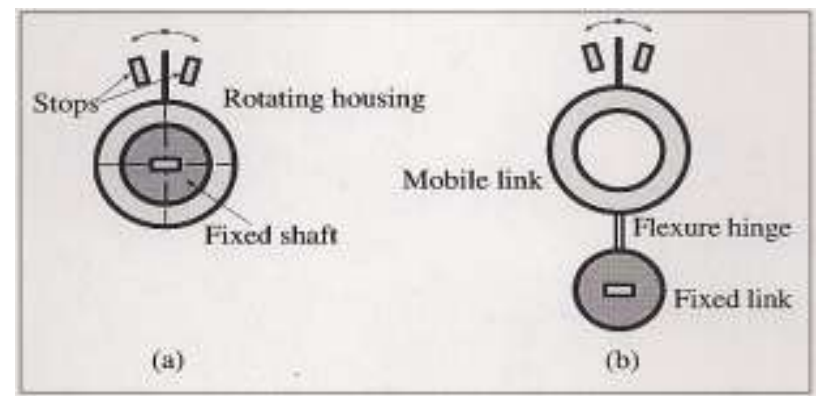

Figure 1.2 Classical rotational joint (a) V.S. flexure hinge b (Lobontiu, 2002).

As illustrated above, the flexure hinge has monolithic structure and is integrated with the rest of the mechanism. This unique feature facilitates the following advantages over the conventional rotational pin joint mechanism (Lobontiu, 2002): 
- No friction losses, hence no need for lubrication

- No hysteresis

- Compactness

- Capacity to utilize in micro scale applications

- Virtually no maintenance needed

- Ease of fabrication

Despite of the mentioned benefits provided by a compliant mechanism over classical rotational pin jointed mechanism, the flexure hinges integrated in compliant systems face some drawbacks and limitation. Few examples of such drawbacks are (Lobontiu, 2002):

- Providing of relatively low level of rotation

- The rotation is not pure due to the complex deformation of the flexure, as it is produced by axial shearing and possibly torsion loading

- The rotation center (i.e. the symmetry center of the flexure) is not fixed

- The flexure hinge is sensitive to temperature variation which results in modification in the original compliance value

In addition, the design of a compliant mechanism is more complicated than the design of a conventional rotational pin jointed mechanism, as it requires large deflection analysis, the consideration of the stored strain energy, and the solution of transcendental loop closure equations (Ü. Sönmez, 2008). 


\subsection{Application of Compliant Mechanisms in High Precision Manipulation Systems}

High precision manipulation systems have a variety of uses in many industrial applications, especially where the positioning of components with high accuracy (i.e. in micrometer or nanometer scales) is required (Yong \& Lu, 2009). Examples of these applications may include the alignment of fibre-optics and lasers, the positioning of specimens in a scanningelectron-microscope, the positioning of masks in lithography, cells manipulation in microbiology, and assembly and manipulation of micro-scale components in micro-assembly applications (Yong \& Lu, 2009).

Piezoelectric (PZT) actuators are micro motion generators capable of producing a high displacement resolution and low strain with high force outputs (Ouyang, Tjiptoprodjo, Zhang, \& Yang, 2008). However, due to their relatively short motion ranges and their ability to generate only linear displacements, the functions of PZT actuators become limited or infeasible for many of the above mentioned applications. One technique to overcome the mentioned shortcoming is to integrate a PZT actuator with a mechanical displacement amplifier (Ouyang, Zhang, \& Gupta, 2008). Such an amplification mechanism can be based on a compliant mechanical displacement amplifier (CMDA) since it benefits of many advantages (as mentioned in the previous section) facilitated with the presence of the flexure hinges in the structural design of the CMDA. Hence, the primary goal of a CMDA is to achieve a large output displacement in desired direction(s) for a given input displacement generated by a PZT actuator, and to keep a high positioning resolution at the same time. This, however, may cause a reduction in the structure's generated output force and the natural frequency (NF) of the mechanism (Ouyang, Zhang, \& Gupta). Nevertheless, the consequent 
reduction of the output force due to a CMDA can be tolerated since PZT actuators are capable of generating large amount of force, and a relatively high NF can be achieved through properly structural design. In general, the performance of a CMDA is a function of some important parameters such as the material properties and the flexure hinge profile of the mechanical displacement amplifier.

There are some other advantages of using piezoelectric actuators with mechanical displacement amplifiers with the redundancy in design. That is to say, such a system has a potential of redundancy in providing actuations in robotics. Such redundancy may be utilized for improving the resilience of the system and for improving dynamic performance of the system (Sun, Zhang, Cheng, \& Zhang, 2011). It should be mentioned that the designed CMDA can be used as the micro motion part for a Hybrid macro-micro mechanism (Ouyang, 2011). Therefore, the designed CMDA has its potential in different applications.

\subsection{Research Objectives}

This thesis study was intended to address the issues mentioned above in regards to PZT actuators and aimed to propose displacement amplification techniques, based on the compliant mechanism, with minimal compromise in the overall NF of the proposed systems. To tackle the problems of short motion range of PZT actuators and their ability of generating only linear motions, two CMDA mechanisms have been designed, optimized, manufactured, and tested as the followings: 


\subsubsection{A Novel High Amplitude Piezoceramic Actuator for Applications in Magnetic Resonance Elastography}

The objective of this study was to design a CMDA based on the design of a symmetric fivebar compliant mechanical amplifier (Ouyang, Zhang, \& Gupta, 2008) to amplify the linear output displacement of a $P I C A^{\mathrm{TM}} P-010.80 \mathrm{P}$ PZT actuator with the consideration of the NF of the mechanism. The designed CMDA in conjunction with the mentioned PZT actuator have applications in Magnetic Resonance Elastography for identifying the location of cancerous tissues in males prostate. Detailed finite element method (FEM) results of the initial and optimized designs as well as the experimental results obtained from actual testing of the mechanism are outlined in this thesis.

\subsubsection{Design and Optimization of a XY Compliant Mechanical Displacement Amplifier}

The topic of design, characteristics, and application of CMDA's is not a new one for one direction motion amplification. However, many industrial applications of high precision manipulation systems require a device capable of generating planar or special motions in the desired direction. The objective of this portion of the thesis study was motivated to design a new design for a $\mathrm{XY}$ planar CMDA based on the design of a symmetric five-bar compliant mechanical amplifier (Ouyang, Zhang, \& Gupta, 2008). Detailed FEM analysis results of the static and the dynamic characteristics of the proposed XY CMDA design are also provided. Finally, the optimization process and results to increase the Amplification Ratio (AR) of the proposed $\mathrm{XY}$ compliant mechanism with minimal compromise in NF is discussed. 


\subsection{Organization of the Thesis}

To support the research objectives provided in this thesis, the next chapter of this thesis provides a literature review on the topic of high precision micro motion manipulators and the application of compliant based mechanical amplifiers for such manipulation systems. In chapter 2 the topology of symmetric five-bar compliant mechanical amplifier (which is the base design of the two amplification mechanisms proposed in this thesis) and corresponding formulation of finding the AR and the overall structure's stiffness are also discussed. Chapters 3 and 4 present the FEM simulation and experiment results of the proposed High Amplitude Piezoceramic Actuator for Applications in Magnetic Resonance Elastography and the XY Compliant Mechanical Displacement Amplifier respectively. Finally, Chapter 5 outlines the contribution of the thesis as well as future research work. 


\section{LITERATURE REVIEW}

\subsection{Introduction}

In this chapter, the design and developments of compliant mechanisms for various industrial applications are reviewed. Section 2.2 summarizes the most common topologies of compliant mechanism, for displacement amplification purposes. In this section different flexure hinge designs, for the use within the body of compliant mechanisms, are also reviewed. Section 2.3 discusses some environmental factors that should be considered in the development and design of compliant mechanisms. The impact of the thermal effects on the performance of the compliant mechanisms is also reviewed in this section. Section 2.4 deals with the topology optimization techniques of compliant displacement amplifiers. In section 2.5 the topology of symmetric five bar structure and its corresponding pseudo-rigid body model (PRBM) formulation for finding the displacement at its output and the overall structure stiffness are discussed. Finally, some concluding remarks are given in section 2.6.

\subsection{Compliant Mechanical Amplifiers}

As it was mentioned in the preceding chapter, the direct application of PZT actuators are limited in many industrial and pharmaceutical fields (with demand of micro scale accuracy) due to piezo-material ability to generate only a small range of displacement. The most appropriate method to tackle this problem is to integrate the PZT actuator with a flexure hinged mechanical displacement amplifier which facilitates the benefits of employing compliant characteristics provided by the flexure hinges of the mechanism (Xu \& King, 
1996). In addition, the flexure based compliant micro-positioning stages are also capable of positioning with ultrahigh precision based on elastic deformations of the structures ( $\mathrm{Li} \& \mathrm{Xu}$, 2011).

In general, depending on the application, flexure-hinged displacement amplifiers can be divided into two main categories namely precision oriented and displacement oriented (Xu \& King, 1996). Xu et al (1996). listed the application of some fundamental compound amplifier topologies which combine three basic amplifying elements of simple lever, bridge, and fourbar linkage amplifiers. For example, a symmetrical structure of a bridge displacement amplifier provides it with a higher gain and linear output motion which can be employed for precision oriented applications. Alternatively, the four-bar linkage flexure-hinged amplifier is capable of generating some special output movements for both precision and high displacement oriented applications. Finally, the simple lever flexure-hinged displacement amplifier was found to be able of producing large output displacement and having the highest efficiency due to possessing less hinges than the other two topologies.

In their study, Xu et al. (1996) also talked about the effects that the geometrical profiles of flexure hinges have on the performance of the flexure-hinged displacement amplifiers. They have also analysed and compared different flexure hinge profiles such as elliptical and comer-filleted designs.

In another study, Tian et al. (2010) compared the characteristics of three flexure hinge geometries of; filleted V-shaped, cycloidal, and circular types that have applications in the design of compliant based mechanisms. In their research study, it was also tried to explore 
the influence of the geometric parameters on the characteristics of each mentioned flexure hinge designs.

Ouyang et al. (2008) discussed and compared some of the most popular topologies of compliant mechanical amplifiers (CMAs) used in conjunction with PZT actuators for micromotion applications. Figure 2.1 illustrates the topology of buckling beam structure. This topology consists of a long slender beam that buckles due to the applied axial compressive force when the force exceeds a critical value (Timoshenko \& Gere, 1961).

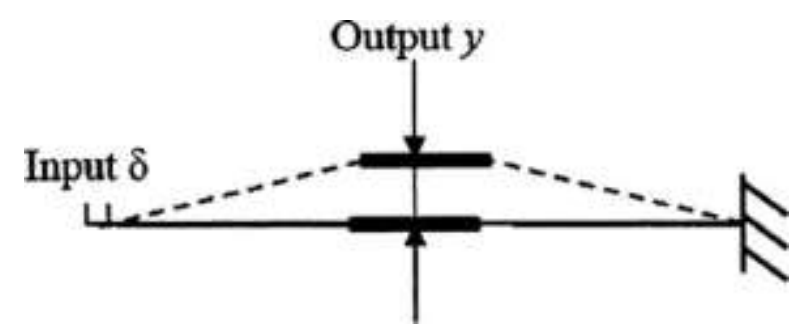

Figure 2.1 Buckling beam topology for displacement amplification (Ouyang, Zhang, \& Gupta, 2008).

Saif et al. (1998) and Jiang et al. (2003) proposed several amplifier designs based on the buckling principle. However, there are two main drawbacks associated with an amplifier developed based on the buckling beam topology. The first one is the amplifier overall larger dimensions compared to others and the second one is that the driving force should be large enough for the buckling to be achieved.

Dunning et al. (2013) presented a low stiffness 6-DOF compliant precision stage to eliminate the problems of backlash, friction and lubrication associated with conventional revolute joint mechanisms. The proposed novel compliant structure can perform ultra-precise positioning in a vacuum environment that has applications in precision engineering such as vibration isolation. The structure of the proposed mechanism takes advantage of the buckling beam topology in a unique configuration to achieve the desired tasks. The main characteristic of 
this mechanism is that all six degrees of freedom were statically balanced (i.e. near zero stiffness) to neutralize the gravity force and cancel out the stiffness due to the compliant design of the structure.

Figure 2.2 and 2.3 show the topologies of a lever arm and a multiple lever arms, respectively. The lever arm mechanism facilitates the simplest and a direct way to amplify the displacement of a PZT actuator. An amplifier developed based on the lever arm or multiple lever arms topologies can achieve a large displacement AR if no dimensional constraints are applied. The main disadvantages of this topology are the presence of a lateral displacement and amplifier's low NF and stiffness.

In a similar study, Su et al. (2001) presented the design of single stage and multistage compliant micro-leverage mechanisms. In their research the analysis of a single-stage micro leverage mechanism was introduced as the building block for the multistage micro leverage mechanisms. Also they have discussed the effects of some important parameters on the maximum displacement and force amplifications that can be achieved by single and multistage compliant micro-leverage mechanisms. In addition, it was suggested that a compound micro-lever should be designed with the minimum number of micro-lever stages to achieve the desired amplification factor. The multistage micro-lever mechanisms have applications in the design of resonant output accelerometers. A two stage micro-lever mechanism was designed and developed based on the lever arm topology in order to increase the accelerometer sensitivity by amplifying the inertial force. 


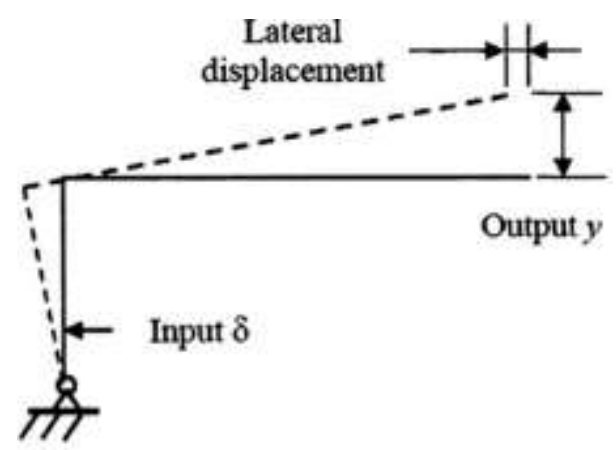

Figure2.2 Lever arm topology (Ouyang, Zhang, \& Gupta, 2008)

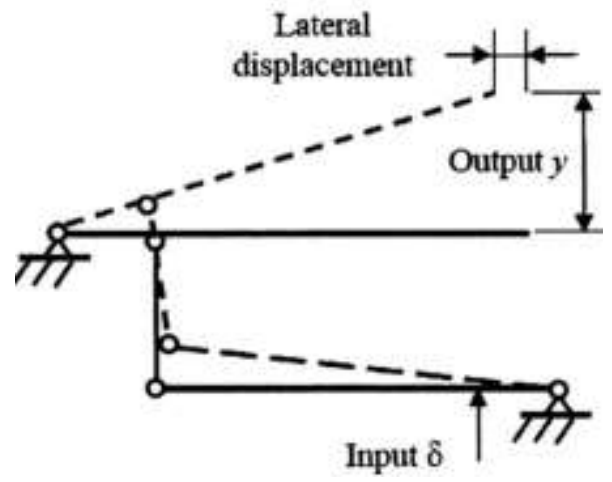

Figure 2.3 Doubled arms topology (Ouyang, Zhang, \& Gupta, 2008).

Rue et al. (1997) proposed the topology of parallel four-bar structure to improve the stiffness and consequently the NF of mechanical amplifiers. However; as shown in Figure 2.4 this topology also deals with lateral displacement problem of lever arm structure. This problem can be resolved by implementation of double symmetric four bar structures. Figure 2.5 shows the double symmetric four bar topology where the extension of the structure is driven by the input displacement $\delta$ generated by a PZT actuator. An application of this topology can be viewed in bridge displacement amplifier discussed by Xu et al. (1996). It is noticeable that amplification mechanisms based on the double symmetric four-bar topology still suffer from relatively low stiffness and low NF which may limit their industrial application. 


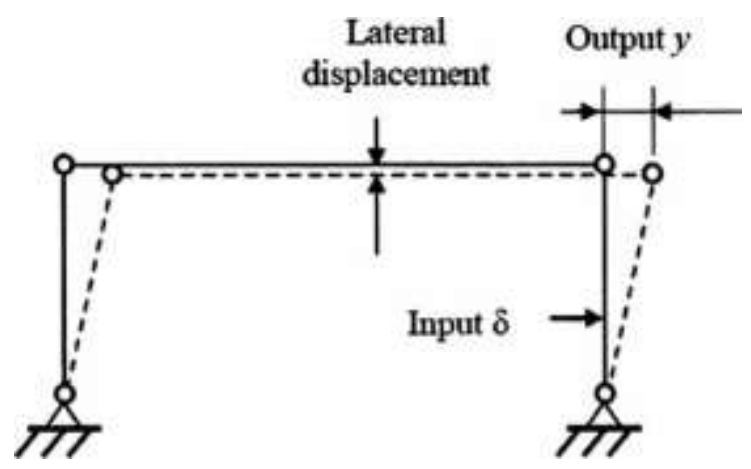

Figure 2.4 Parallel four-bar topology for displacement amplification (Ouyang, Zhang, \& Gupta, 2008).

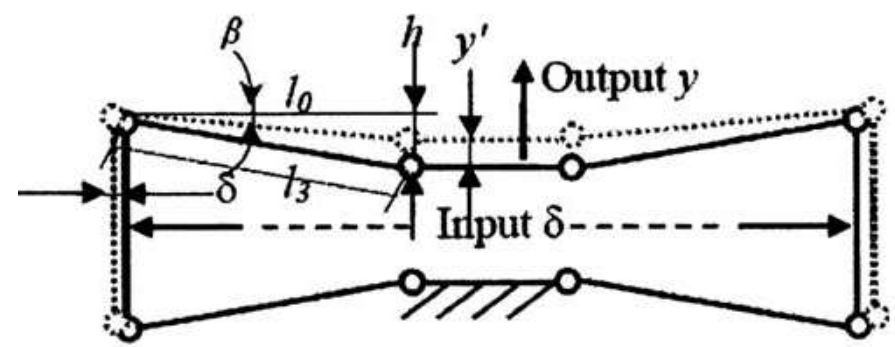

Figure 2.5 Double symmetric four-bar topologies for displacement amplification (Ouyang, Zhang, \& Gupta, 2008).

Ouyang et al. (2008) suggested the new symmetric five-bar topology which is the combination of a symmetric four-bar topology and a lever arm topology. As illustrated in Figure 2.6 the vertical bars are of lever arm structures, and between the two lever arms is a symmetric four-bar structure. The solid base on the bottom of the proposed five-bar topology can significantly increase the system stiffness and the eventual structure NF, while maintain an overall structure compact size is possible. The new topology also doesn't feature the undesired lateral displacement due to its symmetric configuration.

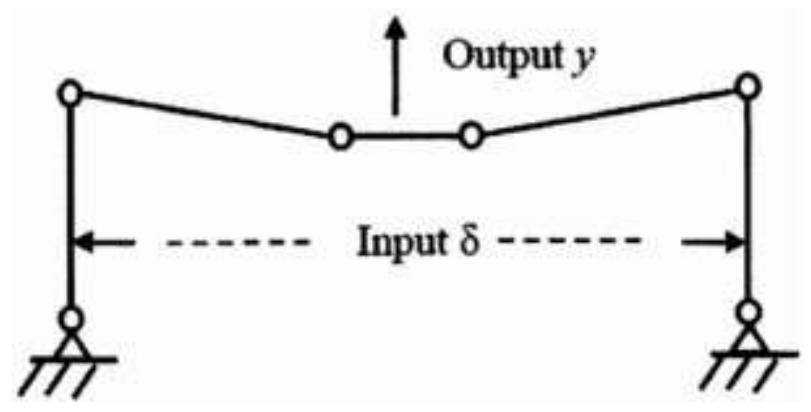

Figure 2.6 Symmetric five-bar topologies for displacement amplification (Ouyang, Zhang, \& Gupta, 2008). 
In another study Ouyang (2011) presented a spatial hybrid motion compliant mechanism based on the symmetric five-bar topology. In his study, a spatial hybrid motion mechanism with 3-DOF was developed that is capable of combining macro and micro motions through only one compliant mechanism. The integral compliant structure of the proposed mechanism helped to eliminate the coupling interaction between the macro motion and the micro motion.

Hua et al. (2008) introduced an input coupling analysis method (ICAM) for a 3-DOF compliant micro-positioning stage with a displacement amplifying mechanism. In general couplings between the input bodies of a compliant micro-positioning stage can cause serious difficulties in the motion control of the mechanism. In their study the stiffness matrix of the right circular single-axis flexure hinges, employed for the 3-DOF compliant micropositioning stage, was established, and the ICAM were developed based on the finite element theory. Their developed method was then validated by ANSYS ${ }^{\circledR}$ finite software and experimental trials. Finally, three different contact cases of PZT actuator outputs versus the input coupling of the mechanisms input were analyzed, and an optimal design method to avoid the input couplings was presented in their study.

Choi et al. (2010) proposed a novel piezo-driven compliant stage for long range for nanomotion applications. The proposed mechanism is capable of generating linear motion guide and displacement amplification. In their design they have introduced a two mechanical amplification mechanisms arranged in parallel to overcome the deterioration of the dynamics (i.e. reduction in the output force and NF) by mechanical amplification. The compliant stage of the proposed mechanism consists of two flextensional amplification mechanisms that each has eight leaf flexure hinges. 
Li and Xu (2011) designed and developed a totally decoupled piezo-driven XYZ flexure parallel micro-positioning stage (TDPS) for micro/nano-manipulation. In their research they designed and developed an XYZ TDPS with a displacement amplifier and a simple architecture for the ease of manufacturing. They have proposed a design of an XYZ stage with decoupled output motion by eliminating the cross-axis coupling errors between the $\mathrm{X}$, $\mathrm{Y}$, and $\mathrm{Z}$ directional translations and parasitic rotation errors around the axes. The right circular flexure hinges were employed for the proposed design, however; it was suggested that other hinge geometries are compatible to the mechanism and can be implemented.

In another study, $\mathrm{Li}$ and $\mathrm{Xu}$ (2011) investigated a compact size flexure-based compound bridge-type (CBT) displacement amplifier for piezoelectric drives. The proposed CBT model was describe of having a large AR and lateral stiffness which makes it more suitable for actuator protection and isolation than the ordinary bridge-type amplifiers. The proposed CBT design has been optimized for a large resonance frequency subject to other performance constraints. Since the developed CBT model was capable of generation an output displacement over $1[\mathrm{~mm}]$, it was deemed suitable for development of micro/nano positioning stages with a cubic millimeter sized workspace. The proposed CBT mechanism employed right-circular flexure hinges, however; for this model also it was suggested that other hinge geometries can be implemented.

Park and Yang (2005) provided detailed procedure for the design and analysis of a 6-DOF ultra precision positioning system. Their mechanism employed monolithic and symmetric structure to reduce compliant errors of the flexure hinges caused by mechanical noise and thermal effect. To obtain high positioning accuracy symmetric circular flexure hinge were incorporated in their design. To achieve 6-DOF an inclined bridge displacement amplifier 
was adopted. The proposed mechanism also features an amplifying mechanism to achieve longer stroke than the one provided by the PZT actuator.

Palli et al. (2011) proposed a novel force sensor based on commercial discrete optoelectronic components mounted on a compliant frame. The compliant frame of the mechanism has been optimized to achieve the desired relation between the applied force and the angular displacement of the optical axes of the optoelectronic components. To achieve a symmetric structure, the compliant frame has been designed in the form of tow identical slider-crank mechanisms attached to each other from the vertical line of symmetry. Corner filleted flexure hinges have been employed for the connection between the linkages of the compliant frame. The proposed sensor mechanism was deemed suitable for applications in the field of tendon driven robots.

For nano -positioning purposes $\mathrm{Xu}$ (2012) designed and developed a new flexure-based dual stage system which features a new decoupling design to minimize the interference behavior between the coarse motion generator and the fine motion generator stages of the device. The output displacement of the proposed mechanism is guided by a compliant mechanism which is composed of eight right-circular hinges.

Polit et al. (2011) also presented a high-bandwidth (i.e. NF) XY nano-positioning system. The developed system was designed to achieve a high NF with minimizing the parasitic motion. The structure of the device was based on the parallel kinematic mechanism, featuring two parallel independent kinematic chains. Each kinematic chain consisted of a prismatic joint connected to PZT actuator and a parallelogram hybrid flexure mechanism connected to the end-effector of the device. Kinematic and dynamic analysis of the mechanism revealed 
that the compliant structure of the stage possessed a high NF and decoupled motion in XY directions. The proposed mechanism can be employed the applications in high-throughput nano-scale metrology, imaging, and manufacturing.

Bharti et al. (2003) described a methodology of designing compliant mechanisms with PZT actuation to obtain maximized deflection and force at output. These objectives were achieved by designing compliant mechanisms with multiple optimally placed and sized PZT actuators in the body of the compliant mechanism. In another word the introduced method optimizes the number of PZT actuators employed, their sizes, and their effective locations within the body of the compliant mechanism in order to maximize the AR of the device against an external spring. Results of their study proved that the proposed compliant mechanism topology based on the multiple, optimally placed PZT actuators methodology out-performs the one with a single PZT actuator placed at a predetermined location.

The following table is the list of existed compliant mechanical amplifiers discussed in this section:

Table 2.1: Existed compliant mechanical amplifiers

$\begin{array}{ll}\text { Compliant Mechanical Amplifier } & \text { Developer(s) }\end{array}$ mechanisms 


\subsection{Environmental Consideration in the Design of Compliant Mechanisms}

Some applications of micro manipulator stages require operation at variable temperature environments. Same as mechanical forces, temperature variations can also cause deformations and stresses within the structure of the compliant mechanism (Przemieniecki, 1976). Thermal effects due to temperature variations can dramatically alter the performance of the precision positioning stage of compliant based micro manipulator systems. Consequently, it is essential to consider the thermal effects in the analysis and design of compliant mechanism systems if they are supposed to be operated in variable temperature environments (Zhang \& Hou, 2010). 
Numerous studies have been conducted to investigate the impacts of thermal effects on the static and dynamic performances of compliant mechanisms. For example, Manoach et al. (2004) studied geometrical nonlinear vibrations of the moderately thick beams under the combined action of mechanical and thermal loads. The results of their study suggested that short heat pulses with high magnitudes might cause vibrations with considerably large amplitudes which may have some undesirable effects on the performance of the device.

In another study Hou and Zhang (2010) investigated the dynamic performances of the flexible linkage mechanisms under uniform temperature change. Their numerical solution analysis indicated that a minute change in temperature will cause a significant change of the stresses experienced by a flexible mechanism.

Hua et al. (2008) also presented the dynamic analysis and experimental results for a 3-DOF flexure-hinge-based precision positioning stage. To consider the thermal effects they treated the right-circular flexure hinge as a three nodes element, in which there are three degrees of freedom at each node, and the center offset of the hinge was evaluated directly. The results of their analysis indicated that the steady-state response of stresses of the stage were decreasing or increased almost linearly with the increasing of the input displacement at the same temperature. They have also observed that, for the same input displacement, the stresses experienced by the mechanism were increased with increasing the temperature; and finally the more the temperature rise or decrease, the more significant the stress is changed.

\subsection{Topology Optimization Techniques}

After selecting the best topology for the design of the compliant mechanism, topology

optimization of the proposed design should be performed in order to obtain the best 
performing mechanism for achieving a particular task(s). This section is the review of different optimization techniques used to optimize the kinematic and dynamic performance of the proposed design of compliant mechanisms.

Dinesh et al. (2007) proposed a structural topology optimization of a parallel arranged largerange compliant $\mathrm{XY}$ micro stage mechanism as illustrated in Figure 2.7. The objective of their study was to maximize mobility of the motion stage of the proposed compliant mechanism while decoupling the motion of the stage due to applied inputs in two orthogonal directions. Consequently, the proposed optimization technique was described as a multi objective optimization with the following two functions:

$$
\begin{aligned}
& f_{1}=-2 \frac{v_{2, \text { stage }}}{v_{2, \text { in }}} \\
& f_{2}=2 \sqrt{\left(\frac{u_{2, \text { stage }}}{v_{2, \text { in }}}\right)^{2}}
\end{aligned}
$$

where $v_{2, i n}$ is the input displacement created by $F_{y}$ at the mechanism's input at $E$, while $v_{2, \text { stage }}$ and $u_{2, \text { stage }}$ are the output displacements in $\mathrm{Y}$ and $\mathrm{X}$ directions respectively as shown in. It was also noted that the objective function $f_{l}$ maximizes the amplification in both $\mathrm{X}$ and $\mathrm{Y}$ directions whereas the objective function $f_{2}$ minimized the coupling effect of the orthogonal inputs at $D$ and $E$. These two objectives are obtained by minimizing the widths (w) of all compliant frames that the mechanism is consisted of. As shown in Figure 2.7, the diagonal symmetry of the structure causes the optimization in one direction takes effect in both directions. Hence, instead of the input displacement generated by $F_{y}$ and the consequent output displacements, the created input and the eventual output displacements generated by $F_{x}$ can be used in the two defined objective functions. 


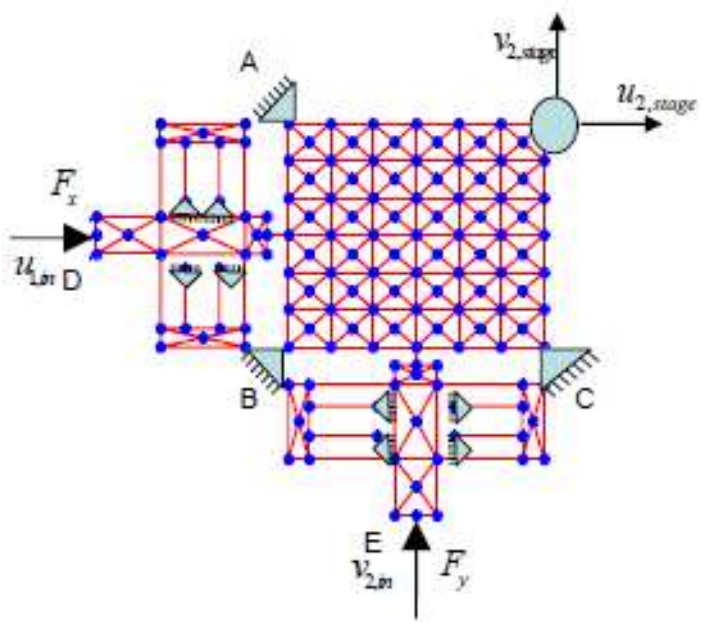

Figure 2.7 The ground structure of frame members (Dinesh \& Ananthasuresh, December 12-13, 2007).

Finally a weighted sum method (Marler \& Arora, 2006) was suggested to tackle the above multi objective problem as the following:

$$
F=\lambda f_{1}(w)+(1-\lambda) f_{2}(w)
$$

where the weighting parameter $\lambda$ was varied parametrically between 0 and 1 to obtain the best performing device based on the combination of the two objective functions.

Shih and Chen (2008) introduced an optimum topological design for a compliant mechanical amplifier integrated with a PZT nano-positioning stage. The proposed design has been described as one-piece compound compliant mechanism which comprised of an amplifier and nano-motion bed (i.e. output). The proposed mechanism was then optimized for a high stiffness resulting into a NF more than $200[\mathrm{~Hz}]$, and a maximum output displacement. Consequently the optimization of the mechanism was achieved by treating it as a multiobjective optimization problem. In another word, the output displacement of the device has been maximized by constraining the density of the material within a certain limit in order to achieve the required stiffness for the target NF. 
In another study, Shih et al. (2006) proposes a two stage optimization technique to find the optimum flexure configuration and location of flexure hinges to increase the overall performance of a compliant micro gripper. The focus of the first stage of the optimization process was to maximize the work performed on the spring characteristics of the griper's flexure hinges by distributing a limited amount of material in the design domain such that the output displacement was maximized. In another word, the first stage optimization was used to find the size of the required flexure hinges to maximize the performance of the micro gripper. The proposed second stage optimization was used to find the optimal location of the flexure hinges found in the first stage optimization. The second stage optimization was achieved by ensuring that the maximum allowable stress of the used material has not been breached.

Pedersen and Seshia (2004) presented the optimization of a compliant force amplifier mechanism in a surface micro-machined resonant accelerometer. Figure 2.8 illustrates the schematic of half of the proposed resonant accelerometer where $\Omega$ is the assumed design domain. Due to symmetry of the proposed structure only half of the resonant accelerometer was analysed.

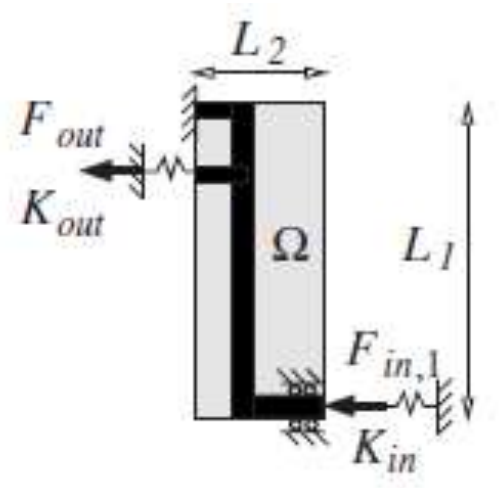

Figure 2.8 half of the resonant accelerometer with symmetry supports applied (Pedersen \& Seshia, 2004) 
Their introduced optimization technique has been constrained by limitations imposed by the manufacturing process and the device geometry. In the presented study it was discovered that the topology optimization of the device depended dramatically on the size of the design space, output and input stiffnesses and imposed boundary conditions. In order to max the output force of the mechanism, they have defined the following objective function:

$$
\max (A)=\max \left(\frac{F_{\text {out }}}{F_{\text {in }}}\right)=\max \left(\frac{k_{\text {out }} u_{\text {out }}}{F_{\text {in }}}\right)=\frac{k_{\text {out }}}{F_{\text {in }}} \max u_{\text {out }} \quad 2.4
$$

where $A$ corresponds to maximum force amplification and $k_{\text {out }}$ is the output stiffness of the device. As it can be implied form Equation 4 in order for the device to have the maximum force amplification, the output displacement (i.e. $u_{\text {out }}$ ) of the mechanism had to be maximized.

\subsection{Topology of Symmetric Five-Bar Structure}

The design and optimization of the two proposed CMDA mechanisms in this thesis are based on the symmetric five-bar structure developed by Ouyang et al. (2008). As mentioned in previous chapter, the symmetric five-bar topology facilities many advantages over other presented most common topologies used for displacement amplification purposes. In this research thesis it will be shown that with appropriated modifications, the symmetric-five bar topology can be made compatible for achieving a particular task for a given application.

This chapter presents the general characteristics and pseudo-rigid body model (PRBM) formulation of the symmetric five-bar topology used as the design basis for the development and optimization of the Novel High Amplitude Piezoceramic Actuator for Applications in 
Magnetic Resonance Elastography and the XY Compliant Mechanical Displacement Amplifier.

\subsubsection{Design and PRBM Analysis of the symmetric Five-Bar Structure}

The symmetric five-bar structure is symmetrical in its configuration. As shown in Figure 2.9 a PZT actuator is used to produce the input displacement $\delta$ required for simultaneous rotations of two driving links in opposite directions. This then generates an output displacement of $y$ that can be constrained in one direction only. As mentioned earlier this topology is actually a combination of a symmetric four bar topology and a lever arm topology. In another word the vertical bars are of lever arm structures, and between the two lever arms is a symmetric four-bar structure, see Figure 2.9. The advantage of this configuration over the symmetric four-bar topology is its high NF and AR in a compact size.

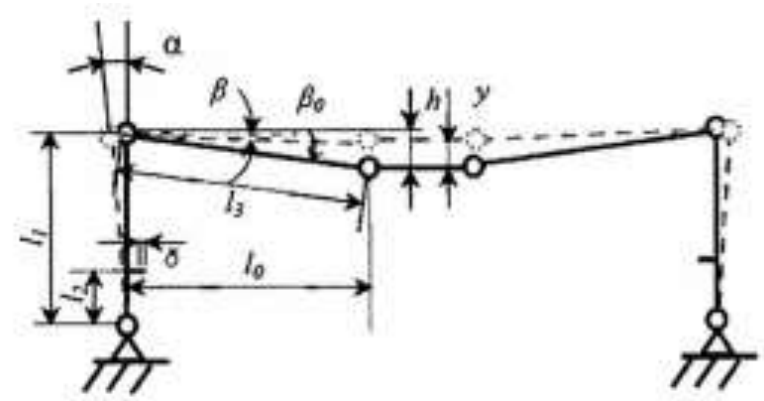

Figure 2.9 The pseudo rigid body model of the symmetric five-bar topology (Ouyang, Zhang, \& Gupta, 2008)

Figure 2.9 also shows a PRBM of the symmetric five-bar topology. The PRBM model can be used to predict the output displacement $y$ and overall stiffness $K$ of the CMDA based on this topology as the following (Ouyang, Zhang, \& Gupta):

$$
\sin \alpha=\frac{\delta}{l_{2}}
$$




$$
\begin{gathered}
\cos \beta=\frac{l_{1} \sin \alpha+l_{0}}{l_{3}} \\
y=h-l_{3} \sin \beta-l_{1}+l_{1} \cos \alpha
\end{gathered}
$$

where $\alpha$ is the relative rotation of the input link, caused by $\delta, h$ is the initial high of the uniformed structure, and $l_{i}$ is the length of the link $l$. Since the input $\delta$ is of the order of micrometers $[\mu \mathrm{m}]$ and structure's links $l_{i}$ are of the order of centimeters $[\mathrm{cm}]$, then the angle $\alpha$ will be in order of microrads. Consequently, if we let $k$ represent the input amplification of the PZT actuator by the lever arm, expressed as $k=\frac{l_{1}}{l_{2}}$. , Equations 2.6 and 2.7 can be written as:

$$
\begin{aligned}
& l_{3} \sin \beta=h-y \\
& l_{3} \cos \beta=l_{0}+k \delta
\end{aligned}
$$

Form Figure 3.1 we have deduced that $h^{2}+l_{0}^{2}=l_{3}^{2}$. Therefore, from Equations 2.8 and 2.9 we can obtain:

$$
y^{2}-2 h y+\left(2 l_{0}+k \delta\right) k \delta=0
$$

By solving equation 2.10 the output displacement $y$ can then be found as the following:

$$
y=h-\sqrt{h^{2}-\left(k \delta+2 l_{0}\right) k \delta}
$$

From the PRBM in Figure 2.9 and the above formulation, it can be concluded that the AR is directly related to the ratio of $l_{1} / l_{2}, h$, and $l_{0}$. Also it is certain that the profile of the flexible hinge has significant contribution to the AR. 
The stiffness $K$ of the corner-filleted flexure hinge used in the structure of a CMA based on the symmetric five bar topology, has significant effects both on the displacement amplification and the dynamic characteristics of the mechanism. Using formulation developed by Lobontiu et al. (2001) $K$ can be found as the following:

$$
K=\frac{E b t^{3}}{12(l-2 r f(\gamma))}
$$

where $E$ is the modulus of elasticity of the material, $b$ and $t$ of the width and thickness of the flexure hinge, $l$ is the length of the corner filleted flexure hinge, and $r$ is the fillet radius. Please note that in above equation $\gamma=\frac{2 r}{t}$ and

$$
f(\gamma)=\frac{(2 \gamma+1)\left(3 \gamma^{2}+2 \gamma+1\right)+3 \gamma(\gamma+1)^{2} \sqrt{2 \gamma+1} \tan ^{-1}(\sqrt{2 \gamma+1)}}{(\gamma+1)(2 \gamma+1)^{3}}
$$

From Equation 2.12 it can be observed that the parameter that affects the stiffness of a flexure hinge most significantly is the thickness $t$. The stiffness increases exponentially with the increase in $t$. On the other hand, stiffness is proportional to the width $b$ of the flexure hinge. In addition, the length $l$ of the corner-filleted hinge has some inverse influence on the stiffness (Ouyang, Zhang, \& Gupta, 2008).

\subsection{Concluding Remarks}

This chapter presented a review on the most common topologies of compliant mechanisms available for mainly displacement amplification purposes. It was also discussed that due to advantages facilitated flexure hinges of compliant structures, an amplifier based on the compliant mechanism is best option to increase the output displacement of a PZT actuator. 
The thermal effects on the performance of the compliant mechanism were also discussed on this chapter. Several papers, detailing the effects of temperature variation on the kinematic and dynamic performance of compliant mechanism as well as the dramatic changes on the stress distribution across the flexure hinges were presented.

Finally, it was shown that topological optimization is required to achieve the optimal performance of a designed compliant mechanism for various purposes such as force amplification, displacement amplification, and increasing the systems overall stiffness.

The rest of this thesis refers to two developed CMDA mechanisms based on the five bar symmetric topology. The objectives, design process, topological optimization, FEM analysis results of each mechanism are also discussed in the following chapters. 


\section{A NOVEL HIGH AMPLITUDE PIEZOCERAMIC ACTUATOR FOR APPLICATIONS IN MAGNETIC RESONANCE ELASTOGRAPHY}

The design and development of the novel high amplitude PZT actuator for application in MRE was a joint project between Ryerson University Department of Aerospace Engineering and University of Toronto Department of Bio-Medical Physics. As shown in Figure 3.1 Arani et al. (2011) developed a device which uses a rigid endorectal coil coupled directly mechanically to a PZT actuator to generate shear waves in the prostate gland. The developed device incorporates prostate stiffness characterization into multiparametric magnetic resonance imaging since prostate cancer causes some changes in the elasticity of prostate tissue.

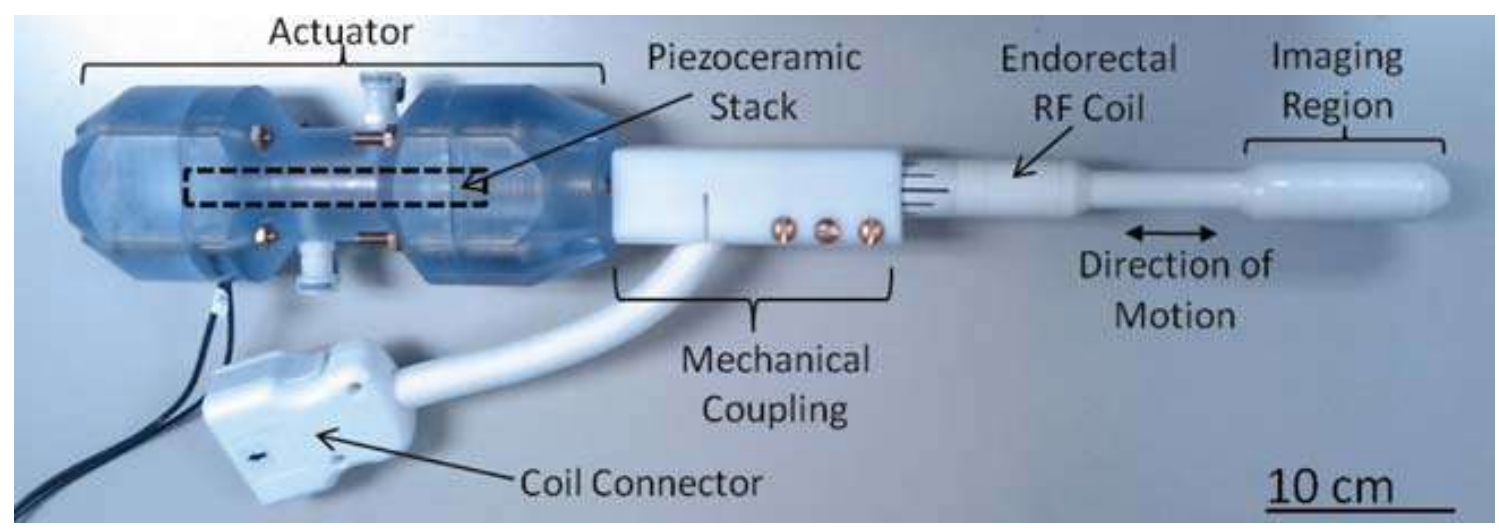

Figure 3.1 A Photo of the mechanically coupled piezoceramic actuator ( (Arani, Plewes, Krieger, \& Chopra, 2011)

The performance of the developed apparatus however, can be significantly improved by integrating the PZT actuator with a CMDA and coupling it to the rigid endorectal coil. The CMDA amplifies the amplitude of the waves generated by the PZT and helps the waves to travel farther into the prostate. This will result in better MRI imaging and identifying the exact location of the suspicious cancerous tissues in prostate. 
This chapter provides the detailed FEM and experimental analysis of the designed CMDA for the used in the the novel high amplitude PZT actuator for application in MRE. The optimization process and technique to achieve the best performing CMDA according to the given requirements are also discussed in this chapter.

\subsection{Design Requirements and constraints}

The goal of this research is to exploit the advantages of the symmetric five-bar topology to propose a CMDA capable of amplifying the displacement generated by the PZT actuator attached to it, with high NF.

Figure 3.2 shows the FEM model of the proposed CMDA for the use in prostate MRE application. As illustrated in the figure, the CMDA is symmetric along the vertical axis and it is constraint to translate the horizontal driving force and motion of the PZT to the Y direction motion at its output. Please note that the base of the Proposed CMDA is also constrained in all degree of freedoms.

According to Figure 3.2 the proposed amplifier has the following characteristics:

1) The mechanism is a symmetric compliant mechanism with one degree of freedoms (DOF).

2) The applied CMDA amplifies the stroke of the PZT actuator.

3) The designed mechanism can be used to generate linear micro motions only.

4) The moving output or end-effector of the device is design to accommodate the rigid endorectal coil.

5) The proposed CMDA design consists of two pairs of the inclined bars of the length $l_{3}$ (Figure 3.3) to increase the NF of the designed mechanism. 


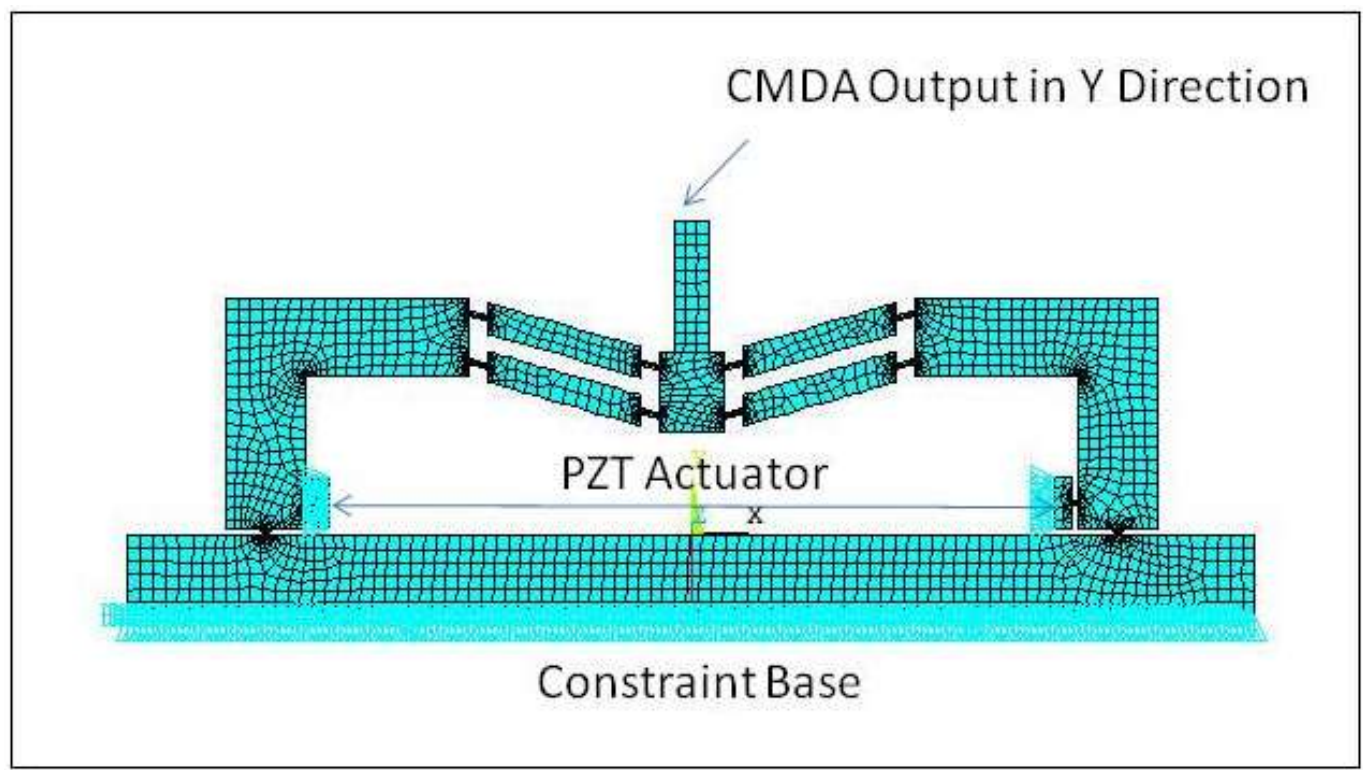

Figure 3.2 FEM model of the proposed CMDA for the use in prostate MRE application.

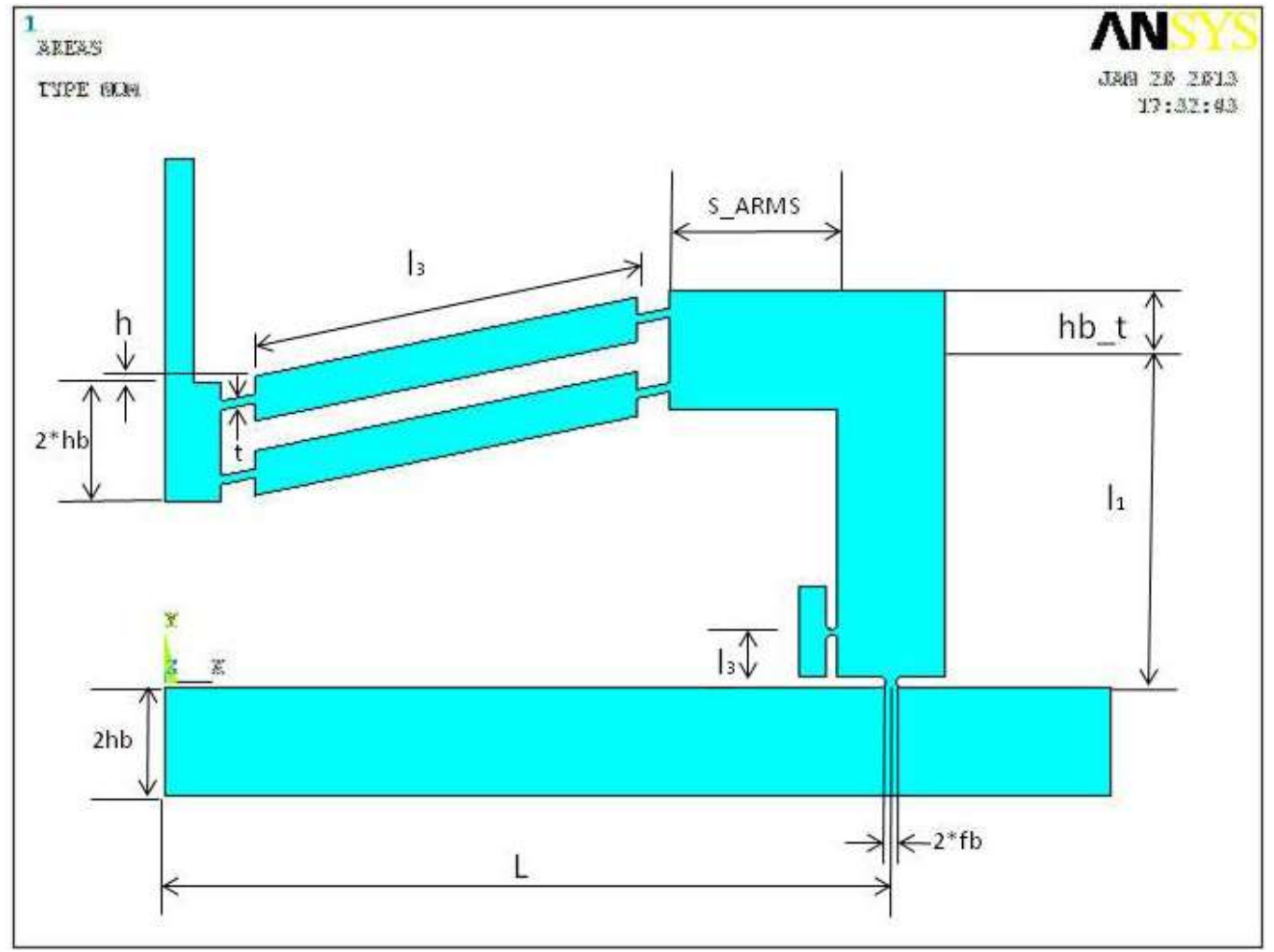

Figure 3.3 Key parameters used for the design of the CMDA for application in prostate MRE. 
Figure 3.3 shows some of the key design parameters that have the most significant effect on the performance of the CMDA. Please note that due to the symmetric structure of the device these parameters are shown only on half of the designed structure.

Table 3.1 below also lists the initial values of the most important parameters used to design the initial model of the proposed CMDA. Please note that the minimum dimension that parameter $L$ can adopt is related to the length of the PICA ${ }^{\mathrm{TM}} P-010.80 P$ PZT actuator that will be integrated within the amplifier. Parameter $h$ also stipulates the inclined angle of the double parallel bars, referring to Figure 2.9. $S \_A R M S$ and $h b \_t$ are the length and the width of the horizontal section of the lever arm component of the mechanism. The width of the mechanism's base is a function of the parameter $h b$. Finally parameters $t$ and $f b$ represent the thickness of the rectangular and circular flexure hinges, respectively. The thickness of the CMDA is $12.7 \mathrm{~mm}$.

Table 3.1: Design parameters of the proposed CMDA for the use in prostate MRE application (unit: $\mathbf{m m}$ )

\begin{tabular}{ccccccccccc}
\hline Parameters & $L$ & \multicolumn{1}{c}{$l_{l}$} & $S_{-}$ARMS & $t$ & $l_{2}$ & $h$ & $f b$ & $l_{3}$ & $\boldsymbol{h b}$ & $\boldsymbol{h b} \boldsymbol{t}$ \\
\hline \multirow{2}{*}{ Dimension } & 65 & 29.753 & 25 & 0.8 & 4.5 & 8.318 & 0.5 & 23.9 & 6.0 & 6.0 \\
\hline
\end{tabular}

\subsection{Initial FEM Static and Dynamic Analysis}

A 2-D model of the proposed design of the CMDA for the use in prostate MRE application was constructed using ANSYS ${ }^{\circledR}$ Mechanical APDL with the primary parameters listed in Table 3.1. Then by assigning the brass material properties (Table 3.2) to the model, the CMDA was meshed using PLANE 82 element type. PLANE 82 is a higher order 2-D 8-node plane element with each node having two translational degrees of freedom in $X$ and $Y$ nodal 
directions (ANSYS, 2009). This element supports plasticity, creep, swelling, stress stiffening, large deflection, and large strain.

Table 3.2: Brass material properties

\begin{tabular}{lc}
\hline Modulus of Elasticity [GPa] & 97.2 \\
Density $\left[\mathrm{kg} / \mathrm{m}^{3}\right]$ & 8934 \\
Poisson's Ratio & 0.311 \\
& \\
Yield Strength $[\mathrm{MPa}]$ & 359 \\
\hline
\end{tabular}

After completing the above procedure the initial static and dynamic FEM analyses to obtain the AR and NF of the proposed CMDA was performed by applying input displacements of $10[\mu \mathrm{m}]$ to each of the device's two inputs (the contact location between the device input and the end effects of the PZT actuator) to simulate the displacement generated by PZT actuator. Please note that the AR is the ratio of the maximum output displacement $(y)$ of the device to the input displacement $(\delta)$ created by PZT actuator:

$$
A R=y / \delta
$$

The results obtained from the above analysis are listed in Table 3.3.

Table 3.3: Initial Dynamic and Static Analysis Results

\begin{tabular}{ccc}
\hline $\mathbf{N F}[\mathrm{Hz}]$ & $\mathbf{A R}$ & Max. Stress $[\mathbf{M P a}]$ \\
\hline 475.54 & 14.7 & 141 \\
\hline
\end{tabular}

As it can be seen from Table 3 and Figure 3.4, the mechanism has an AR of 14.7 and a NF of $475.54[\mathrm{~Hz}]$. Figure 3.4 also illustrates that the maximum displacement of $147[\mu \mathrm{m}]$ occurs at 
the device's output when a displacement of $10[\mu \mathrm{m}]$ applied to each of the two CMDA inputs.

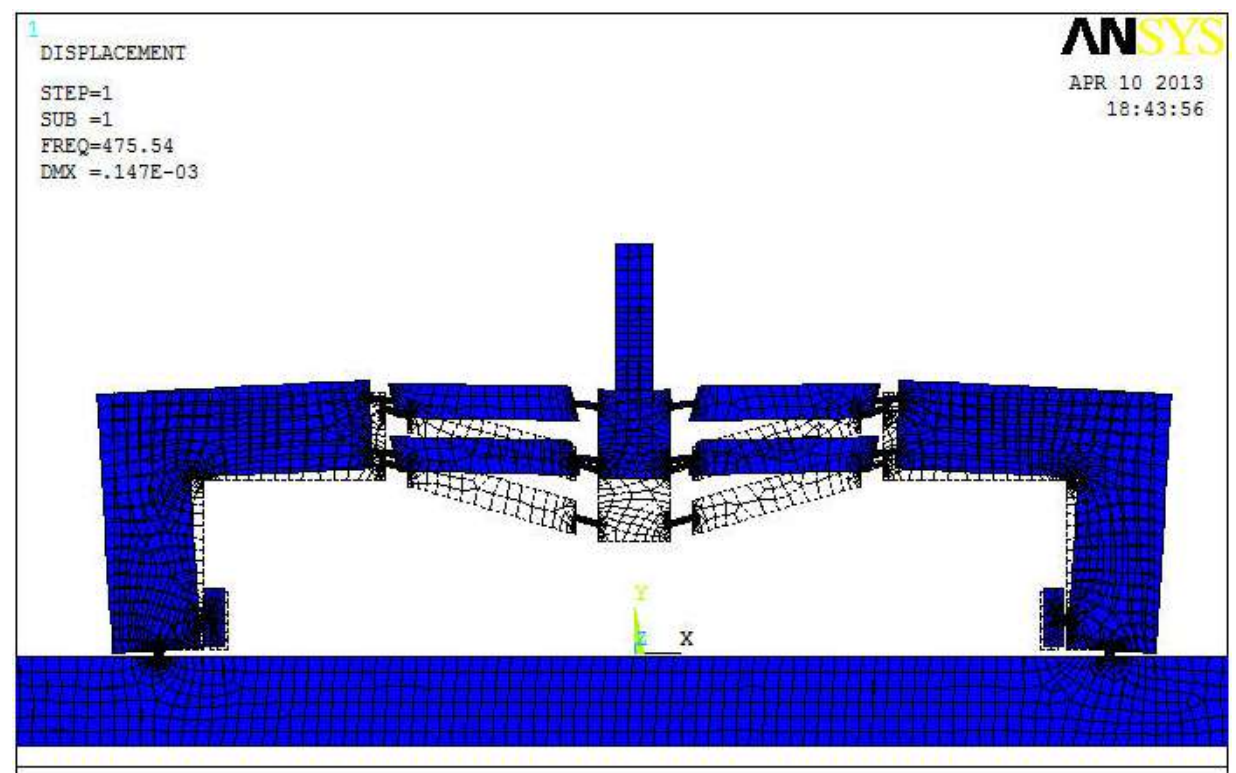

Figure 3.4 Deformed shape of the initial CMDA design when $10[\mu m]$ input displacement is applied.

Figure 3.5 is the contour plot of the Von-Mises stress of the CMDA when displacements of $10[\mu \mathrm{m}]$ were applied to its inputs. Figure 3.5 illustrates that the CMDA experiences a maximum stress of $141[\mathrm{MPa}]$ at the left circular flexure hinge attaching the lever arm component of the mechanism to its base. A close up image of the mentioned high stress are is shown if Figure 3.6. This high stress area is marked on the figure by $M X$. Certainly, the maximum stress is much less than the Yield Strength of the material 


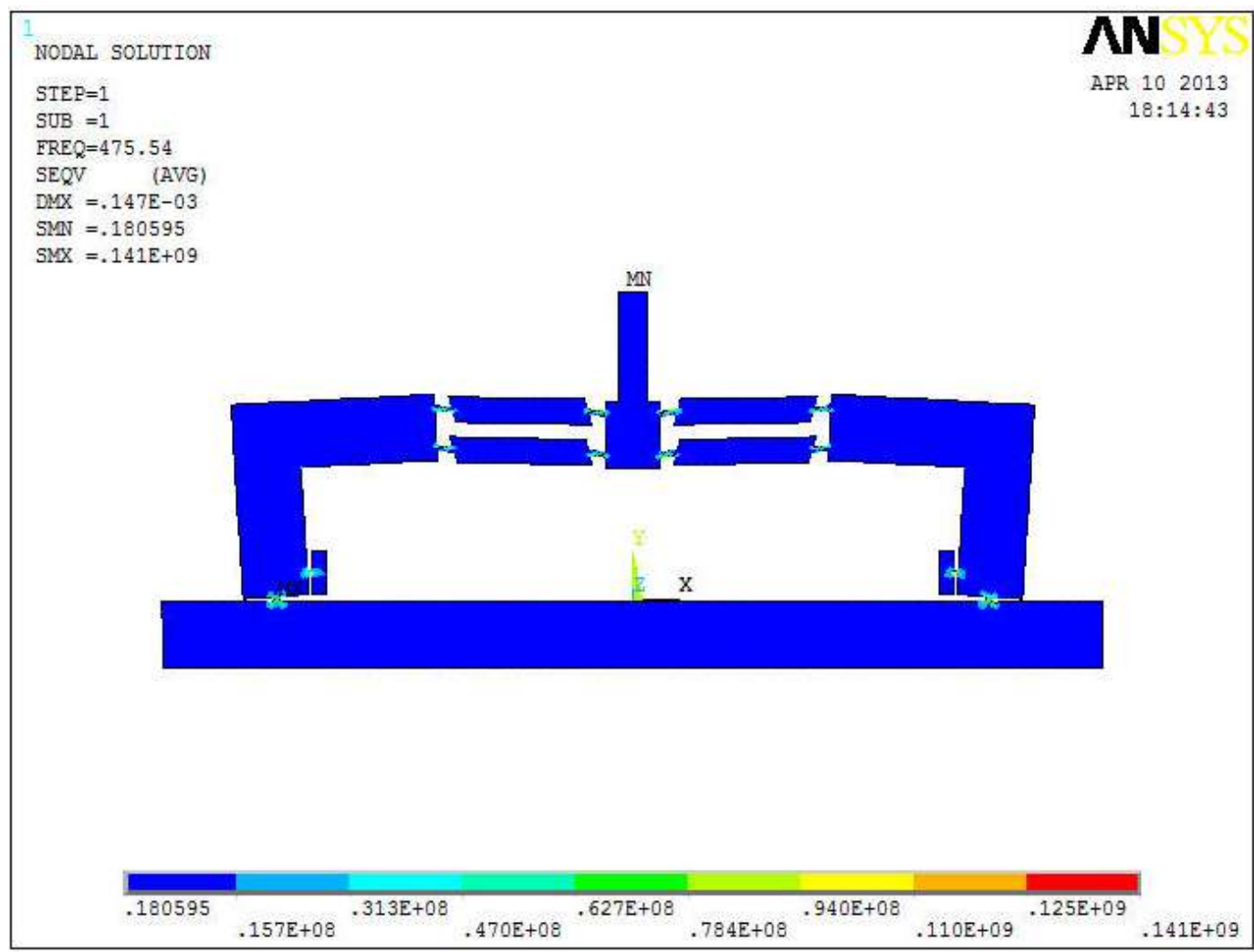

Figure 3.5. Contour plot of the Von-Mises stress of the initial CMDA.

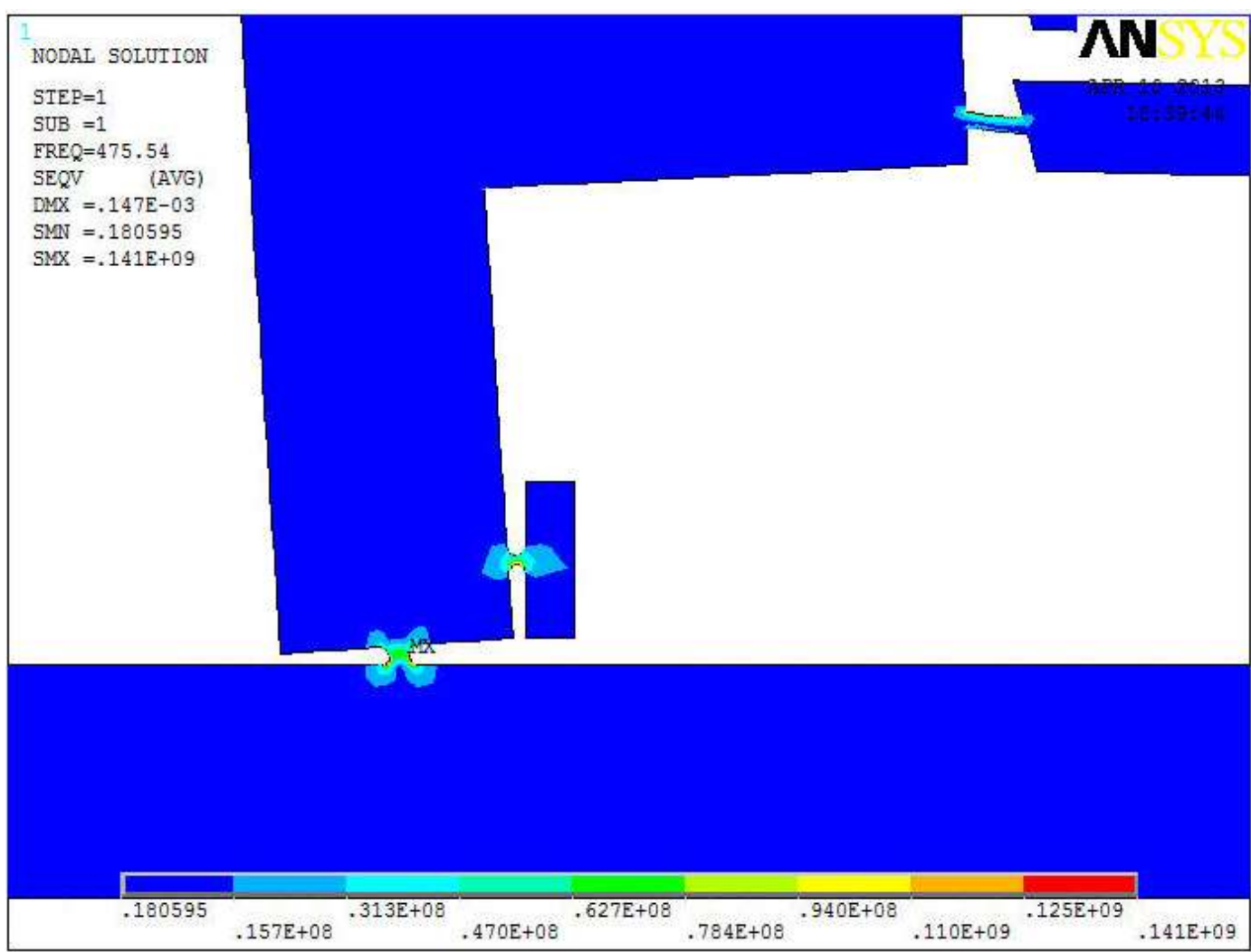

Figure 3.6 Contour plot of the Von-Mises stress of the left circular flexure hinge of the initial CMDA. 


\subsection{Optimal Design}

\subsubsection{Design Parameters}

The goal of an optimization process for the proposed CMDA design for application in prostate MRE is to obtain the values of some significant design parameters that maximize the AR of the device with taking into account the maximum stress occurred in the flexural hinges and the maximum force available by PZT actuators to create a desired input displacement. These significant parameters were regarded as having the most effects on the AR of the symmetric five-bar CMDA with having minimal effect on the dynamic performance (i.e. NF) of the mechanism. Table 3.4 lists the design parameters used for the optimization of the proposed CMDA with their corresponding allowable ranges. It should be noted that selected ranges of the design parameters are based on some sub-optimal design results.

Table 3.4: Design Parameters and their range used for optimization (unit:
\begin{tabular}{cc}
\hline Design Parameter & Range \\
\hline$l_{l}$ & $29.753-45.00$ \\
$t$ & $0.80-1.40$ \\
$h b_{-} t$ & $4.00-8.00$ \\
$h$ & $8.318-8.90$ \\
$h b$ & $4.00-8.00$ \\
S_ARMS & $15.00-45.00$ \\
\hline
\end{tabular}

\subsubsection{Optimization Process}

As mentioned earlier, one of the optimization constraints is the maximum stress that flexural hinges can undergo. This was defined by brass yield strength listed in Table 2. In another word during optimization process, the maximum stress $(\sigma)$ experienced within flexural hinges 
was not allowed to exceed brass yield strength $\left(\sigma_{y}\right)$ with a safety factor $(\mathrm{SF})$ which was chosen to be one. Another constraint considered for the optimization was the force required to create a given amount of input displacement. This force also was set to not exceed $P I C A^{\mathrm{TM}}$ $P-010.80 P$ PZT actuator push load capacity of $2300[N]$ during the optimization process.

Therefore, the optimization problem of the proposed CMDA can be described as:

$$
\begin{gathered}
\text { Maximum: } A R=y / \delta \\
\text { Subject to: } \\
\sigma<\sigma_{y} / S F \\
F+(19 E 6) *(\delta)<2300
\end{gathered}
$$

where $F$ is the force required to create a displacement at each of the device's input, and the constant 19E6 in Equation 3.3 is the actuator inherent stiffness.

The optimization problem of the proposed CMDA design can however, be described as a multi-objective one since the goal of the optimization is to maximize the AR with minimizing the consequent drop in NF. To achieve the mentioned goal the following objective function $(\mathrm{Obj})$ is defined:

$$
O B J=100-\left[\frac{w_{1} * D M A X}{140 E-6}+\frac{w_{2} * F R E Q 1}{500}\right]
$$

where $w_{1}$ and $w_{2}$ are the weighting factors each varying between 0.1 and $0.9, D M A X$ and FREQ1 are the output displacement and NF obtained for a set of design parameters, respectively. Finally $140 E-6$ and 500 are the target values of the output displacement and NF respectively. In order to obtain the optimized values of design parameters the overall 
value of the OBJ function has to be minimized. In another word, the parameters corresponding to the OBJ function with the smallest value are the ones which yield the best performing CMDA with the combination of high AR and NF.

As there is no explicit formula to build the connections between the design parameters and the objective function and constraints, a numerical optimization analysis using ANSYS is approached. ANSYS first order optimization method with 30 iterations per trial was used to find the design parameters (within the given range) that yields the best AR for the proposed design for a constant $10[\mu \mathrm{m}]$ input displacement, while two constraints are satisfied at the same time. Please note that the first set of optimized results was obtained by assigning $w_{1}=0.9$ and $w_{2}=0.1$ to the objective function. The next sets of optimizations results were then achieved by decrementing $w_{1}$ by 0.1 and incrementing $w_{2}$ by 0.1 until $w_{1}$ reached the value of 0.1 and $w_{2}$ reached the value of 0.9 . The optimized parameters under this specific condition are obtained through ANSYS and listed in Appendix A.

Table 3.5 also lists the design parameters obtained for the best performing CMDA within an acceptable combination of high AR and NR. These values were achieved by using the OBJ function with the weighting factors of $w_{1}=0.8$ and $w_{2}=0.2$.

Table 3.5: Design Parameters obtained after optimization (unit: $\mathbf{m m}$ )

\begin{tabular}{cc}
\hline Design Parameter & Range \\
\hline$l_{l}$ & 29.753 \\
$t$ & 0.80 \\
$h b \_t$ & 5.3289 \\
$h$ & 8.318 \\
$h b$ & 4.8794 \\
$S \_A R M S$ & 15.00 \\
\hline
\end{tabular}


Figure 3.7 is the plot of the design parameters versus the design objective (OBJ function). As it can be seen from the graph the listed optimized design parameters in Table 3.5 to OBJ function of 99.059. Form this figure it can also be implied that $h b$ and $h b \_t$, can moderately affect the AR and NF of the device. On the other hand, the minimum set values of parameter $S \_A R M S$ and $t$ (Table 3.4) proved to yield best AR and NR while parameters $h$ and $l_{l}$ maintained relatively constant values throughout the optimization process. Please note that smaller value of the thickness $t$ yield a higher AR. However, thinner flexure hinges can sustain less stress for a given input displacement and will result in the CMDA failure.

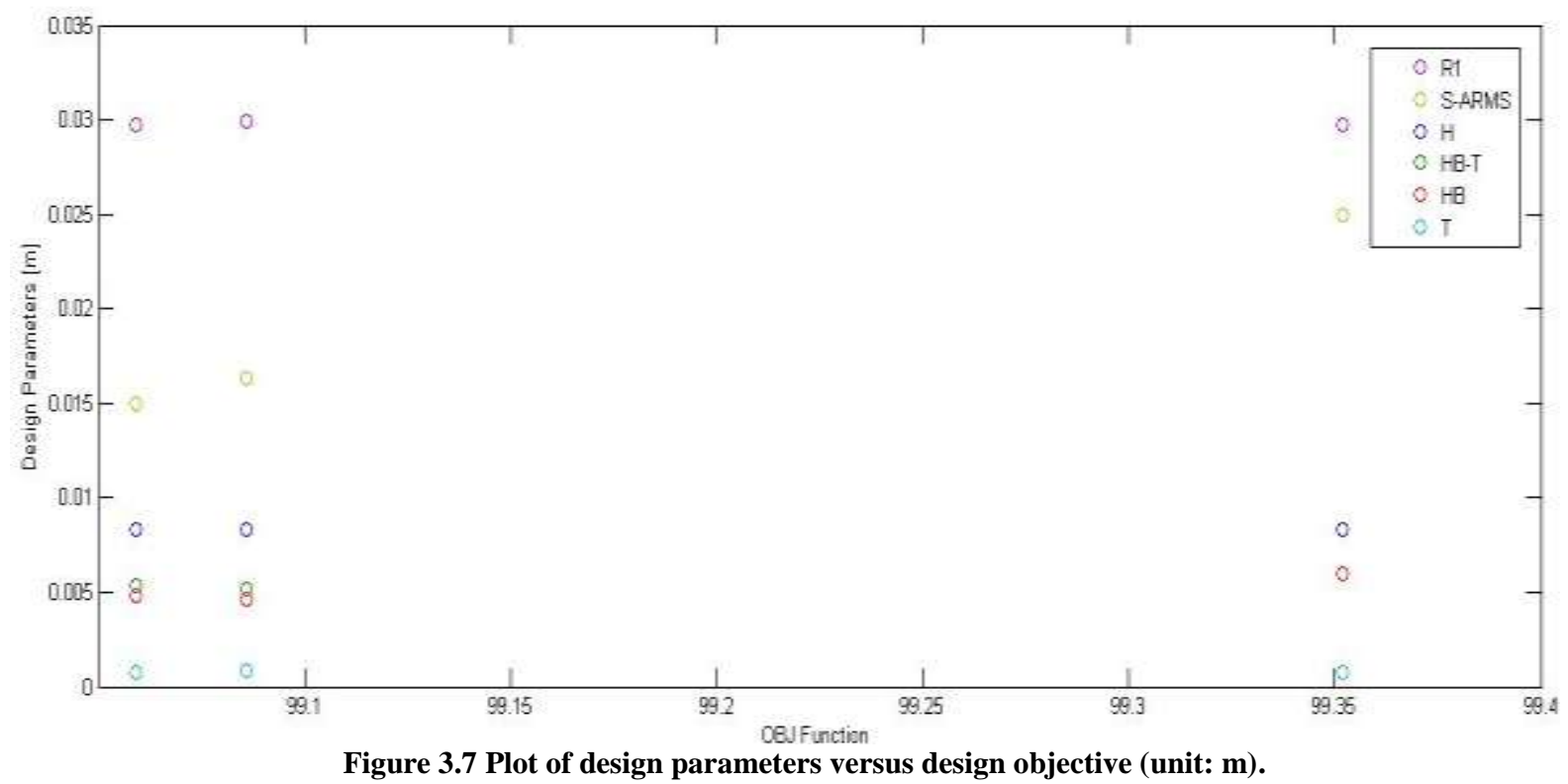

\subsubsection{Static and Dynamic FEM Analysis on the Optimized Design (Unloaded CMDA)}

After obtaining the optimized design parameters, static and dynamic FEM analysis were performed on the proposed optimized unloaded CMDA. Table 3.6 lists the dynamic and static results of the proposed optimized design. 
Table 3.6: Optimized Dynamic and Static Analysis Results

\begin{tabular}{ccc}
\hline NF $[\mathbf{H z}]$ & AR & Max. Stress $[\mathbf{M P a}]$ \\
\hline 384.071 & 19.2 & 135 \\
\hline
\end{tabular}

Figure 3.8 illustrates the contour plot of the Von-Mises stress of the optimized CMDA when displacements of $10[\mu \mathrm{m}]$ were applied to both of its inputs. From this figure, it can be observed that the location of the maximum stress experienced by the CMDA has changed to the location of the circular flexure hinge attaching the left input to the vertical component of the lever arm portion of the device. This high stress area is labelled as $M X$ on the Figure 3.9 as well. The maximum stress experienced by the CMA structure is $135[\mathrm{MPa}]$ which shows a decrees of $6[\mathrm{MPa}]$ in comparison to the un-optimized case.

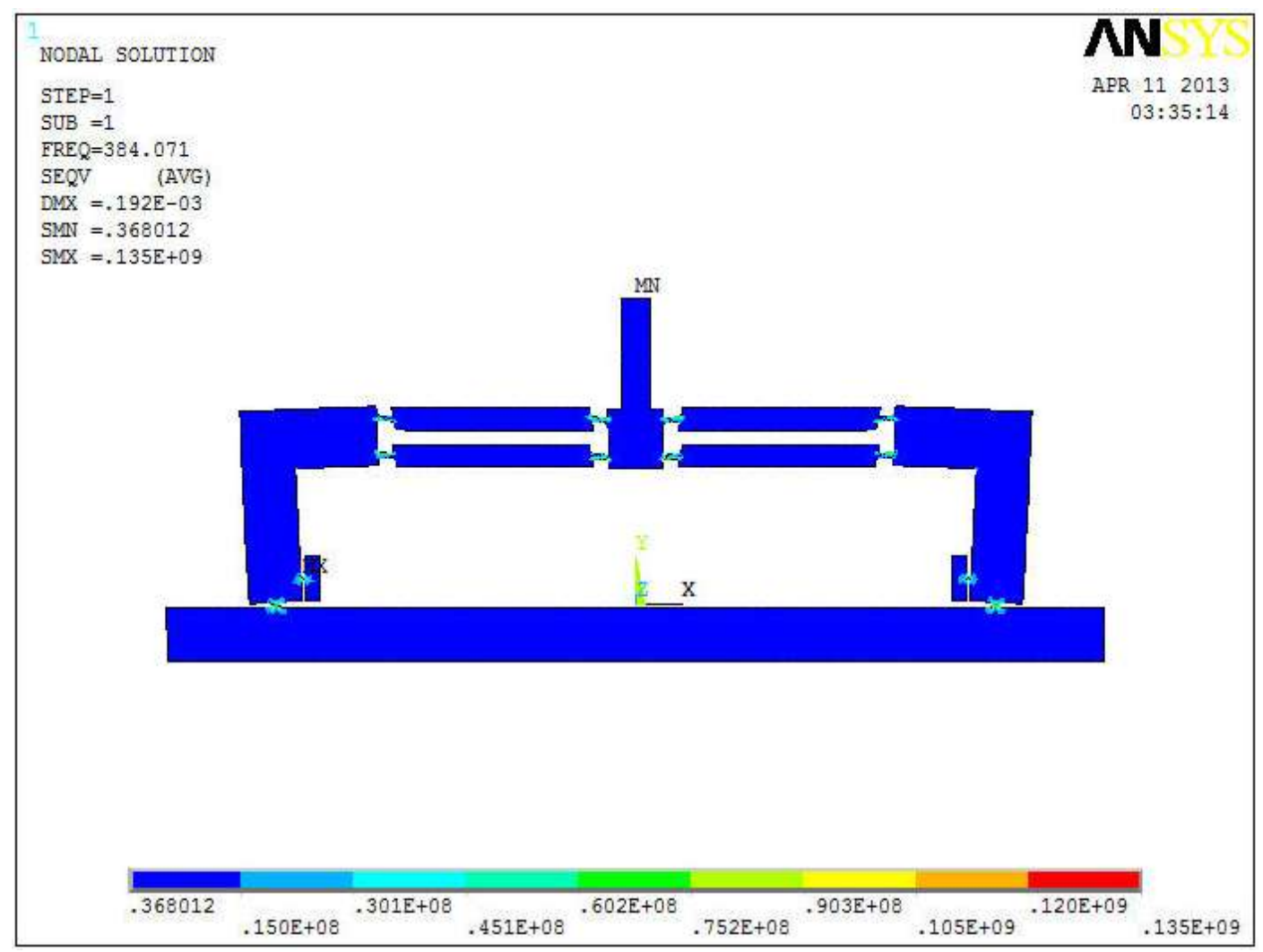

Figure 3.8. Contour plot of the Von-Mises stress of the optimized CMDA 


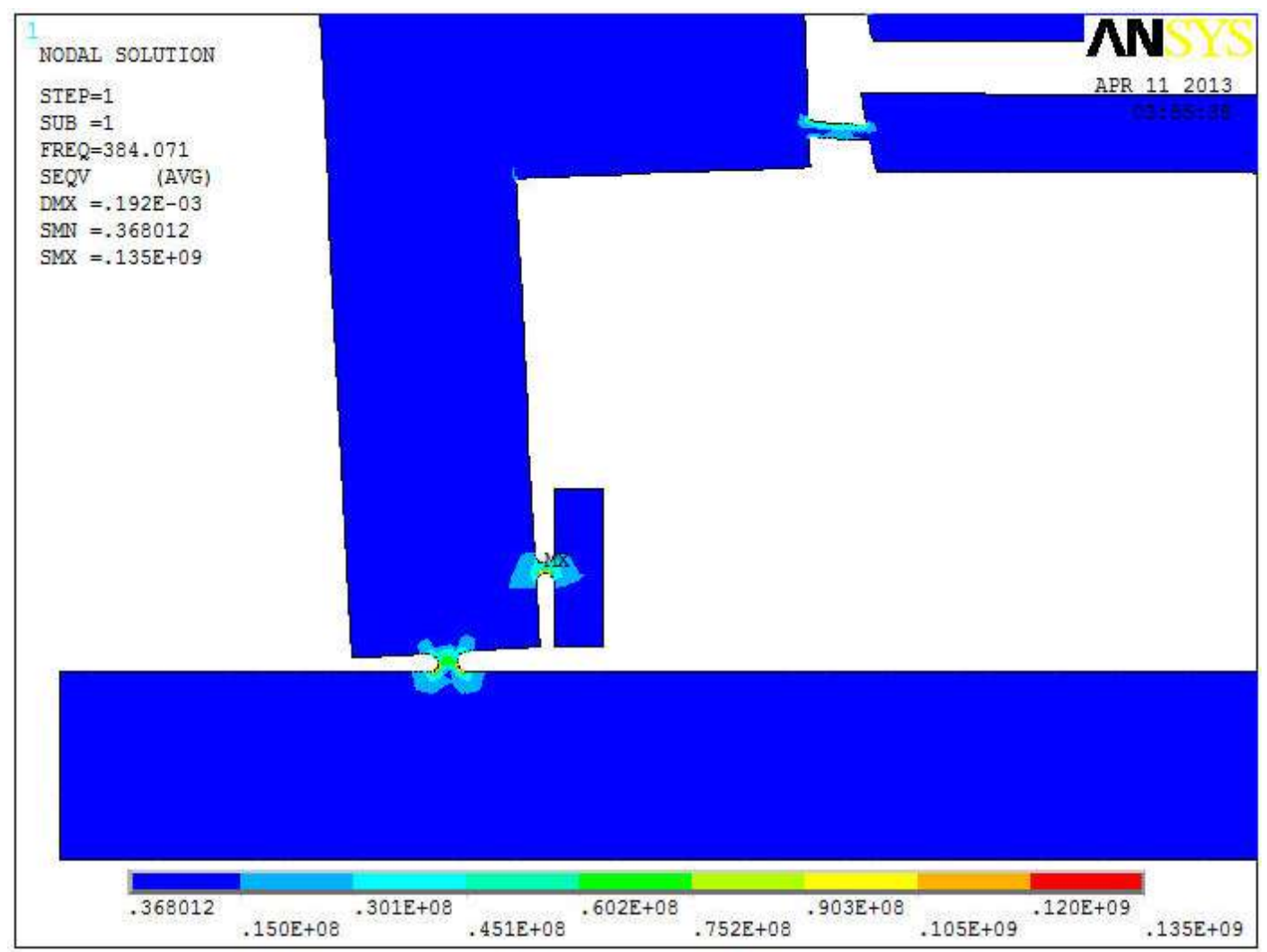

Figure 3.9 Contour plot of the Von-Mises stress of the left circular flexure hinge of the optimized CMDA.

From the optimized results, it can be observed that the AR of the device increased from 14.7 for the un-optimized CMDA to 19.2 for the optimized device. On the other hand, NF decreased from $475.54[\mathrm{~Hz}]$ for the initial design to $384.071[\mathrm{~Hz}]$ for the optimized design. Such a result is expected as the goal of the optimization is to achieve high AR value with a small sacrifice of NR. Figure 3.10 shows the corresponding mode shape to the found NF for the unloaded device. 

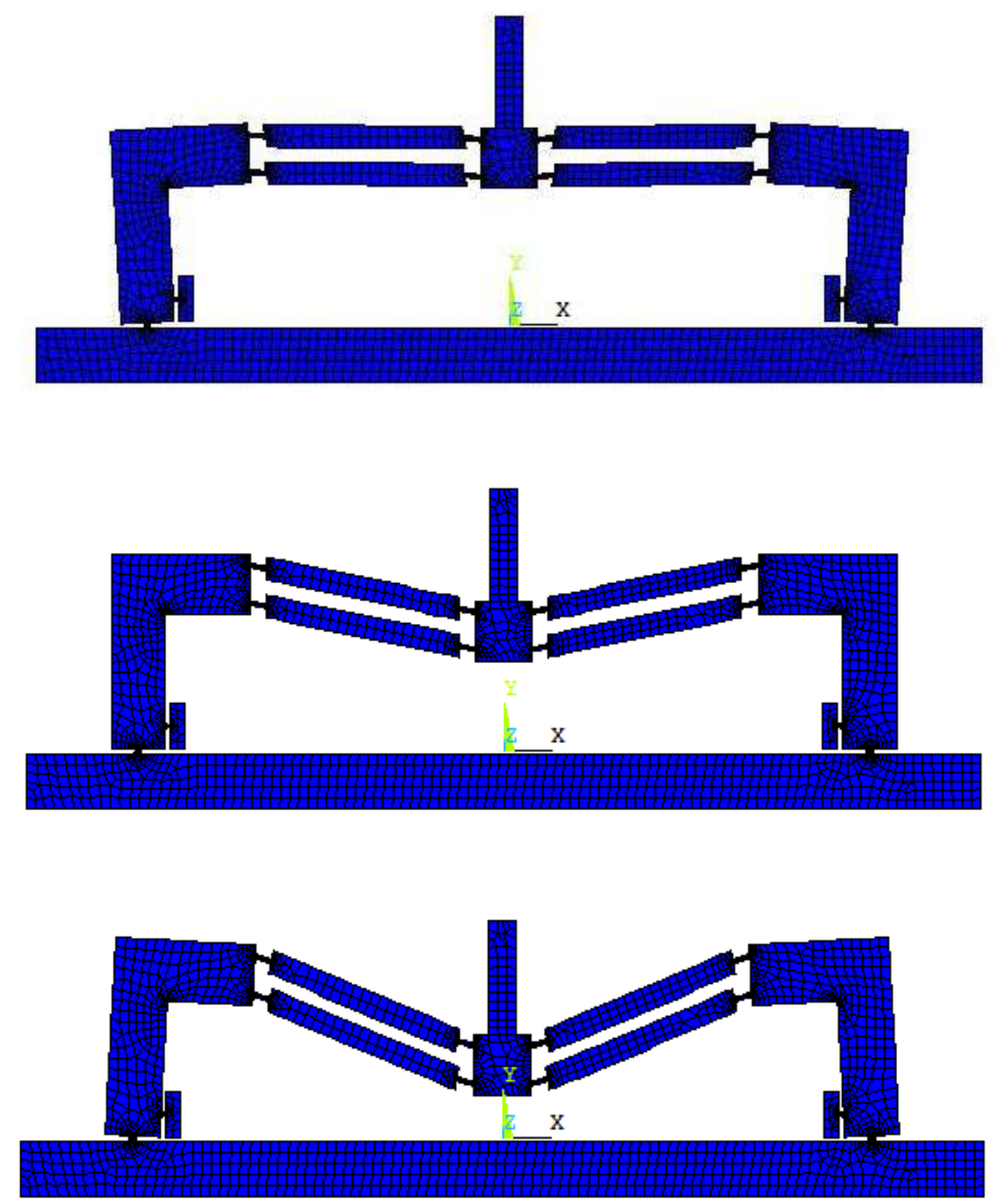

Figure 3.10 Mode shape corresponding to the optimized unloaded CMDA with 10 [ $\mu m]$ input displacement.

\subsubsection{Static and Dynamic FEM Analysis on the Optimized Design (Loaded CMDA for $274.61 g$ )}

In order to investigate and simulate the effects of the rigid endorectal coil assembled at the output of the proposed CMDA, the FEM static and dynamic analysis on the already 
optimized mechanism was performed again by adding a mass of $274.61[g]$ to the CMDA output. Figure 3.11 shows the FEM model of the optimized CMDA with an added mass on its output.

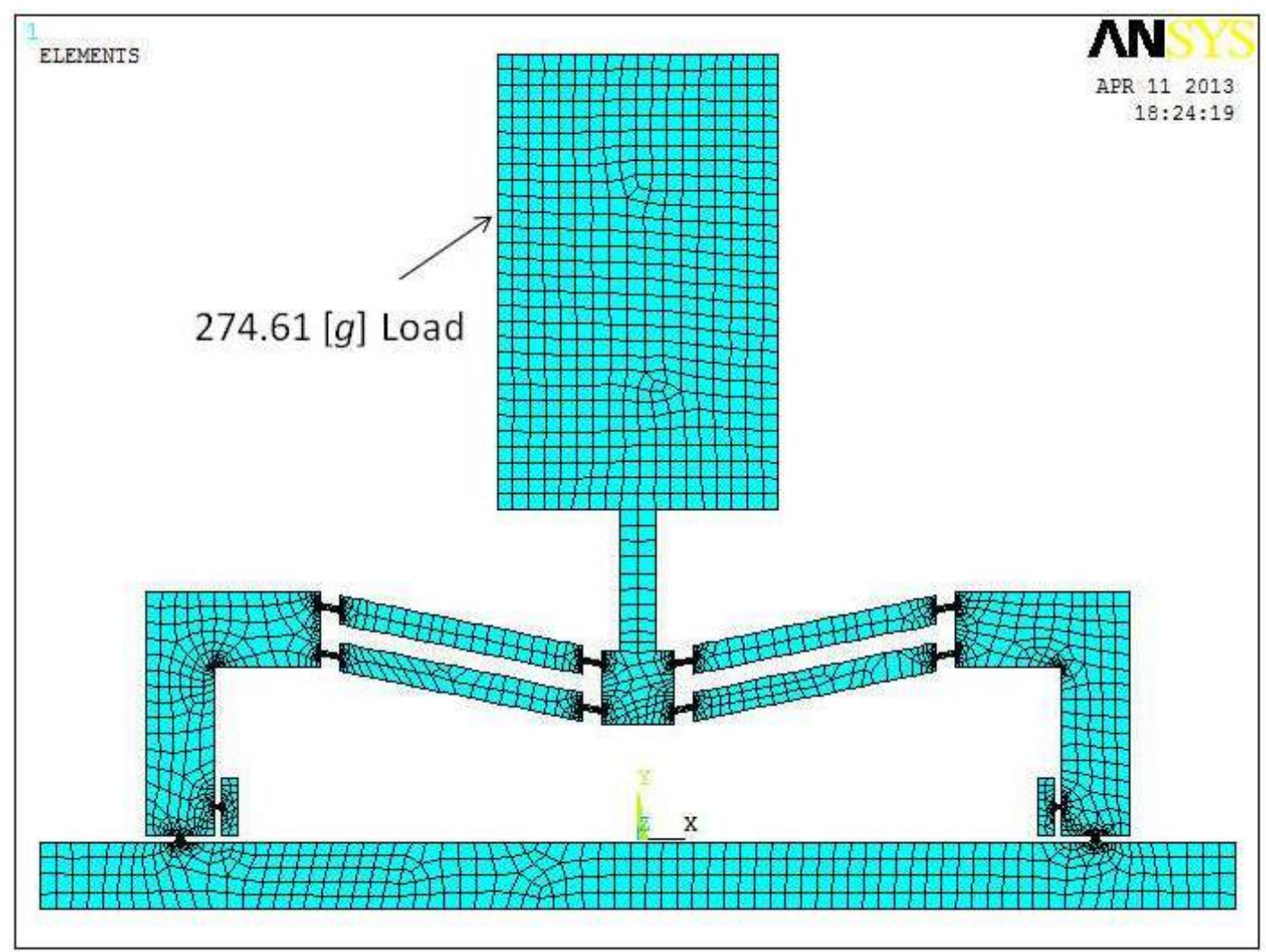

Figure 3.11 FEM model of optimized CMDA loaded for 274.61 [ $g$ ].

Static and dynamic FEM analyses to obtain the AR and NF of the loaded CMDA was then performed by applying input displacements of $10[\mu \mathrm{m}]$ to both of the device's two inputs. The results obtained from the above analysis are listed in Table 3.7.

Table 3.7: Initial Dynamic and Static Analysis Results

\begin{tabular}{ccc}
\hline NF $[\mathrm{Hz}]$ & AR & Max. Stress $[$ MPa $]$ \\
\hline 147.79 & 20.1 & 169 \\
\hline
\end{tabular}


As it can be seen from Table 3 and Figure 3.12, the loaded mechanism has an AR of 20.1. The increase in the AR of the loaded device in comparison to the unloaded one is due to the pre-loading effect that the added weight has on CMDA. In another word, the added weight pushes the mechanisms output further down and increases its displacement amplification potential. Figure 3.12 also illustrates that the maximum displacement of $201[\mu \mathrm{m}]$ occurs at the device's output when a displacement of $10[\mu \mathrm{m}]$ applied to CMDA inputs. The NF of the device however, was decreased from $475.54[\mathrm{~Hz}]$ for the unloaded case to $147.79[\mathrm{~Hz}]$ for the loaded one. This result was quite expectable since frequency is inversely proportional to mass. Figure 3.13 shows the corresponding mode shape to the found NF for the loaded device.

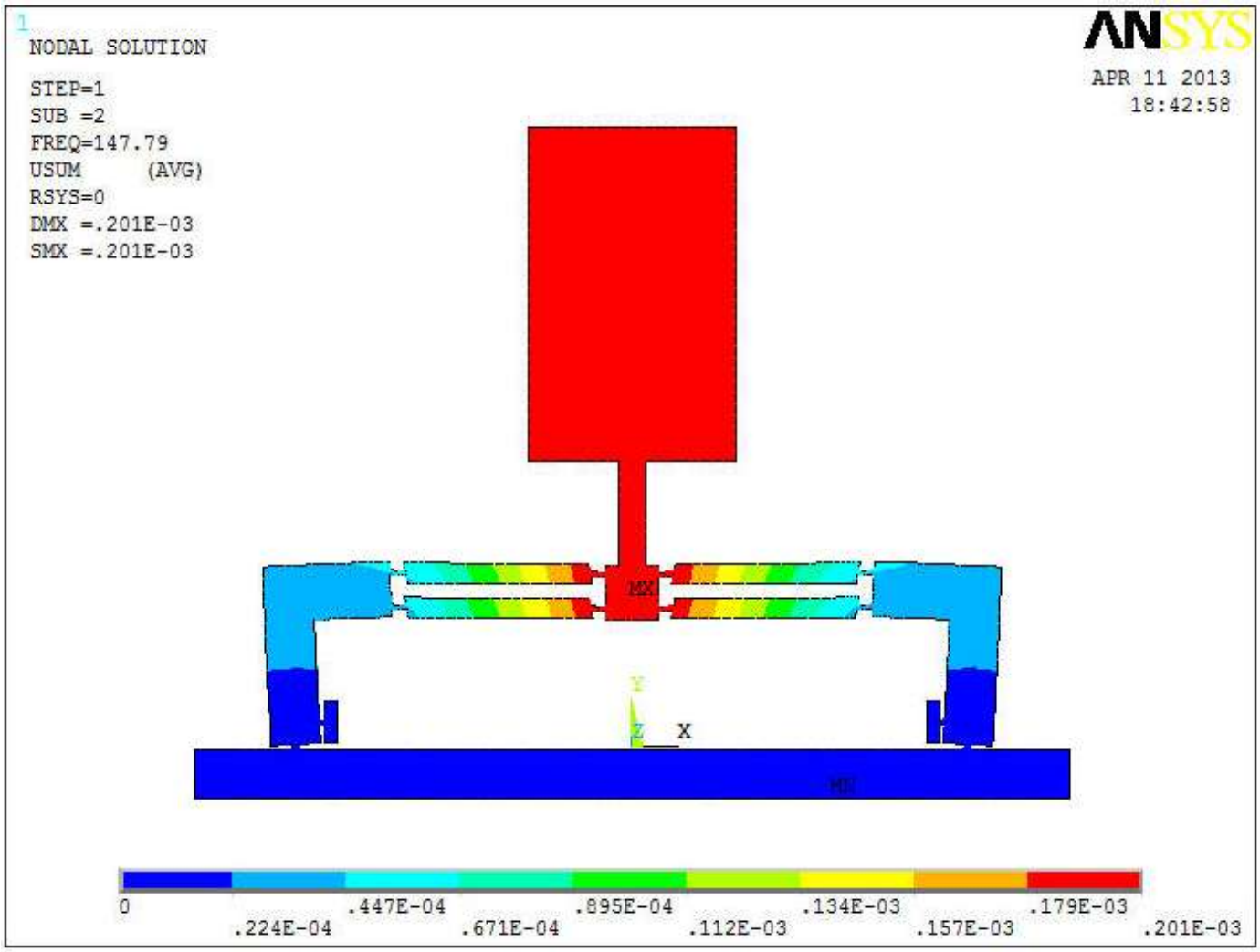

Figure 3.12 Contour plot of the displacement vector sum of the loaded CMDA design when 10 [ $\mu m$ ] input displacement is applied (unit: $\mathbf{m})$. 

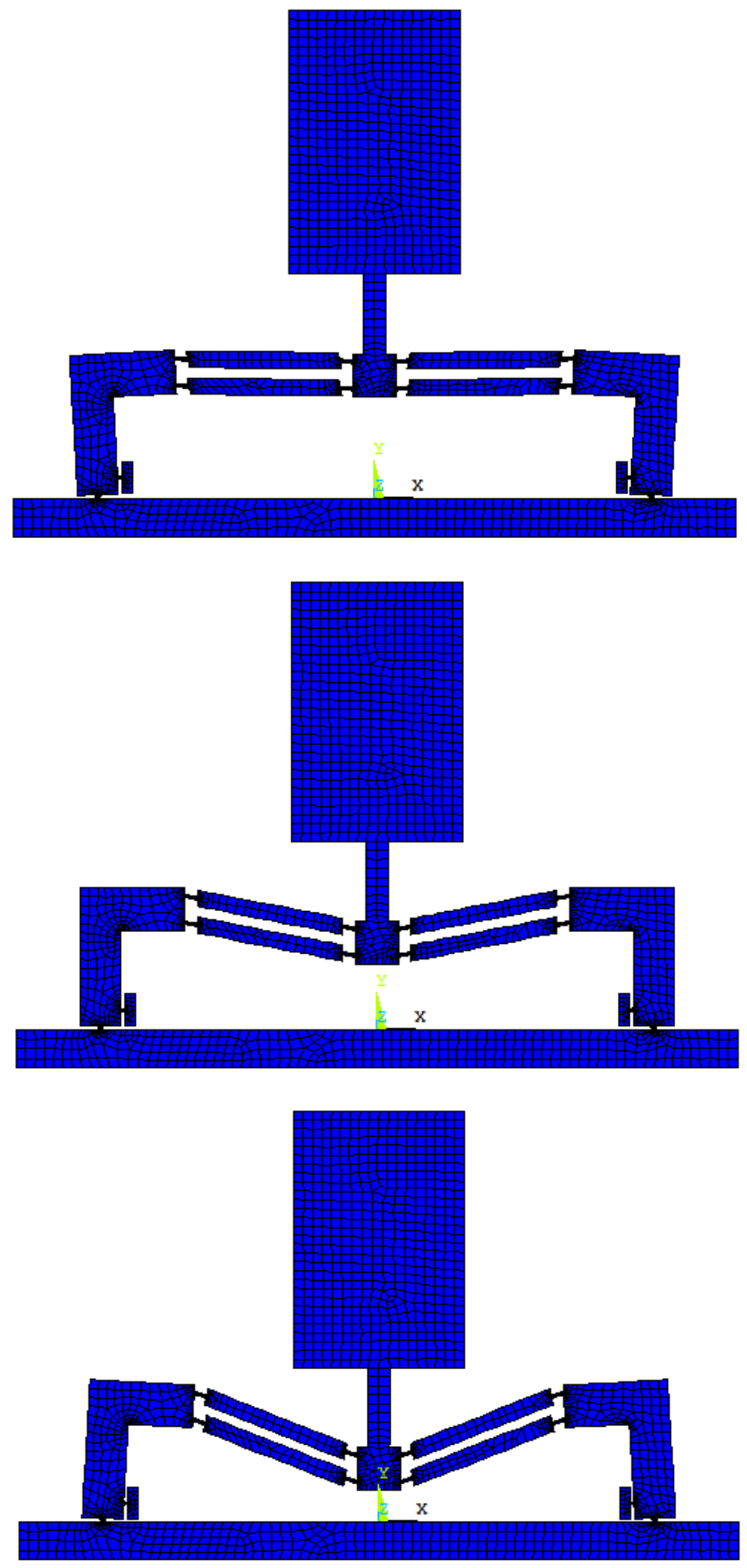

Figure 3.13 Mode shape corresponding to the optimized loaded CMDA with $10[\mu \mathrm{m}]$ input displacement. 
Figure 3.14 is the contour plot of the Von-Mises stress of the loaded CMDA when displacements of $10[\mu \mathrm{m}]$ were applied to its inputs. This figure illustrates that the loaded mechanism experiences a maximum stress of $169[\mathrm{MPa}]$ at the right filleted corner rectangular flexure hinge attaching the inclined top bar of the mechanism to its output. A close up image of the mentioned high stress are is shown if Figure 3.15. This high stress area is marked on the figure by $M X$. Certainly, the maximum stress is much less than the Yield Strength of the material

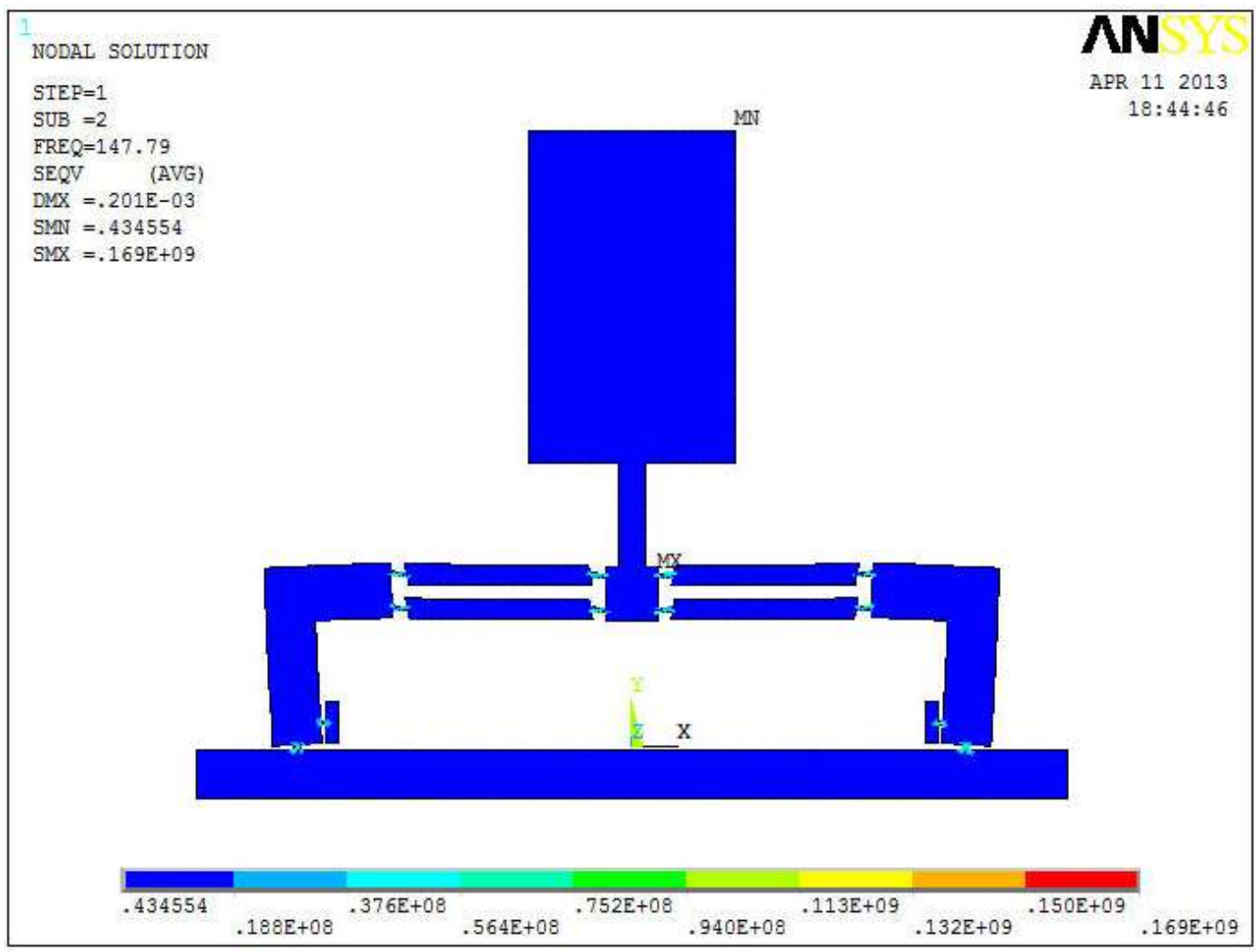

Figure 3.14 Contour plot of the Von-Mises stress of the loaded CMDA. 


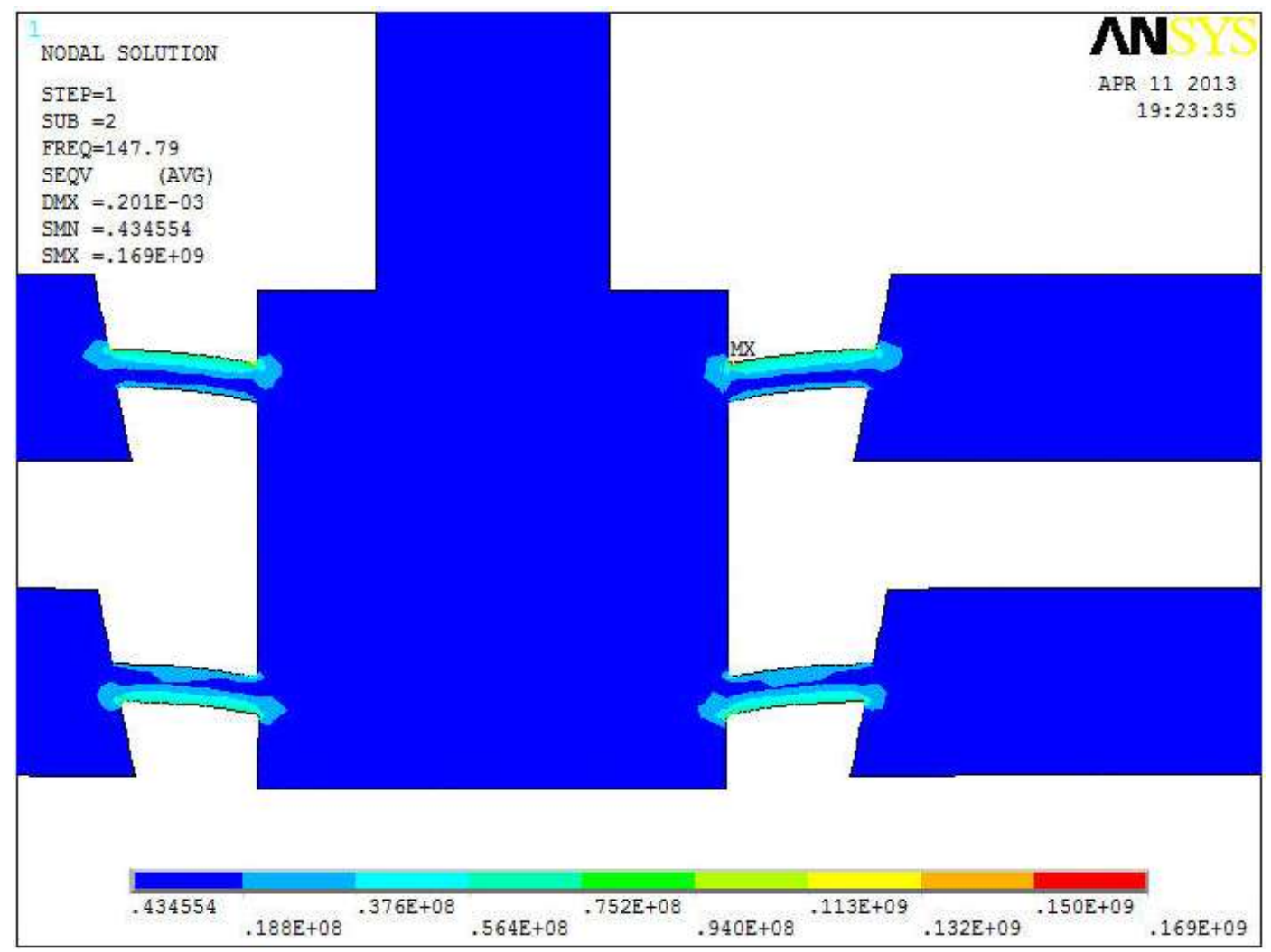

Figure 3.15 Contour plot of the Von-Mises stress of the right fillet corner rectangular flexure hinge of the loaded CMDA.

\subsubsection{Experiments for Unloaded and Loaded CMDA for Prostate MRE Application}

Based on the optimal parameters obtained from FEM analysis a prototype CMDA was built from brass using the wire EDM. Figure 3.16 shows the manufactured unloaded CMDA coupled with PICA ${ }^{\mathrm{TM}}$ P-010.80P PZT actuator. Figure 3.17 also shows the entire designed mechanism used for prostate MRE application. Please note that the CMDA mechanism along with the PZT actuator are placed in the white plastic casing while the output of the CMDA is mechanically coupled to the inflatable endorectal coil. 


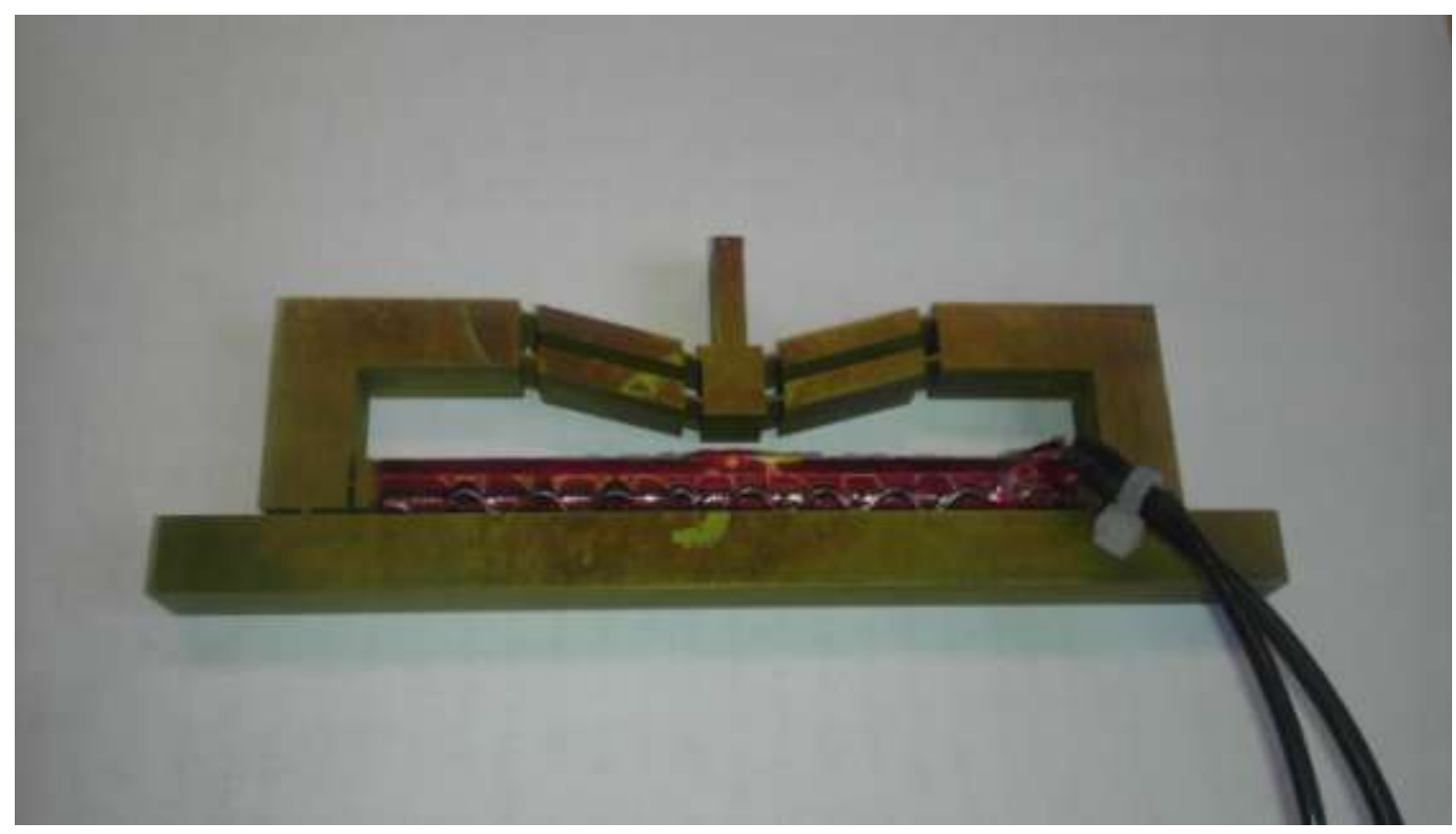

Figure 3.16 Manufactured CMDA integrated with $P I C A^{\mathrm{TM}} P-010.80 P$ PZT actuator, based on the optimized parameters obtained by FEM analysis (courtesy of Arvin Arani)..

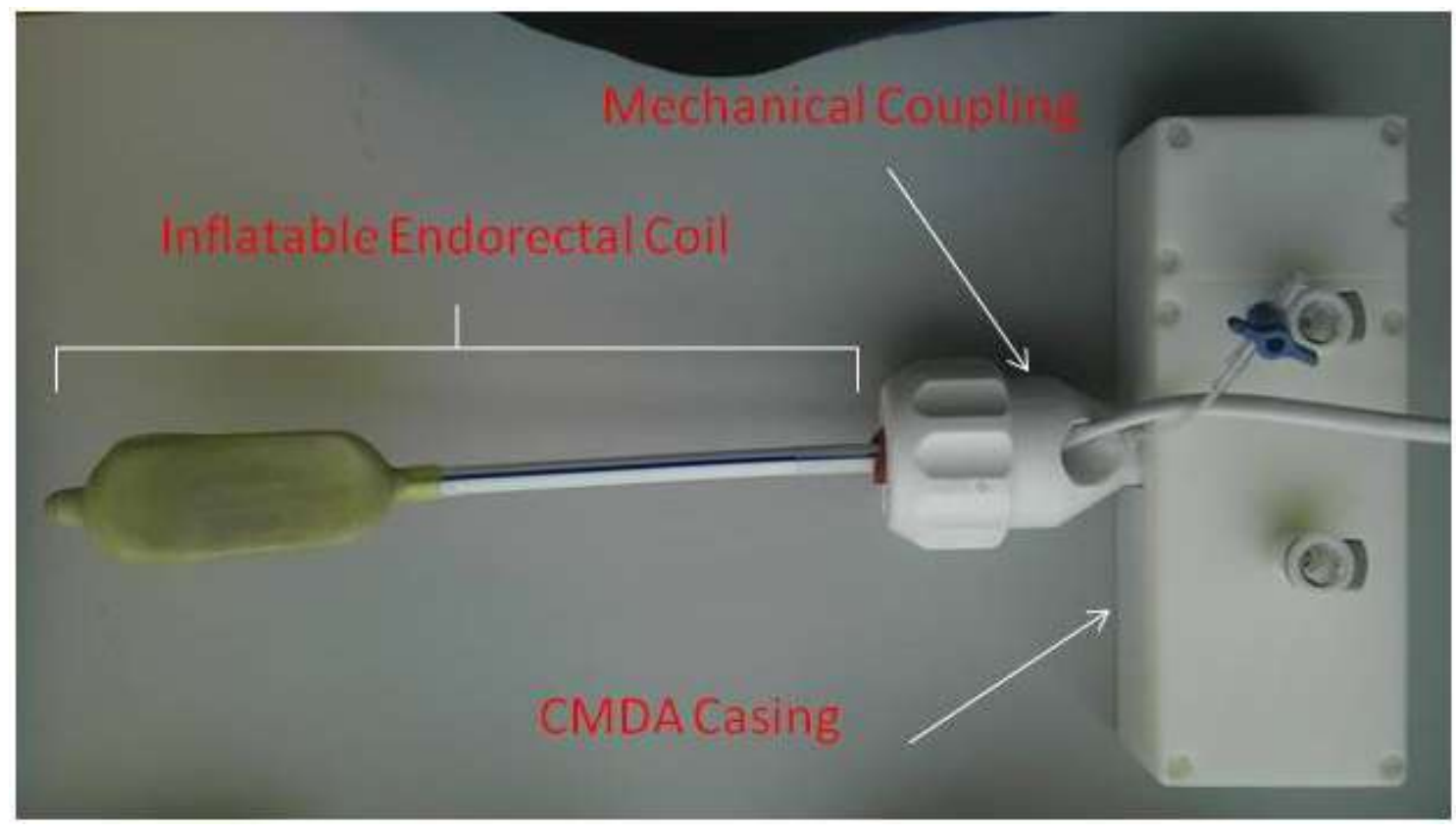

Figure 3.17 Entire designed mechanism used for prostate MRE application (courtesy of Arvin Arani).

The experiments setup and all the experimental data analysis were performed by University of Toronto Department of Bio-Medical Physics. Tests were done to find the dynamic and 
kinematic response on the designed optimal CMDA for both loaded and unloaded cases. Figure 3.18 is the plot of the Displacement Amplitude versus Frequency of both loaded and unloaded CMDA. As it can be observed from the figure the CMDA has a displacement amplitude of about $134[\mu \mathrm{m}]$ for an input displacement of $10[\mu \mathrm{m}]$ when it is unloaded. The obtained frequency for corresponding to the unloaded device is about $250[\mathrm{~Hz}]$. On the other hand, the loaded device possesses a displacement amplitude of $117.7[\mu m]$ with a frequency of $100[\mathrm{~Hz}]$. The obtained experimental data are not quite conforming to the ones obtained through FEM analysis. The occurred discrepancy could be due to fact of CMDA not having linear motions at their output. A 3-D FEM model of the designed CMDA can also yield better results than the 2-D one. Nevertheless, since the designed CMDA has no application in precision positioning theses discrepancies in FEM results and actual experimental results of no concern.

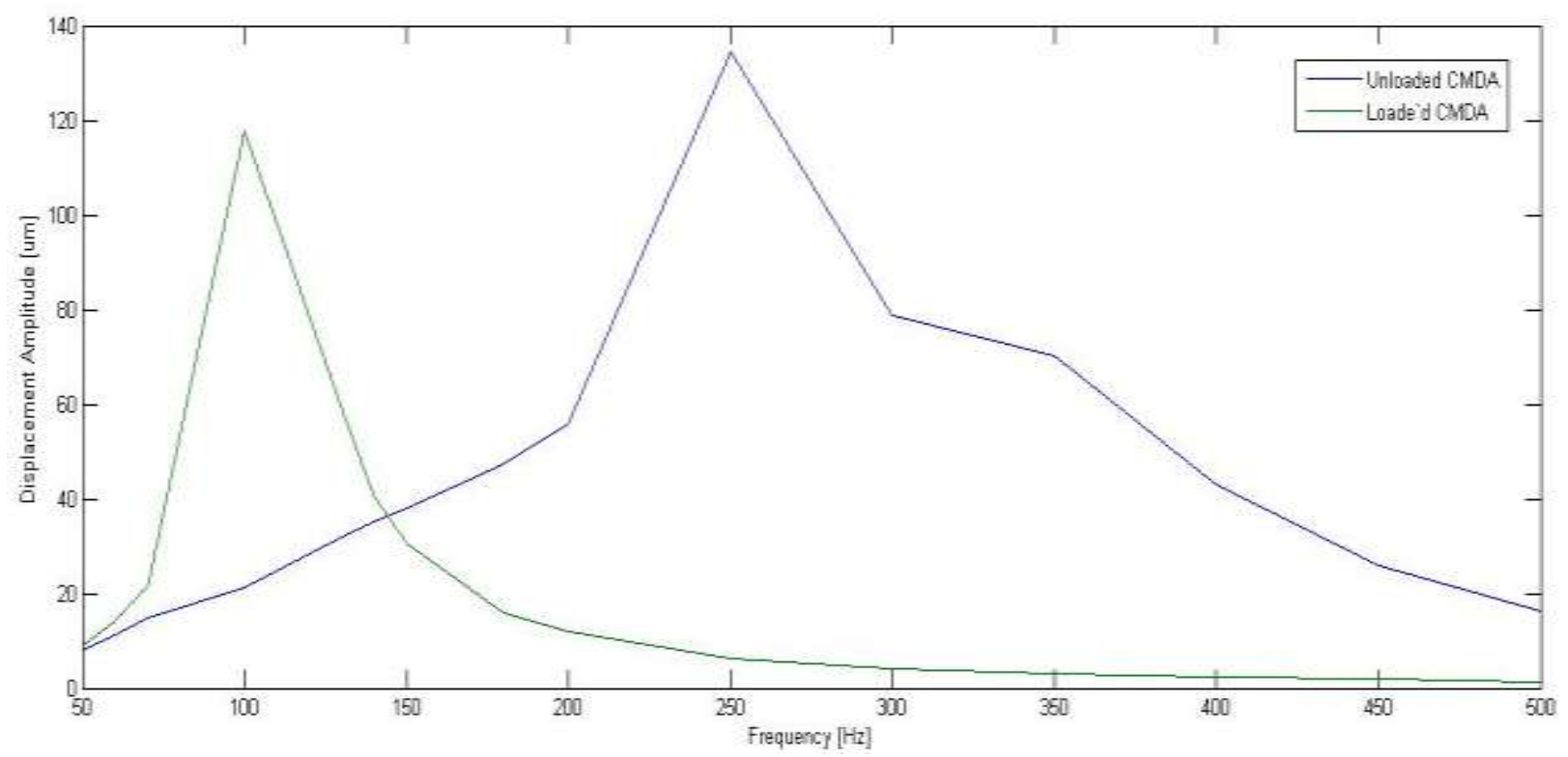

Figure 3.18 displacement amplification of loaded and unladed CMDA's vs. frequency (unpublished data, courtesy of Arvin Arani) 
Figure 3.19 also shows the displacement amplitude of the CMDA as a function of time. From the figure one can observe that as time passes the output displacement of the CMDA diminishes $180[s]$ and after that the output displacement keeps a constant value of about 150 $[\mu m]$. This is due to the transferred heat generated by the PZT actuator to the CMDA structure.

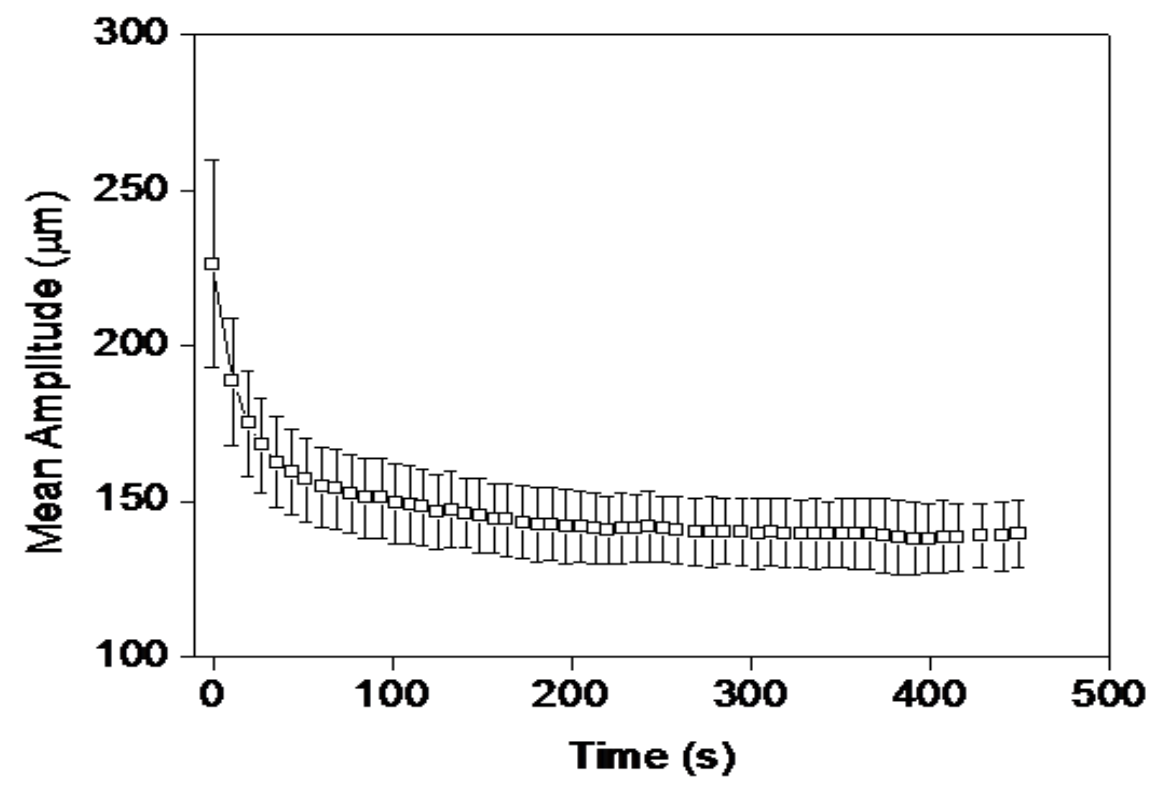

Figure 3.19 Displacement amplitude as a function of time.

The next experiment with the CMDA was conducted by coupling the device to an inflatable endorectal coil (Figure 3.17) and tests it on a mock up of a prostate in a gels environment, using a magnetic resonance imaging (MRI) unit. This test was proved to be successful since the displacement amplifications generated by the CMDA were adequate enough to send sufficient amount of vibrations into the prostate gland. Figure 3.20 illustrate the vibration generated using the designed CMDA integrated with the PZT actuator and the ones generated by the direct derive of the PZT with no amplification used. 


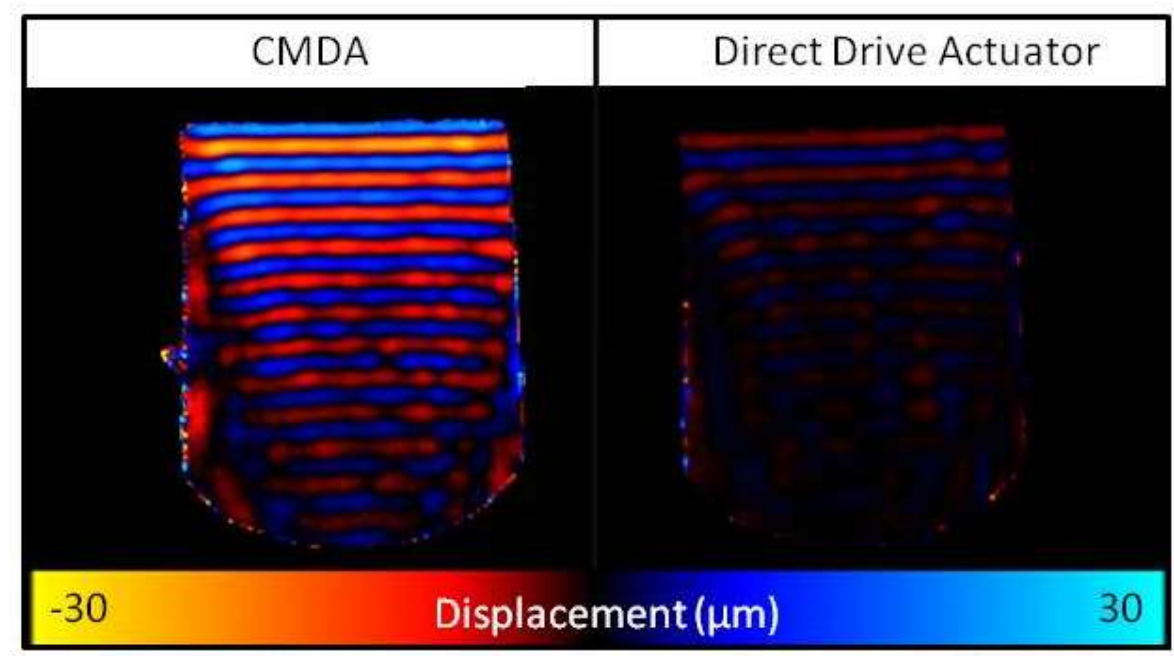

Figure 3.20 Fold amplification with CMDA (left) vs. using direct derive (right) (courtesy of Arvin Arani).

Finally Figure 2.21 shows the colored MRI image of the mock up prostate gland in the gel environment. One can release from this image that the location of the inclusions (i.e. suspicious cancerous tissues) are well illustrated in the MRI picture. This image is possible since the vibration generated by the PZT actuator is amplified enough, so the waves can travel deep into the prostate gland and reveal the location of the stiffer (i.e. suspicious cancerous tissues) tissues.

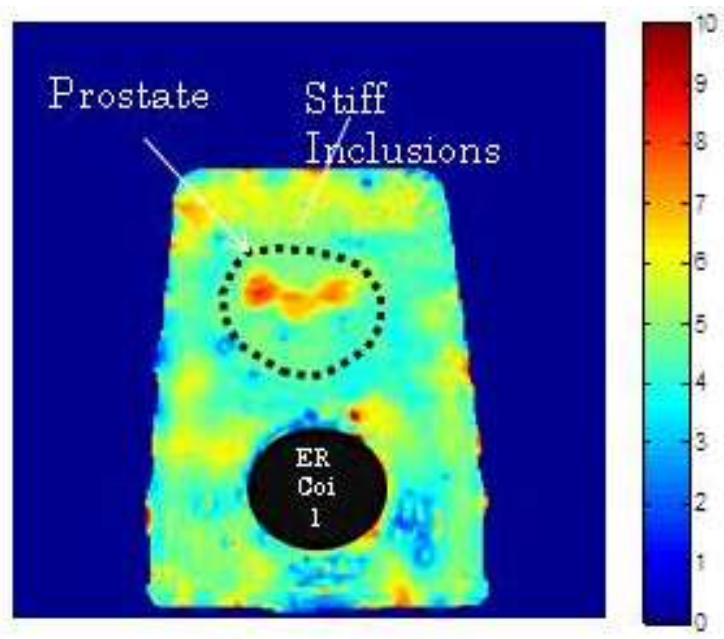

Figure 3.21 MRI image of the prostate gland with the location of stiff inclusions shown (courtesy of Arvin Arani). 


\subsubsection{Conclusion}

In this chapter design, development, analysis and optimization of a CMDA for the use in prostate MRE application were discussed. Detailed FEM static and dynamic analysis for the initial design of the CMDA was provided. It was then shown that by a proper approach to optimization, the dynamic and static characteristics of the CMDA can be tailored towards a better performance depending on the application. The MRI test of the CMDA proved the entire requirements were met and the proposed design was a success.

Note: All the ANSYS® codes used for designing, analysing, and optimizing of the unloaded and loaded CMDA provided in Appendix B. 


\section{DESIGN AND OPTIMIZATION OF A XY COMPLIANT MECHANICAL DISPLACEMENT AMPLIFIER}

\subsection{Introduction}

In this chapter, a new design for a XY planar motion compliant mechanical displacement amplifier (CMDA) based on the design of a symmetric five-bar compliant mechanical amplifier is introduced. As it is mentioned earlier, the symmetric five-bar structure CMDA is only capable of generating an output displacement constrained in only one direction. However, many industrial applications of micro motion devices require manipulators be capable of generating an XY planar motion with high NF. The goal of this research is to exploit the advantages of the symmetric five-bar structure CMDA and propose a novel XY CMDA capable of generating large displacements in both $\mathrm{X}$ and $\mathrm{Y}$ directions. Detailed analysis with Finite Element Method (FEM) of static and dynamic characteristics of the proposed XY CMDA design is also provided. Finally, the optimization process and results to increase the Amplification Ratio (AR) of the proposed XY compliant mechanism with minimal compromise in Natural Frequency (NF) is discussed.

\subsection{Design Requirements and Constraints}

The proposed planar motion CMDA comprises of two identical legs located in $\mathrm{X}$ and $\mathrm{Y}$ directions as shown in Figure 4..1 Each leg then is connected with a pair of arms to the output of the device. Leg A of the device translates the horizontal driving force and motion of the PZT to the Y direction motion, while the Leg B does that in the $\mathrm{X}$ direction motion. 
Please note that both legs A and B are each constrained in all degree of freedoms from the base.

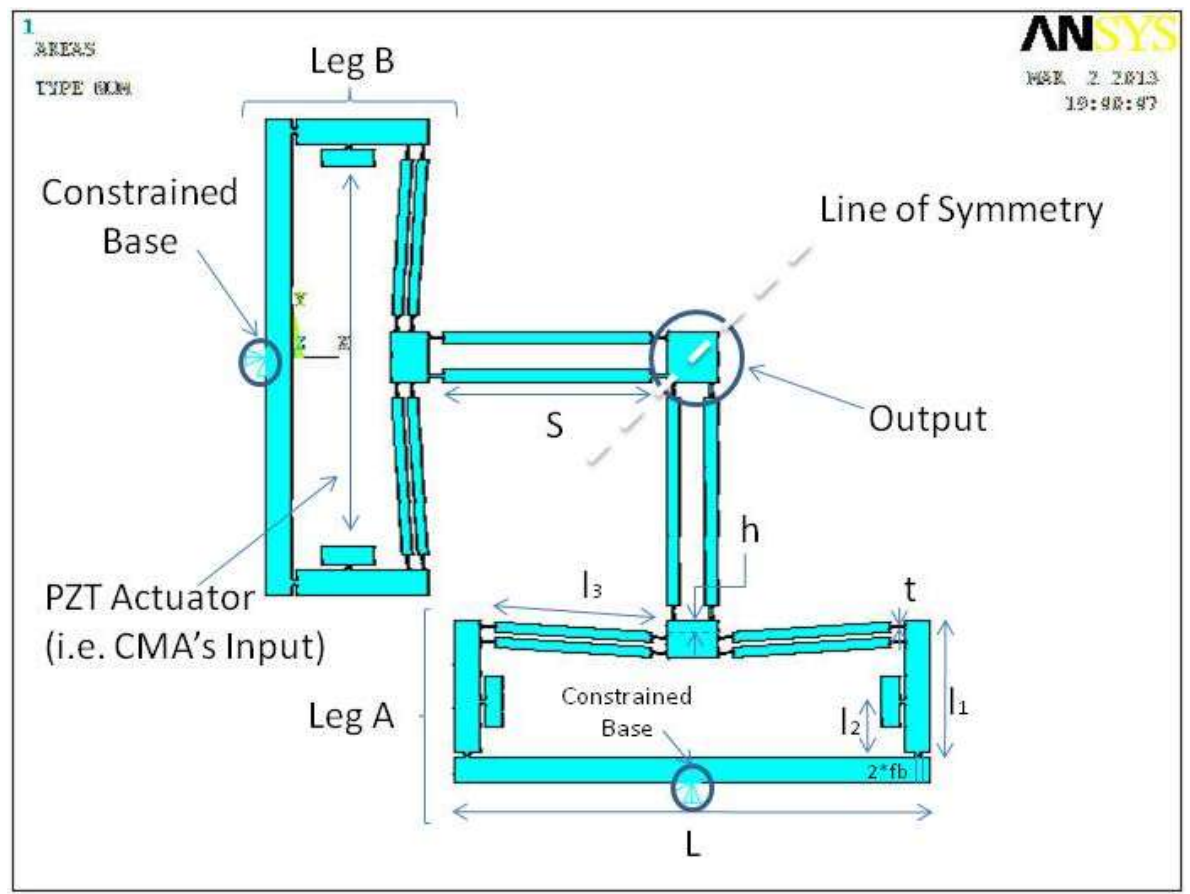

Figure 4.1 Planar motion generator CMDA based on symmetric five-bar topology

According to Figure 4.1, the proposed planar motion generator CMDA has the following characteristics:

(1) The mechanism is a symmetric compliant mechanism with two degree of freedoms (DOF) due to two identical legs in $\mathrm{X}$ and $\mathrm{Y}$ directions.

(2) A CMDA is applied to amplify the stroke of a PZT actuator for each leg.

(3) The designed mechanism can be used to generate both planar and linear micro motions.

(4) The moving output or end-effector of the device is design to be a cube.

(5) Each leg of the planar motion generator CMDA consists of two pairs of the inclined bars of the length $l_{3}$ to increase the NF of the designed mechanism. 
Some of the key parameters that have significant effect on the performance of the CMDA are also shown in Figure 4.2. Please note that the parameters shown on the Leg A can be used to construct the rest of the device since the mechanism is symmetric about the illustrated line of symmetry.

Table 4.1 below also lists the initial values of the most important parameters used to design the planar motion generator CMDA. Please note that the minimum dimensions that parameters $l_{2}$ and $L$ can adopt are related to the dimensions of NPA50SG actuators that will be integrated within each leg. $\mathrm{S}$ is determined to make two directional motions possible in a compact size. Parameter $h$ also stipulates the inclined angle of the double parallel bars, referring to Figure 2.9. Finally parameters $t$ and $f b$ represent the thickness of the rectangular and circular flexure hinges, respectively. The thickness of the CMDA is $10[\mathrm{~mm}]$.

Table 4.1: Design parameters of the planar motion generator CMDA (unit: $\mathbf{m m}$ )

\begin{tabular}{lcccccccc}
\hline Parameters & $L$ & $l_{l}$ & $S$ & $t$ & $l_{2}$ & $h$ & $f b$ & $l_{3}$ \\
\hline \multirow{2}{*}{ Dimension } & 93 & 26.696 & 40.5 & 0.35 & 11 & 2.5 & 0.35 & 30.57 \\
\hline
\end{tabular}

\subsection{Initial FEM Static and Dynamic Analysis}

A 3-D model of the planar motion generator CMDA was constructed using ANSYS ${ }^{\circledR}$ Mechanical APDL with the primary parameters listed in Table 4.1. Then by assigning the stainless steel material properties (Table 4.2) to the model, the CMDA was meshed using SOLID 186 element type. SOLID186 is a higher order 3-D 20-node solid element that exhibits quadratic displacement behavior. The element is characterized by 20 nodes having three translational degrees of freedom per node in the nodal $X, Y$, and $Z$ directions. This 
element supports plasticity, hyperelasticity, creep, stress stiffening, large deflection, and large strain capabilities (ANSYS, 2009).

Table 4.2: Stainless Steel Material Properties

\begin{tabular}{cc}
\hline Modulus of Elasticity $[\mathrm{GPa}]$ & 200 \\
Density $\left[\mathrm{kg} / \mathrm{m}^{3}\right]$ & 8000 \\
Poisson's Ratio & 0.285 \\
Yield Strength $[\mathrm{MPa}]$ & 703 \\
\hline
\end{tabular}

The initial static and dynamic FEM analyses for the following four cases are performed:

Case 1: Input displacements of $10[\mu \mathrm{m}]$ were applied to each of the device's four inputs to obtain the mechanism's overall AR and NF.

Case 2: Input displacements of $10[\mu \mathrm{m}]$ were applied to each of the two inputs of the Leg A of the device only to get the AR of the mechanism in vertical direction.

Case 3: Input displacements of $10[\mu \mathrm{m}]$ were applied to each of the two inputs of the Leg B of the device only to get the AR of the mechanism in horizontal direction.

Case 4: Two forces of $5[N]$ each were applied to the devices output (as shown in Figure 4.2) while an input displacement of $10[\mu \mathrm{m}]$ were applied to each of the device's four inputs. Then the mechanism's overall AR and NF were obtained by performing FEM analysis.

The cross-talk of the CDMA was calculated using the following two formulas (Sun, Zhang, Cheng, \& Zhang, 2011):

$$
\begin{gathered}
A_{y x}=\frac{\Delta y}{\Delta x_{s}} \\
A_{x y}=\frac{\Delta x}{\Delta y_{s}}
\end{gathered}
$$


where $\Delta y$ is the desired displacement in the $Y$ direction and $\Delta x_{s}$ is the spurious displacement found in $X$ direction when attempt to displace in $Y$, while in the case of $A_{x y}, \Delta x$ is the desired displacement in the $X$ direction and $\Delta y_{s}$ is the spurious displacement found in $Y$ direction when attempt to displace in $X$.

The results obtained from the above analysis are listed in Table 4.3.

Table 4.3: Initial Dynamic and Static Analysis Results

\begin{tabular}{cccc}
\hline Case Number & NF $[\mathrm{Hz}]$ & AR & Cross-Talk \\
\hline 1 & 293.09 & 33.3 & --- \\
2 & 289.27 & 24.3 & 19.5 \\
3 & 289.27 & 24.3 & 19.5 \\
4 & 293.09 & 27.6 & --- \\
\hline
\end{tabular}

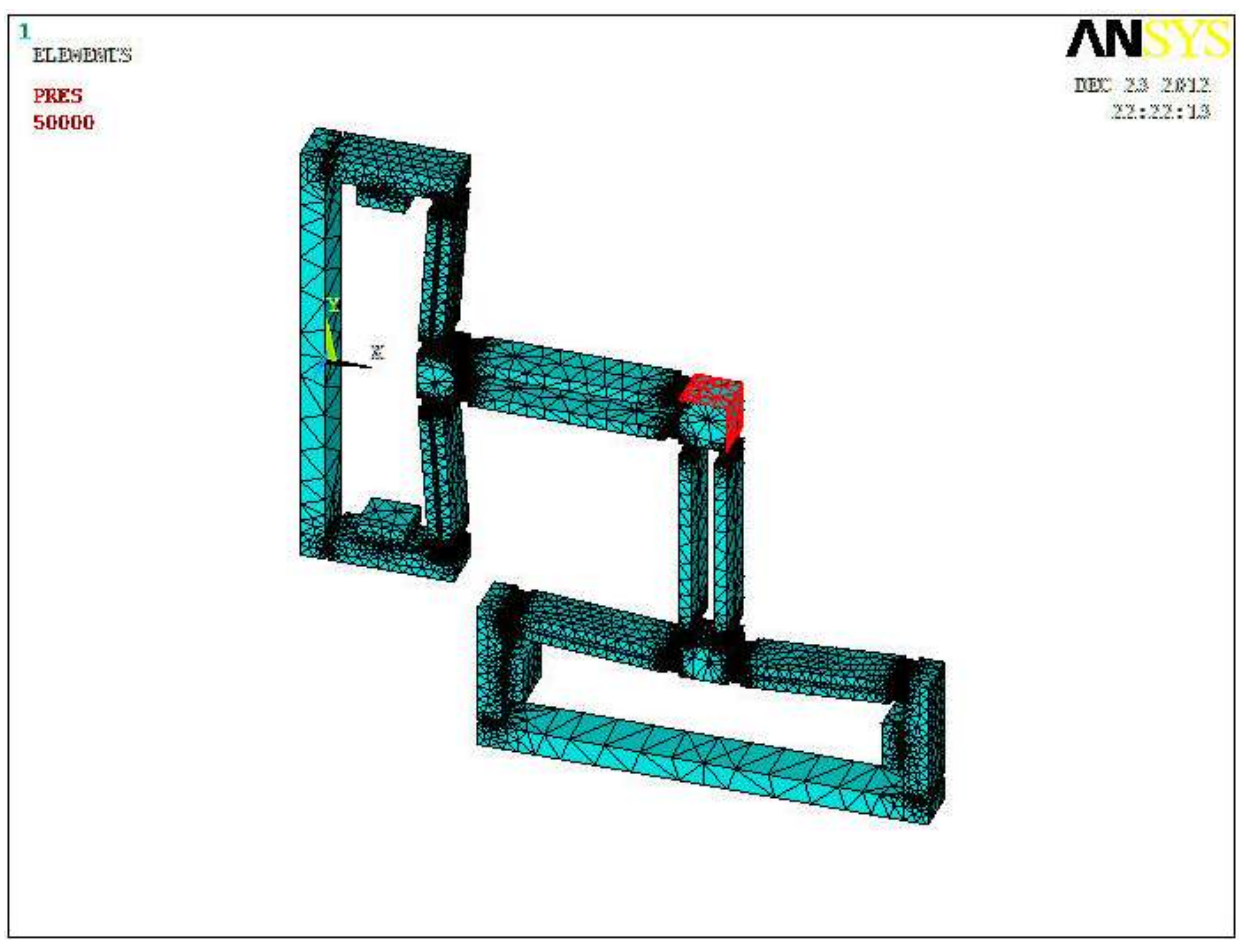

Figure 4.2 FEM model of the planar motion generator CMDA with the locations of the applied forces shown in red. 
As it can be seen from Table 4.3 the mechanism has an overall AR of 33.3 and a NF of 293.09 $[\mathrm{Hz}]$. The NF of the system remains the same for Case 4. However; the AR reduces from 33.3 for Case 1 to 27.6 for Case 4 due to device being under loading for this scenario, as the external force will act to resist the output motion of the CMDA. Table 4.3 also shows that the mechanism possess a NF of $289.286[\mathrm{~Hz}]$ and an AR of 24.3 in each vertical and horizontal directions when only one direction motion is produced using only one PZT actuator. The cross-talk level between $Y$ and $X$ displacement as well as the $X$ and $Y$ displacement for both cases 2 and 3 were also found to be 19.51 .

Figure 4.3 illustrates the contour plot of the Von-Mises stress of the CMDA when displacements of $10[\mu \mathrm{m}]$ were applied to all of its inputs. From the figure, it can be observed that the CMDA experiences a maximum stress of $124[\mathrm{MPa}]$ at the rectangular flexure hinge attached to the left vertical arm that connects the Leg A of the device to cubic output. This high stress area is marked on the figure by $M X$. Certainly, the maximum stress is much less than the Yield Strength of the material.

It should be mentioned that this initial design is a sub-optimal design based on previous one dimensional CMDA presented by Ouyang et al. (2008), and it will be used as the first iteration in the following optimal design process. 


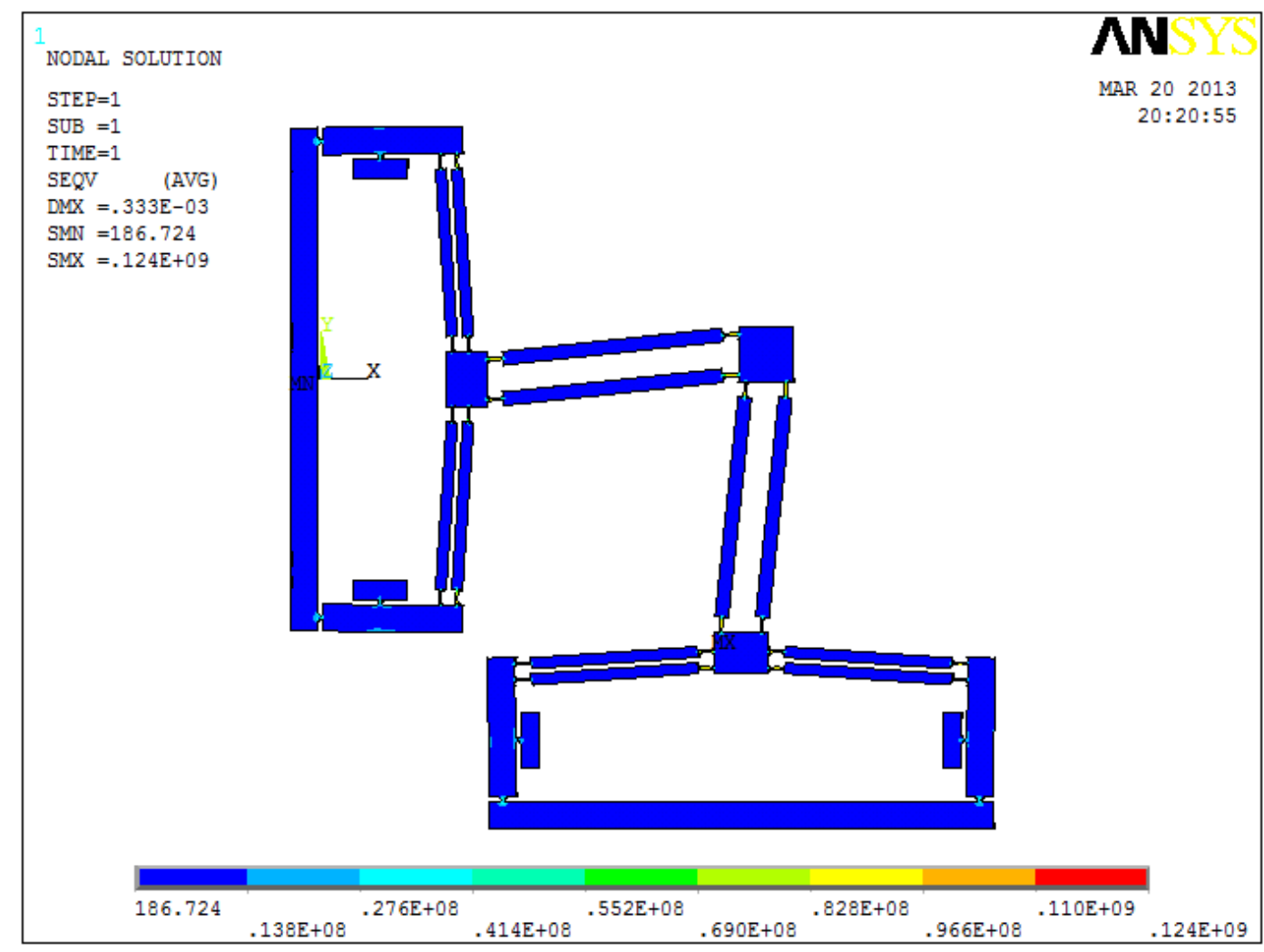

Figure 4.3 Contour plot of the Von-Mises stress of the initial CMDA.

\subsection{Optimal Design}

\subsubsection{Design Parameters}

The goal of an optimization process for the planar motion generator CMDA is to obtain the values of some significant design parameters that maximize the AR of the device with taking into account the maximum stress occurred in the flexural hinges and the maximum force available by PZT actuators to create a desired input displacement. These significant parameters were discovered by Ouyang et al. (2008) and were regarded as having the most effects on the AR of the symmetric five-bar CMDA. Table 4.4 lists the design parameters used for the optimization of the planar motion generator CMDA with their corresponding 
allowable ranges. It should be noted that selected ranges of the design parameters are based on some sub-optimal design results.

Table 4.4: Design Parameters and their range used for optimization (unit: $\mathbf{m m}$ )

\begin{tabular}{cc}
\hline Design Parameter & Range \\
\hline$l_{1}$ & $26.696-28.700$ \\
$\mathrm{t}$ & $0.300-0.500$ \\
$\mathrm{l}_{2}$ & $10.500-12.500$ \\
$\mathrm{~h}$ & $2.000-3.000$ \\
$\mathrm{fb}$ & $0.250-0.400$ \\
\hline
\end{tabular}

\subsubsection{Optimization Process}

As mentioned earlier, one of the optimization constraints is the maximum stress that flexural hinges can undergo. This was defined by stainless steel yield strength listed in Table 4.2. In another word during optimization process, the maximum stress $(\sigma)$ experienced within flexural hinges was not allowed to exceed stainless steel yield strength $\left(\sigma_{y}\right)$ with a safety factor (SF) which was chosen to be one. Another constraint considered for the optimization was the force required to create a given amount of input displacement. This force also was set to not exceed NPA50SG actuators push load capacity of $1000[N]$ during the optimization process.

Therefore, the optimization problem of the XY motion CMDA can be described as:

$$
\text { Maximum: } \quad A R=y / \delta
$$

\section{Subject to:}

$$
\sigma<\sigma_{y} / S F
$$




$$
F+(20 E 6) *(\delta)<1000
$$

where $F$ is the force required to create a displacement at each of the device's input, and the constant 20E6 in Equation 4.4 is of the actuator inherent stiffness.

As there is no explicit formula to build the connections between the design parameters and the objective function and constraints, a numerical optimization analysis using ANSYS is approached. ANSYS first order optimization method in conjunction with random design method were used to find the design parameters (within the given range) that yields the best AR for the proposed design for a constant $10[\mu \mathrm{m}]$ input displacement, while two constraints are satisfied at the same time. The optimized parameters under this specific condition are obtained through ANSYS and listed in Table 4.5.

Table 4.5: Design Parameters obtained after optimization (unit: $\mathrm{mm}$ )

\begin{tabular}{cc}
\hline Design Parameter & Value \\
\hline $\mathrm{l}_{1}$ & 26.696 \\
$\mathrm{t}$ & 0.303 \\
$\mathrm{l}_{2}$ & 10.526 \\
$\mathrm{~h}$ & 2.090 \\
$\mathrm{fb}$ & 0.347 \\
\hline
\end{tabular}

Figure 4.4 is the plot of the design parameters versus the design objective (DMAX). As it can be seen from the graph and Table 5 design parameters $l_{1}, l_{2}$, and $h$ have the most effect on the AR of the device. On the other hand, parameters $f b$ and $t$ maintained relatively constant values throughout the optimization process. The thickness $t$ of the rectangular flexure hinge is very close to the minimum value of the allowable range, while the thickness $f b$ of the circular flexure hinge is determined mainly by the allowable Please note that smaller values 
of these two parameters yield a higher AR. However, thinner flexure hinges can sustain less stress for a given input displacement and will result in the CMDA failure.

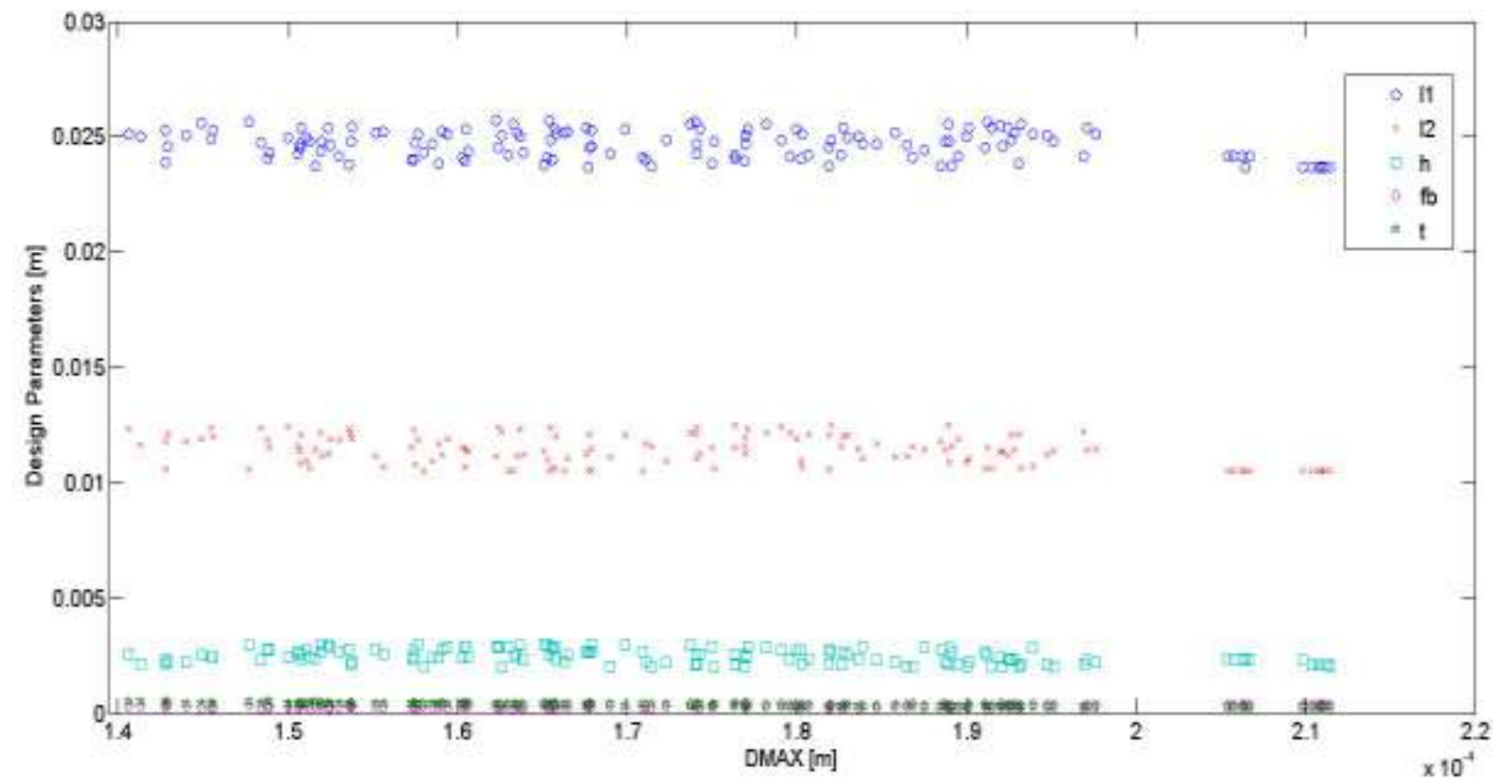

Figure 4.4 Plot of design parameters versus design objective (unit: $\mathrm{m}$ )

\subsubsection{Static and Dynamic FEM Analysis on the Optimized Design}

After obtaining the optimized design parameters, static and dynamic FEM analysis were performed on the proposed optimized CMDA design for four different cases. These analyses were done for the same four cases as of the initial design. Table 4.6 lists the dynamic and static results of the proposed optimized design for each case.

Table 4.6: Optimized Dynamic and Static Analysis Results

\begin{tabular}{cccc}
\hline Case Number & NF $[\mathrm{Hz}]$ & $\boldsymbol{A R}$ & Cross-Talk \\
\hline 1 & 243.91 & 39.2 & --- \\
2 & 239.86 & 28.5 & 15.52 \\
3 & 239.86 & 28.5 & 15.52 \\
4 & 243.91 & 32.8 & --- \\
\hline
\end{tabular}


Figure 4.5 illustrates the contour plot of the Von-Mises stress of the optimized CMDA when displacements of $10[\mu \mathrm{m}]$ were applied to all of its inputs. From this figure, it can be observed that the location of the maximum stress experienced by the CMDA has changed to the location of rectangular flexure hinge attached to the top horizontal arm $S$ that connects the Leg B of the device to cubic output. This high stress area is shown on the figure by MX and the maximum stress experienced by the hinge is $132[\mathrm{MPa}]$. Such an increase in the amount of the stress experienced by the rectangular flexure hinge is due to the fact that the thickness of the hinge has reduced from $0.35[\mathrm{~mm}]$ for the un-optimized CMDA to 0.303 $[\mathrm{mm}]$ for the optimized one. The same reason has also led to the decrease of the cross-talk level between $Y$ and $X$ displacement as well as the $X$ and $Y$ displacement from 19.51 for the un-optimized case to 15.52 for the optimized one.

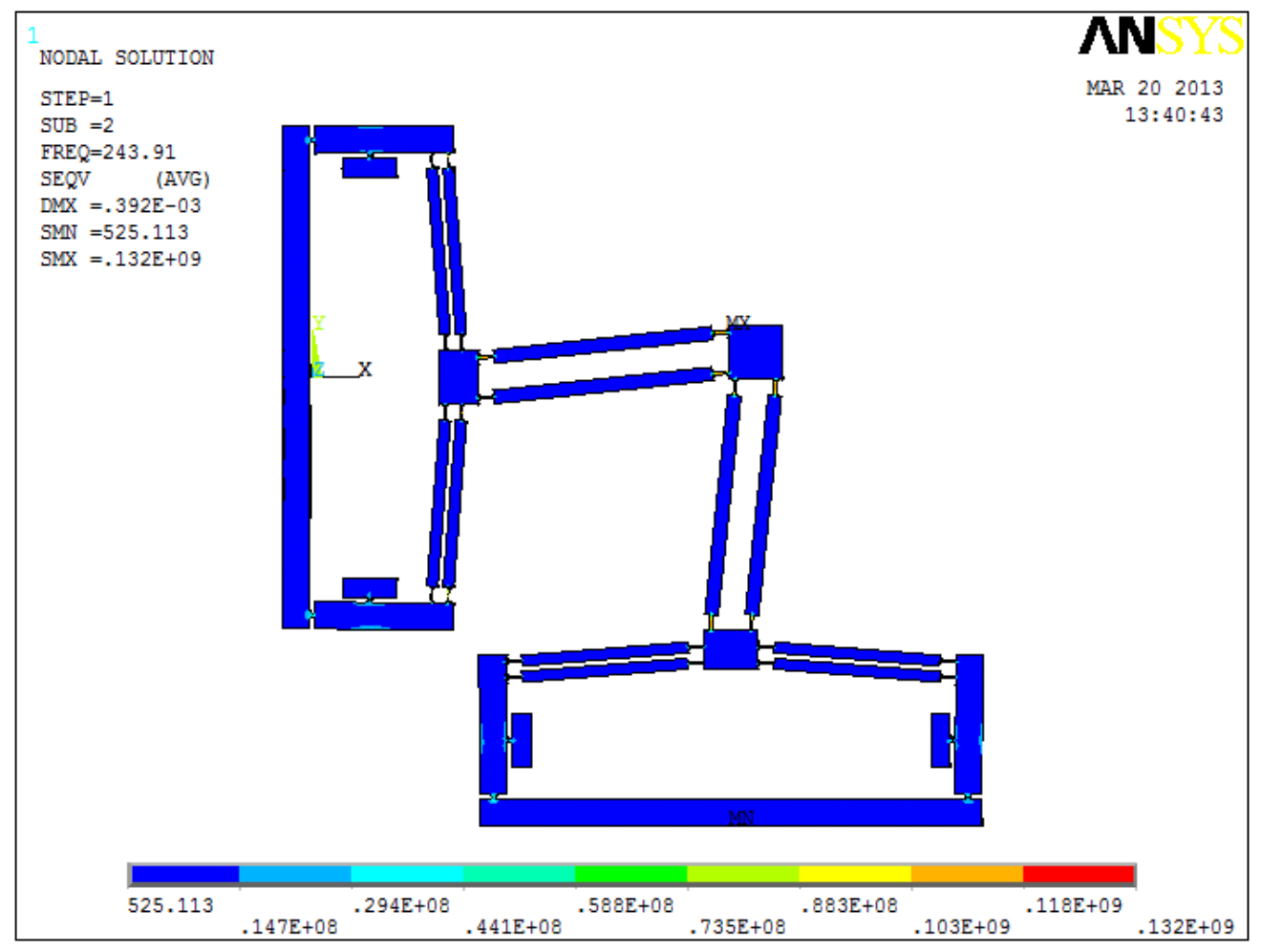

Figure 4.5 Contour plot of the Von-Mises stress of the optimized CMDA 
From the optimized results, it can be observed that the overall AR of the device increased from 32.8 to 39.2 while the NF decreased from 293.09 [ $\mathrm{Hz}]$ for the initial design to 243.91 $[\mathrm{Hz}]$ for the optimized design. Such a result is expected as the goal of the optimization is to achieve high AR value with a small sacrifice of NR. Figure 4.6 shows the corresponding mode shape to the found NF for the first case. Similar results have also been obtained for cases 2 to 4 . Comparing Table 4.6 with Table 4.3, one can see that the ARs are larger and the NRs are less for the optimized designs. All these results are expected as the objective function for the optimization is the maximum amplification ratio in the design. Figure 4.7 also shows the mode shape corresponding to the second case where displacements of 10 [ $\mu \mathrm{m}]$ in the $X$ direction were applied to the Leg A of the device. 

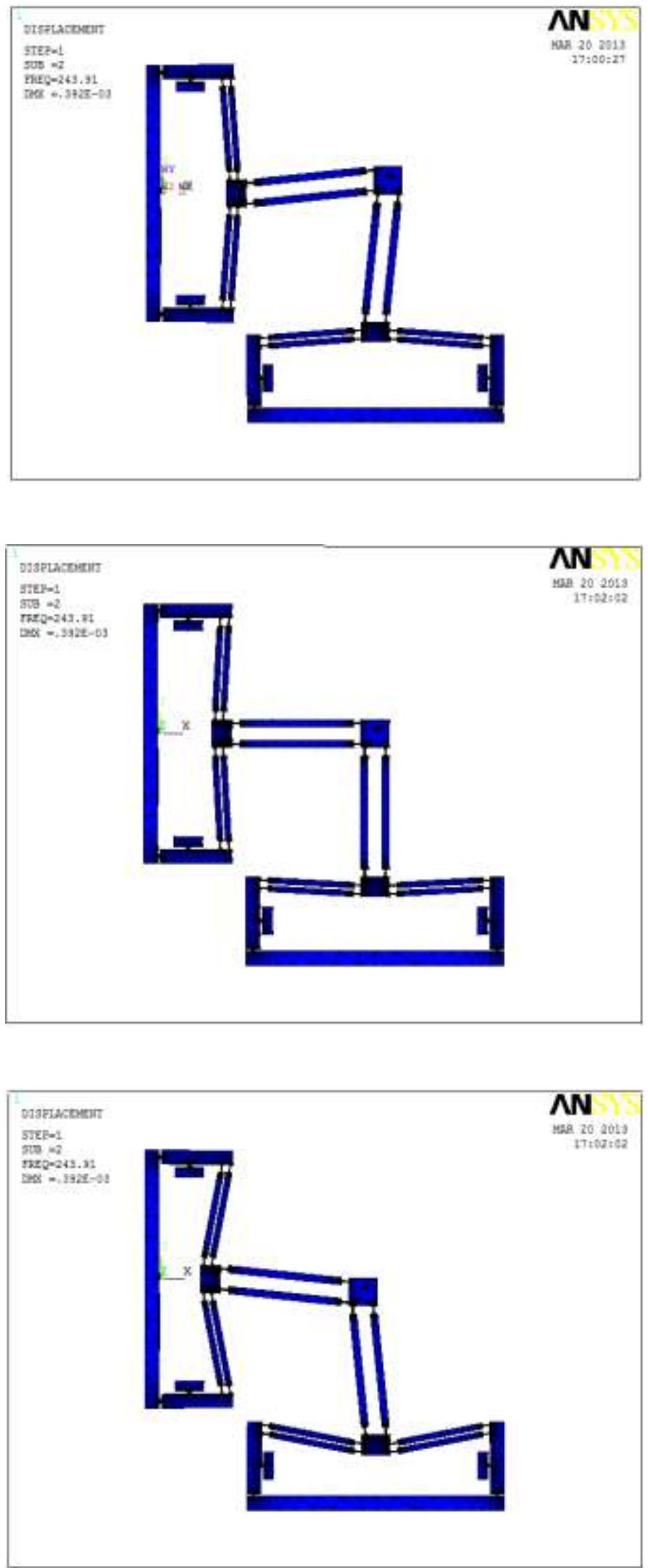

Figure 4.6 Mode shape of the device corresponding to the first case. 

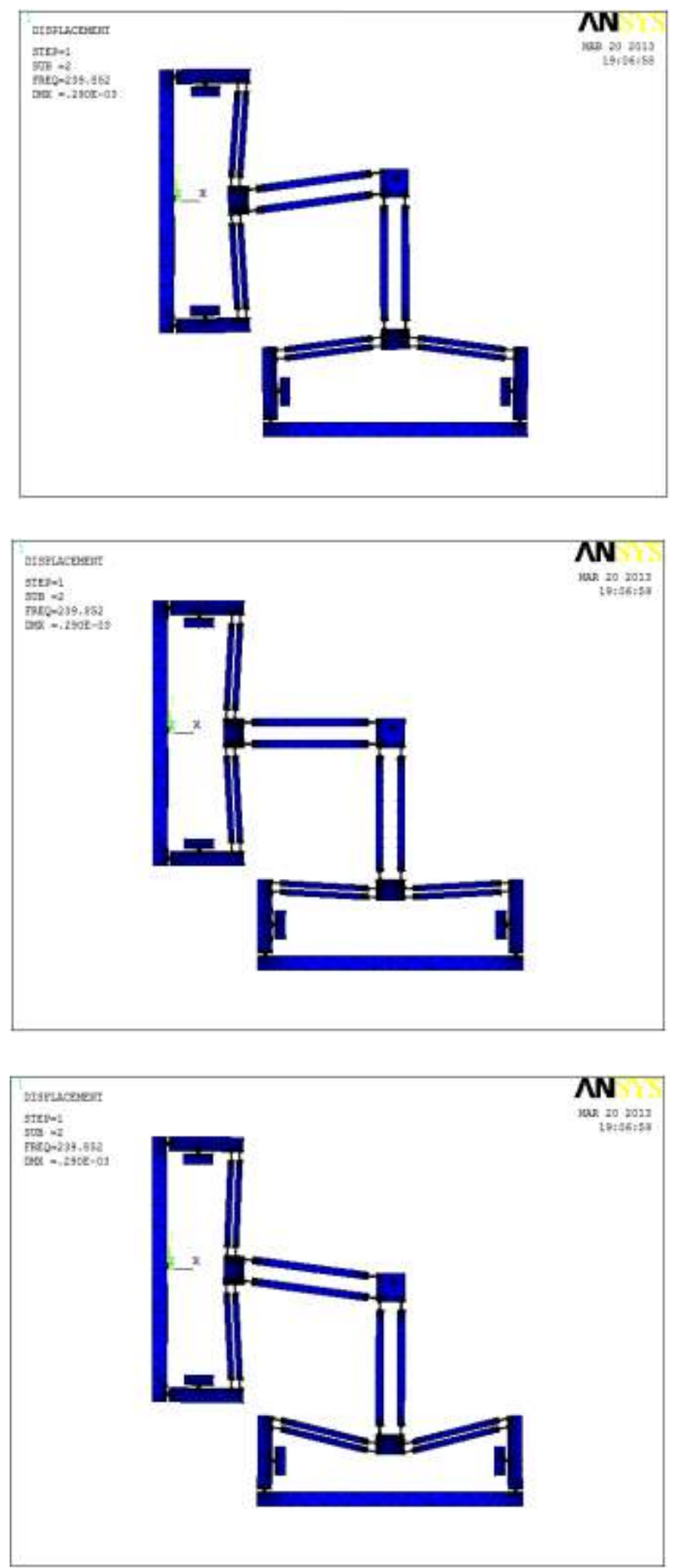

Figure 4.7 Mode shape of the device corresponding to the second case 


\subsubsection{Fabricated CDMA}

A full scale model of the proposed device (Figure 4.8) based on the optimized results is manufactured and will be tested in future to compare the obtained FEM results to that obtained by real experiments.

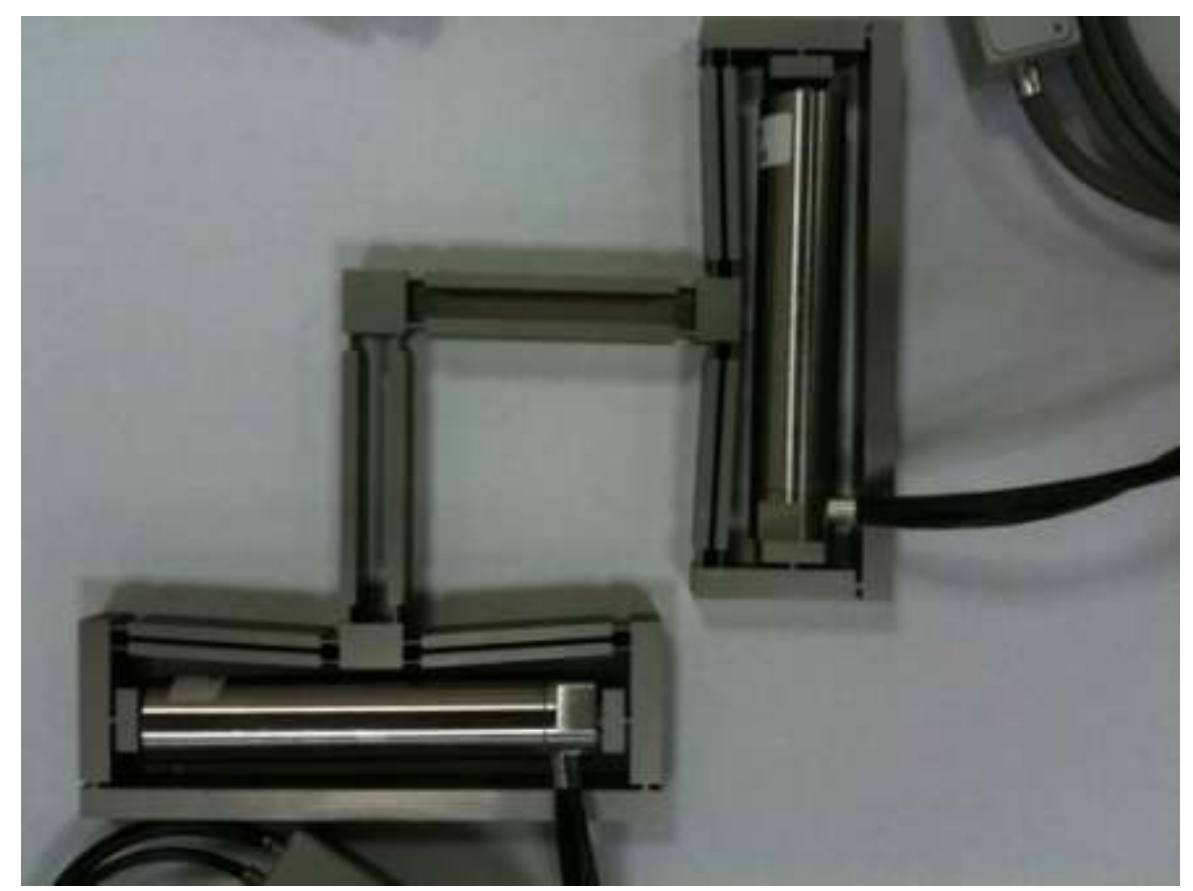

Figure 4.8 Manufactured XY planar motion CDMA integrated with two PZT actuators

It should be mentioned that All the ANSYS® codes used for designing, analysing, and optimizing of the proposed XY planar motion generator CMDA are provided in Appendix C. A CATIA ${ }^{\circledR}$ drawing of the design optimized mechanism outlining all the actual dements of the device is also included in Appendix D. 


\subsection{Conclusion}

In this chapter, the design, modeling, and optimization of a planar motion generator CMDA based on the design of a symmetric five-bar CMDA are presented. The goal of optimization process is to achieve high amplification ratio for the device and have a relative high natural frequency. The planar motion generator CMDA is capable of converting and amplifying the linear motion of PZT actuator to a large range planar (2-D) output motion. Some important parameters are identified and the optimized values are obtained for the purpose of the maximum output displacement of the designed CMDA for a selected specified PZT actuator. Detailed analysis with finite element method of static and dynamic characteristics of the proposed $\mathrm{XY}$ CMDA design is also provided. Through the optimization process, a $\mathrm{XY}$ compliant mechanical displacement amplifier with high AR compared to the initial suboptimal design is provided.

In order to minimize the cross talk effect of the mechanism and decouple the $\mathrm{X}$ and $\mathrm{Y}$ motions of the device, the proposed mechanism can be design to have two extra legs and be fully symmetric along the $\mathrm{X}$ and $\mathrm{Y}$ axis. This however, will increase the cost of manufacturing and requires two more PZT actuator for the operation of the mechanism. 


\section{CONCLUSIONS AND FUTURE WORK}

\subsection{Overview and Conclusions}

This thesis study was aimed to develop two compliant based amplification mechanisms for displacement amplification purposes and precision positioning applications. The topology of each developed mechanisms were based on the symmetric five-bar structure (Ouyang, Zhang, \& Gupta, 2008) due to the advantages such as high AR and minimal loss of NF facilitated by this structure.

This thesis study was started with an extensive review on various topologies available for developing compliant based mechanical amplifiers (CMA). The potentials and drawbacks associated with each topology were then identified. Different optimization techniques for optimal performance of a developed CMA mechanism were also discussed and it was recognized that optimization approach of a CMA mechanism depends on the purpose, objectives, and tasks that is supposed to be achieved by the mechanism.

The first mechanism was developed to amplify the linear output displacement of a $P I C A^{\mathrm{TM}}$ $P-010.80 P$ PZT actuator with the consideration of the NF of the mechanism. The designed CMDA, in conjunction with the mentioned PZT actuator have applications in Magnetic Resonance Elastography for identifying the location of cancerous tissues in males prostate. The dynamic and static performance of the proposed mechanism was found using FEM analysis. Then, based on the given requirements, an optimization method was developed to maximize the devices optimal performance. Finally, a full scale model of the optimized 
mechanism was manufactured out of brass and tested successfully on mock up of a prostate using a MRI unit.

The second mechanism was developed to meet the requirement of many industrial applications of high precision manipulation systems which is capable of generating planar or special motions in the desired direction. The objective of the developed mechanism was to design a XY planar CMDA with displacement amplification and high motion resolution in each direction. Detailed FEM analysis results of the static and the dynamic characteristics of the proposed XY CMDA design were provided and an optimization process was developed to increase the Amplification Ratio (AR) of the proposed XY compliant mechanism with minimal compromise in NF.

\subsection{Major Contributions of the Thesis}

This research study proved that compliant mechanical amplifiers are the most rescannable choice of amplifying the displacement amplification purposes. The two developed mechanisms developed in this study also revealed the potential of the symmetric five-bar structure and its superiority over other available topologies for the design of compliant based mechanical amplifiers. It was shown that with appropriate modifications, the symmetric fivebar topology is very versatile of meeting different industrial or pharmaceutical requirements and can find applications in many related fields.

\subsection{Future Research Work}

The following points can be recommending for future research work of the two developed mechanisms: 
First, although the design, developed, FEM analysis, and MRI testing of the CMDA for the use in prostate MRE application was proved to be successful, more precise experiments are required to get the actual dynamic and static response of the design mechanism to verify the results obtained by the FEM analysis.

Second, in the case of the developed XY planar motion generator CMDA, experiments should be performed to validate the FEM results and obtain the actual performance characteristics of the developed mechanism.

Third, in the case of the XY planar motion generator CMDA also, precision analysis should be performed in order to find the actual resolution and accuracy of the generated motion at the device's output. 


\section{REFERENCES}

ANSYS. (2009). ANSYS HELP. SAS IP.

Arani, A., Plewes, D., Krieger, A., \& Chopra, R. (2011). The Feasibility of Endorectal MR Elastography for Prostate Cancer Localization. Magnetic Resonance in Medicine , 66, 16491657.

Bharti, S., \& Frecker, M. (November 15-21, 2003). COMPLIANT MECHANICAL AMPLIFIER DESIGN USING MULTIPLE OPTIMALLY PLACED ACTUATORS. 2003 ASME International Mechanical Engineering Congress, (pp. 1-8). Washington, D.C.

Choia, K.-B., Lee, J. J., \& Hata, S. (2010). A piezo-driven compliant stage with double mechanical amplificationmechanisms arranged in parallel. Sensors and Actuators A: Physical , 167 (1), 173-181.

Dinesh, M., \& Ananthasuresh, G. K. ( December 12-13, 2007). A Topology-Optimized LargeRange Compliant X-Y Micro Stage. 13th National Conference on Mechanisms and Machines, (pp. 97-104). Bangalore, India.

Dunning, A. G., Tolou, N., \& Herder, J. L. (2013). A compact low-stiffness six degrees of freedom compliant precision stage. Precision Engineering , 37 (2), 380-388.

Furukawa, E., Mizuno, M., \& and Doi, T. (1995). Development of a Flexure-Hinged Translation Mechanism Driven by Two Piezoelectric Stacks. Int. J., Ser. C, , 38 (4), 743-748.

Hou, W. F., \& Zhang, X. M. (2009). Dynamic analysis of flexible linkage mechanism under uniform temperature change. Journal of Sound and Vibration, 319 (1-2), 570-592. 
Howell, L. L. (2001). Compliant Mechanisms. New York: Wiley and Sons.

Hua, W., \& Xianmin, Z. (2008). Input coupling analysis and optimal design of a 3-DOF compliant micro-positioning stage. Mechanism and Machine Theory, 43 (4), 400-410.

Hull, P. V., \& Canfield, S. (2006). Optimal Synthesis of Compliant Mechanisms Using Subdivision and Commercial FEA. Mech. Des. , 128 (2), 337-348.

Jiang, J., \& Mockensturm, E. M. (2003). A Novel Motion Amplifier Using Axially Driven Buckling Beam. Proceedings of the 2003 ASME Internationa. 16-21. Washington, DC,: ASME.

Kota, S., Hetrick, J., Li, Z., \& Saggere, L. (1999). Tailoring Unconventional Actuators Using Compliant Transmissions: Design Methods and Applications. Trans. Mechatron, 396-408.

Li, Y., \& Xu, Q. (2011). A Totally Decoupled Piezo-Driven XYZ Flexure Parallel Micropositioning Stage for Micro/Nanomanipulation. IEEE TRANSACTIONS ON AUTOMATION SCIENCE AND ENGINEERING , 8 (2), 265-279.

Li, Y., \& Xu, Q. (2011). Analytical modeling, optimization and testing of a compound bridgetype compliant displacement amplifier. Mechanism and Machine Theory, 46 (2), 183-200.

Loberto G. A., M. A. (2004). Development of a XY Piezoelectric Nanopositioner. ABCM Symposium Series In Mechatronics , 1, 662-671.

Lobontiu, N. (2002). Compliant Mechanisms, Dsign of Flexural Hinges . In N. Lobontiu, Compliant Mechanisms, Dsign of Flexural Hinges . Newyork: CRC Press LLC.

Lobontiu, N., Paine, J. S., Garcia, E., \& Goldfarb, M. (2001). Corner Filleted Flexure Hinges,. ASME J. Mech. Des. , 123 (3), 346-352. 
Lu, K. J., \& Kota, S. (2006). Topology and Dimensional Synthesis of Compliant. Mech. Des , 1080-1091.

Manoach, E., \& Ribeiro, P. (2004). Coupled, thermoelastic, large amplitude vibrations of Timoshenko beams. International Journal of Mechanical Sciences , 46 (11), 1589-606.

Marler, R. T., \& Arora, J. S. (2006). Survey of Multi-objective Optimization Methods for Engineering,". Structures and Multidisciplinary Optimization , 26, 369-395.

Ouyang, P. R. (2011). A Spatial Hybrid Motion Mechanism: Design and Optimization. Mechatronics , 21 (3), 479-489.

Ouyang, P. R., Tjiptoprodjo, R. C., Zhang, W. J., \& Yang, G. S. (2008). Micro-motion devices technology: The state of arts review. Int J Adv Manuf Technol , 463-478.

Ouyang, P. R., Zhang, W. J., \& Gupta, M. M. (2008). A New Compliant Mechanical Amplifier Based on a Symmetric Five-Bar Topology. Journal of Mechanical Design, 130 (10).

Palli, G., \& Pirozzi, S. (2011). Force sensor based on discrete optoelectronic components and compliant frames. Sensors and Actuators A: Physical , 165 (2), 39-249.

Park, S. R., \& Yang, S. H. (2005). A mathematical approach for analyzing ultra precision positioning system with compliant mechanism. Journal of Materials Processing Technology, $164-165,1584-1589$.

Pedersen, C. B., \& Seshia, A. A. (2004). On the optimization of compliant force amplifier mechanisms for surface micromachined resonant accelerometers. JOURNAL OF MICROMECHANICS AND MICROENGINEERING , 14 (10), 1281-1293. 
Pokines, B. J., \& Garcia, E. (1998). A Smart Material Microamplification Mechanism Fabricated Using LIGA. Smart Mater. Struct, 105-112.

Polit, S., \& Dong, J. (2011). Development of a High-Bandwidth XY Nanopositioning Stage for High-Rate Micro-/Nanomanufacturing. IEEE/ASME TRANSACTIONS ON MECHATRONICS , $16(4), 724-733$.

Przemieniecki, J. S. (1976). Theory of matrix structural analysis. New York: McGraw-Hill.

Rue, J. W., \& Gweon, D. G. (1997). Error Analysis of a Flexure Hinge Mechanism Induced by Machining Imperfection. Precis. Eng , 21 (1), 83-89.

Saif, M. T., \& MacDonald, N. C. (1998). Measurement of Forces and Spring Constants of Microinstruments. Rev. Sci. Instrum , 69 (3), 1410-1422.

Shih, C. J., \& Chen, K. Y. (01 - 05 June 2008). Optimum Topological Design for Mechanical Amplifying Mechanism Integrated in PZT Nano-Positioning Stage. EngOpt 2008 - International Conference on Engineering Optimization, (pp. 1-8). Rio de Janeiro, Brazil.

Shih, C. J., \& Lin, C. F. (2006). A two-stage topological optimum design for monolithic compliant microgripper integrated with flexure hinges. International MEMS Conference 2006, 34 , pp. 840-846.

Su, H. J., \& McCathy, J. M. (2007). Synthesis of Bistable Compliant Four-bar Mechanisms using Polynomial Homotopy. Mech. Des. , 1094-1098.

Su, X.-P. S., \& Henry, Y. S. (2001). Design of compliant microleverage mechanisms. Sensors and Actuators , 87 (3), 146-156. 
Sun, Z. H., Zhang, B., Cheng, L., \& Zhang, W. J. (2011). Application of the Redundant Servomotor Approach to Design of Path Generator with Dynamic Performance Improvement. Mechanism and Machine Theory. Mechanism and Machine Theory, 21 (3), 1784-1795.

Tian, Y., Shirinzadeh, B., Zhang, D., \& Zhong, Y. (2010). Three flexure hinges for compliant mechanism designs based on dimensionless graph analysis. Precision Engineering , 34 (1), $92-$ 100.

Timoshenko, S. P., \& and Gere, J. M. (1961). Theory of Elastic Stability. New York.: McGrawHill.

Ü. Sönmez, C. T. (2008). Compliant bistable mechanism design incorporating Elastica buckling beam theory and pseudo-rigid-body model. ASME J. Mech. Des , 130 (4), 42304-42318.

Xu, Q. (2012). Design and Development of a Flexure-Based Dual-Stage Nanopositioning System With Minimum Interference Behavior. IEEE TRANSACTIONS ON AUTOMATION SCIENCE AND ENGINEERING , 9 (3), 554 - 563.

Xu, W., \& King, T. (1996). piezoactuator displacement amplifiers: flexibility, accuracy, and stress considerations. Precision Engineering , 19 (2), 4-10.

Yang, R. Y., Jouaneh, M., \& Scheweizer, R. (1996). Design and Characterization of a LowProfile Micropositioning Stage. Precis. Eng. , 20-29.

Yong, Y. K., \& Lu, T.-F. (2009). Kinetostatic modeling of 3-RRR compliant micro-motion stages with flexure hinges. Mechanism and Machine Theory, 38 (6), 1156-1175.

Zhang, X., \& Hou, W. (2010). Dynamic analysis of the precision compliant mechanisms considering thermal effect. Precision Engineering , 34 (3), 592-606. 


\section{APPENDIX A}

\section{Unloaded CMDA}

\begin{tabular}{|c|c|c|c|}
\hline \multicolumn{4}{|c|}{ Original Design } \\
\hline Parameter in ANSYS & Optimized Values [m] & Amplification Ratio & $\begin{array}{c}\text { Resonant Frequency } \\
{[\mathrm{Hz}]}\end{array}$ \\
\hline H1 & 0.008318 & \multirow{6}{*}{7.35} & \multirow{6}{*}{475.54} \\
\hline HB_T & 0.006 & & \\
\hline HB & 0.006 & & \\
\hline $\mathbf{T 2}$ & 0.0008 & & \\
\hline R1 & 0.029753 & & \\
\hline S_ARMS & 0.025 & & \\
\hline Objective Function & \multicolumn{3}{|c|}{$100-\left[\frac{0.9 * D M A X}{140 E-6}+\frac{0.1 * F R E Q 1}{500}\right]$} \\
\hline Parameter in ANSYS & Optimized Values [m] & Amplification Ratio & $\begin{array}{c}\text { Resonant Frequency } \\
{[\mathrm{Hz}]}\end{array}$ \\
\hline H1 & $0.84815 \mathrm{E}-02$ & \multirow{6}{*}{8.6} & \multirow{6}{*}{340.002} \\
\hline HB_T & $0.60609 \mathrm{E}-02$ & & \\
\hline HB & $0.50515 \mathrm{E}-02$ & & \\
\hline T2 & $0.83544 \mathrm{E}-03$ & & \\
\hline R1 & $0.42518 \mathrm{E}-01$ & & \\
\hline S_ARMS & $0.19774 \mathrm{E}-01$ & & \\
\hline Objective Function & \multicolumn{3}{|c|}{$100-\left[\frac{0.8 * D M A X}{140 E-6}+\frac{0.2 * F R E Q 1}{500}\right]$} \\
\hline Parameter in ANSYS & Optimized Values [m] & Amplification Ratio & $\begin{array}{c}\text { Resonant Frequency } \\
{[\mathrm{Hz}]}\end{array}$ \\
\hline H1 & $0.83180 \mathrm{E}-02$ & \multirow{6}{*}{9.6} & \multirow{6}{*}{384.071} \\
\hline HB_T & $0.53289 \mathrm{E}-02$ & & \\
\hline$\overline{\mathrm{HB}}$ & $0.47894 \mathrm{E}-02$ & & \\
\hline T2 & $0.80000 \mathrm{E}-03$ & & \\
\hline R1 & $0.29753 \mathrm{E}-01$ & & \\
\hline S_ARMS & $0.15000 \mathrm{E}-01$ & & \\
\hline Objective Function & \multicolumn{3}{|c|}{$100-\left[\frac{0.7 * D M A X}{140 E-6}+\frac{0.3 * F R E Q 1}{500}\right]$} \\
\hline Parameter in ANSYS & Optimized Values [m] & Amplification Ratio & $\begin{array}{c}\text { Resonant Frequency } \\
{[\mathrm{Hz}]}\end{array}$ \\
\hline H1 & $0.83180 \mathrm{E}-02$ & \multirow{6}{*}{9.4} & \multirow{6}{*}{385.468} \\
\hline HB_T & $0.56411 \mathrm{E}-02$ & & \\
\hline HB & $0.58834 \mathrm{E}-02$ & & \\
\hline $\mathbf{T 2}$ & $0.80000 \mathrm{E}-03$ & & \\
\hline R1 & $0.30613 \mathrm{E}-01$ & & \\
\hline S_ARMS & $0.15000 \mathrm{E}-01$ & & \\
\hline Objective Function & \multicolumn{3}{|c|}{$100-\left[\frac{0.6 * D M A X}{140 E-6}+\frac{0.4 * F R E Q 1}{500}\right]$} \\
\hline
\end{tabular}




\begin{tabular}{|c|c|c|c|}
\hline Parameter in ANSYS & Optimized Values [m] & Amplification Ratio & $\begin{array}{c}\text { Resonant Frequency } \\
{[\mathrm{Hz}]}\end{array}$ \\
\hline H1 & $0.85313 \mathrm{E}-02$ & \multirow{6}{*}{11.85} & \multirow{6}{*}{318.285} \\
\hline HB_T & $0.40000 \mathrm{E}-02$ & & \\
\hline $\mathbf{H B}$ & $0.60052 \mathrm{E}-02$ & & \\
\hline $\mathbf{T 2}$ & $0.80000 \mathrm{E}-03$ & & \\
\hline R1 & $0.45000 \mathrm{E}-01$ & & \\
\hline S_ARMS & $0.15000 \mathrm{E}-01$ & & \\
\hline Objective Function & \multicolumn{3}{|c|}{$100-\left[\frac{0.5 * D M A X}{140 E-6}+\frac{0.5 * F R E Q 1}{500}\right]$} \\
\hline Parameter in ANSYS & Optimized Values [m] & Amplification Ratio & $\begin{array}{c}\text { Resonant Frequency } \\
{[\mathrm{Hz}]}\end{array}$ \\
\hline H1 & $0.86146 \mathrm{E}-02$ & \multirow{6}{*}{11.85} & \multirow{6}{*}{315.072} \\
\hline HB_T & $0.40000 \mathrm{E}-02$ & & \\
\hline $\mathbf{H B}$ & $0.55522 \mathrm{E}-02$ & & \\
\hline T2 & $0.80000 \mathrm{E}-03$ & & \\
\hline R1 & $0.45000 \mathrm{E}-01$ & & \\
\hline S_ARMS & $0.15000 \mathrm{E}-01$ & & \\
\hline Objective Function & \multicolumn{3}{|c|}{$100-\left[\frac{0.4 * D M A X}{140 E-6}+\frac{0.6 * F R E Q 1}{500}\right]$} \\
\hline Parameter in ANSYS & Optimized Values & Amplification Ratio & Resonant Frequency \\
\hline H1 & $0.83283 \mathrm{E}-02$ & \multirow[t]{6}{*}{ T } & \multirow{6}{*}{316.102} \\
\hline HB_T & $0.40000 \mathrm{E}-02$ & & \\
\hline HB & $0.48790 \mathrm{E}-02$ & & \\
\hline $\mathbf{T 2}$ & $0.80000 \mathrm{E}-03$ & & \\
\hline R1 & $0.41763 \mathrm{E}-01$ & & \\
\hline S_ARMS & $0.15000 \mathrm{E}-01$ & & \\
\hline Objective Function & \multicolumn{3}{|c|}{$100-\left[\frac{0.3 * D M A X}{140 E-6}+\frac{0.7 * F R E Q 1}{500}\right]$} \\
\hline Parameter in ANSYS & Optimized Values [m] & Amplification Ratio & $\begin{array}{c}\text { Resonant Frequency } \\
{[\mathrm{Hz}]}\end{array}$ \\
\hline H1 & $0.83180 \mathrm{E}-02$ & \multirow{6}{*}{11.65} & \multirow{6}{*}{306.197} \\
\hline HB_T & $0.40000 \mathrm{E}-02$ & & \\
\hline HB & $0.48474 \mathrm{E}-02$ & & \\
\hline T2 & $0.80000 \mathrm{E}-03$ & & \\
\hline R1 & $0.44062 \mathrm{E}-01$ & & \\
\hline S_ARMS & $0.15000 \mathrm{E}-01$ & & \\
\hline Objective Function & \multicolumn{3}{|c|}{$100-\left[\frac{0.2 * D M A X}{140 E-6}+\frac{0.8 * F R E Q 1}{500}\right]$} \\
\hline Parameter in ANSYS & Optimized Values [m] & Amplification Ratio & $\begin{array}{c}\text { Resonant Frequency } \\
{[\mathrm{Hz}]}\end{array}$ \\
\hline H1 & $0.83180 \mathrm{E}-02$ & \multirow{4}{*}{11.25} & \multirow{4}{*}{294.08} \\
\hline HB_T & $0.40000 \mathrm{E}-02$ & & \\
\hline $\mathbf{H B}$ & $0.42796 \mathrm{E}-02$ & & \\
\hline $\mathbf{T 2}$ & $0.80000 \mathrm{E}-03$ & & \\
\hline
\end{tabular}




\begin{tabular}{|c|c|c|c|}
\hline R1 & $0.45000 \mathrm{E}-01$ & \\
\hline S_ARMS & $0.15000 \mathrm{E}-01$ & \\
\cline { 1 - 2 } Objective Function & \multicolumn{2}{|c|}{$100-\left[\frac{0.1 * D M A X}{140 E-6}+\frac{0.9 * F R E Q 1}{500}\right]$} \\
\hline Parameter in ANSYS & Optimized Values [m] & \multirow{2}{*}{ Amplification Ratio } & $\begin{array}{c}\text { Resonant Frequency } \\
\text { [Hz] }\end{array}$ \\
\hline H1 & $0.86296 \mathrm{E}-02$ & & \\
\hline HB_T & $0.73572 \mathrm{E}-02$ & & \\
\hline HB & $0.72624 \mathrm{E}-02$ & \multirow{2}{*}{8.9} & \\
\hline T2 & $0.80000 \mathrm{E}-03$ & & \\
\hline S1 & $0.45000 \mathrm{E}-01$ & & \\
\hline S_ARMS & $0.15000 \mathrm{E}-01$ & & \\
\hline
\end{tabular}

\section{Loaded CMDA With 274.61 g Mass}

\begin{tabular}{|c|c|c|c|}
\hline \multicolumn{4}{|c|}{ Original Design } \\
\hline Parameter in ANSYS & Optimized Values [m] & Amplification Ratio & $\begin{array}{c}\text { Resonant Frequency } \\
{[\mathrm{Hz}]}\end{array}$ \\
\hline H1 & 0.008318 & \multirow{6}{*}{7.65} & \multirow{6}{*}{188.051} \\
\hline HB_T & 0.006 & & \\
\hline HB & 0.006 & & \\
\hline T2 & 0.0008 & & \\
\hline R1 & 0.029753 & & \\
\hline S_ARMS & 0.025 & & \\
\hline Objective Function & \multicolumn{3}{|c|}{$100-\left[\frac{0.9 * D M A X}{140 E-6}+\frac{0.1 * F R E Q 1}{500}\right]$} \\
\hline Parameter in ANSYS & Optimized Values [m] & Amplification Ratio & $\begin{array}{c}\text { Resonant Frequency } \\
{[\mathrm{Hz}]}\end{array}$ \\
\hline H1 & $0.84815 \mathrm{E}-02$ & \multirow{6}{*}{9.15} & \multirow{6}{*}{135.391} \\
\hline HB_T & 0.60609E-02 & & \\
\hline HB & $0.50515 \mathrm{E}-02$ & & \\
\hline T2 & $0.83544 \mathrm{E}-03$ & & \\
\hline R1 & $0.42518 \mathrm{E}-01$ & & \\
\hline S_ARMS & $0.19774 \mathrm{E}-01$ & & \\
\hline Objective Function & \multicolumn{3}{|c|}{$100-\left[\frac{0.8 * D M A X}{140 E-6}+\frac{0.2 * F R E Q 1}{500}\right]$} \\
\hline Parameter in ANSYS & Optimized Values [m] & Amplification Ratio & $\begin{array}{c}\text { Resonant Frequency } \\
{[\mathrm{Hz}]} \\
\end{array}$ \\
\hline H1 & $0.83180 \mathrm{E}-02$ & \multirow{6}{*}{10.05} & \multirow{6}{*}{147.79} \\
\hline HB_T & $0.53289 \mathrm{E}-02$ & & \\
\hline HB & $0.47894 \mathrm{E}-02$ & & \\
\hline T2 & $0.80000 \mathrm{E}-03$ & & \\
\hline R1 & $0.29753 \mathrm{E}-01$ & & \\
\hline S_ARMS & $0.15000 \mathrm{E}-01$ & & \\
\hline Objective Function & \multicolumn{3}{|c|}{$100-\left[\frac{0.7 * D M A X}{140 E-6}+\frac{0.3 * F R E Q 1}{500}\right]$} \\
\hline
\end{tabular}




\begin{tabular}{|c|c|c|c|}
\hline Parameter in ANSYS & Optimized Values [m] & Amplification Ratio & $\begin{array}{c}\text { Resonant Frequency } \\
{[\mathrm{Hz}]}\end{array}$ \\
\hline H1 & $0.83180 \mathrm{E}-02$ & \multirow{6}{*}{9.85} & \multirow{6}{*}{151.289} \\
\hline HB_T & $0.56411 \mathrm{E}-02$ & & \\
\hline HB & $0.58834 \mathrm{E}-02$ & & \\
\hline $\mathbf{T 2}$ & $0.80000 \mathrm{E}-03$ & & \\
\hline R1 & $0.30613 \mathrm{E}-01$ & & \\
\hline S_ARMS & $0.15000 \mathrm{E}-01$ & & \\
\hline Objective Function & \multicolumn{3}{|c|}{$100-\left[\frac{0.6 * D M A X}{140 E-6}+\frac{0.4 * F R E Q 1}{500}\right]$} \\
\hline Parameter in ANSYS & Optimized Values [m] & Amplification Ratio & $\begin{array}{c}\text { Resonant Frequency } \\
{[\mathrm{Hz}]}\end{array}$ \\
\hline H1 & $0.85313 \mathrm{E}-02$ & \multirow{6}{*}{12.75} & \multirow{6}{*}{109.684} \\
\hline HB_T & $0.40000 \mathrm{E}-02$ & & \\
\hline $\mathbf{H B}$ & $0.60052 \mathrm{E}-02$ & & \\
\hline T2 & $0.80000 \mathrm{E}-03$ & & \\
\hline R1 & $0.45000 \mathrm{E}-01$ & & \\
\hline S_ARMS & $0.15000 \mathrm{E}-01$ & & \\
\hline Objective Function & \multicolumn{3}{|c|}{$100-\left[\frac{0.5 * D M A X}{140 E-6}+\frac{0.5 * F R E Q 1}{500}\right]$} \\
\hline Parameter in ANSYS & Optimized Values [m] & Amplification Ratio & $\begin{array}{c}\text { Resonant Frequency } \\
{[\mathrm{Hz}]}\end{array}$ \\
\hline H1 & $0.86146 \mathrm{E}-02$ & \multirow{6}{*}{12.75} & \multirow{6}{*}{108.685} \\
\hline HB_T & $0.40000 \mathrm{E}-02$ & & \\
\hline HB & $0.55522 \mathrm{E}-02$ & & \\
\hline T2 & $0.80000 \mathrm{E}-03$ & & \\
\hline $\mathbf{R 1}$ & $0.45000 \mathrm{E}-01$ & & \\
\hline S_ARMS & $0.15000 \mathrm{E}-01$ & & \\
\hline Objective Function & \multicolumn{3}{|c|}{$100-\left[\frac{0.4 * D M A X}{140 E-6}+\frac{0.6 * F R E Q 1}{500}\right]$} \\
\hline Parameter in ANSYS & Optimized Values & Amplification Ratio & Resonant Frequency \\
\hline H1 & $0.83283 \mathrm{E}-02$ & \multirow{6}{*}{12.6} & \multirow{6}{*}{109.049} \\
\hline HB_T & $0.40000 \mathrm{E}-02$ & & \\
\hline HB & $0.48790 \mathrm{E}-02$ & & \\
\hline T2 & $0.80000 \mathrm{E}-03$ & & \\
\hline R1 & $0.41763 \mathrm{E}-01$ & & \\
\hline S_ARMS & $0.15000 \mathrm{E}-01$ & & \\
\hline Objective Function & \multicolumn{3}{|c|}{$100-\left[\frac{0.3 * D M A X}{140 E-6}+\frac{0.7 * F R E Q 1}{500}\right]$} \\
\hline Parameter in ANSYS & Optimized Values [m] & Amplification Ratio & $\begin{array}{c}\text { Resonant Frequency } \\
{[\mathrm{Hz}]} \\
\end{array}$ \\
\hline H1 & $0.83180 \mathrm{E}-02$ & \multirow{4}{*}{12.65} & \multirow{4}{*}{105.239} \\
\hline HB_T & $0.40000 \mathrm{E}-02$ & & \\
\hline $\mathbf{H B}$ & $0.48474 \mathrm{E}-02$ & & \\
\hline $\mathbf{T 2}$ & $0.80000 \mathrm{E}-03$ & & \\
\hline
\end{tabular}




\begin{tabular}{|c|c|c|c|}
\hline R1 & $0.44062 \mathrm{E}-01$ & & \\
\hline S_ARMS & $0.15000 \mathrm{E}-01$ & & \\
\hline Objective Function & \multicolumn{3}{|c|}{$100-\left[\frac{0.2 * D M A X}{140 E-6}+\frac{0.8 * F R E Q 1}{500}\right]$} \\
\hline Parameter in ANSYS & Optimized Values [m] & Amplification Ratio & $\begin{array}{c}\text { Resonant Frequency } \\
{[\mathrm{Hz}]}\end{array}$ \\
\hline H1 & $0.83180 \mathrm{E}-02$ & \multirow{6}{*}{12.35} & \multirow{6}{*}{101.018} \\
\hline HB_T & $0.40000 \mathrm{E}-02$ & & \\
\hline$\overline{\mathbf{H B}}$ & $0.42796 \mathrm{E}-02$ & & \\
\hline T2 & $0.80000 \mathrm{E}-03$ & & \\
\hline $\mathbf{R 1}$ & $0.45000 \mathrm{E}-01$ & & \\
\hline S_ARMS & $0.15000 \mathrm{E}-01$ & & \\
\hline Objective Function & \multicolumn{3}{|c|}{$100-\left[\frac{0.1 * \square \square \square \square}{140 \square-6}+\frac{0.9 * \square \square \square \square 1}{500}\right]$} \\
\hline Parameter in ANSYS & Optimized Values [m] & Amplification Ratio & $\begin{array}{c}\text { Resonant Frequency } \\
{[\mathrm{Hz}]}\end{array}$ \\
\hline H1 & $0.86296 \mathrm{E}-02$ & \multirow{6}{*}{9.5} & \multirow{6}{*}{130.946} \\
\hline HB_T & $0.73572 \mathrm{E}-02$ & & \\
\hline$\overline{\mathrm{HB}}$ & $0.72624 \mathrm{E}-02$ & & \\
\hline T2 & $0.80000 \mathrm{E}-03$ & & \\
\hline R1 & $0.45000 \mathrm{E}-01$ & & \\
\hline S_ARMS & $0.15000 \mathrm{E}-01$ & & \\
\hline
\end{tabular}




\section{APPENDIX B}

\section{ANSYS Codes for the Unloaded CMDA}

$$
\begin{aligned}
& \mathrm{r}=0.000500 \\
& \mathrm{~h} 1=0.008318 \\
& \mathrm{hb} \_\mathrm{t}=0.006 \\
& \mathrm{hb}=0.006 \\
& \mathrm{t} 2=0.0008 \\
& \mathrm{r} 1=0.029753 \\
& \mathrm{~s} \_\mathrm{arms}=0.025 \\
& \mathrm{r} 3=0.062605+0.002395 \\
& \mathrm{t}=0.0050000 \\
& \mathrm{~h} 2=-\mathrm{hb}-\mathrm{r} \\
& \mathrm{rt}=\mathrm{r} 3+\mathrm{t} \\
& \mathrm{rh} 1=\mathrm{r} 1-\mathrm{h} 1 \\
& \mathrm{thickness}=0.0127000 \\
& \mathrm{rf}=0.0001 \\
& \mathrm{t} 1=0.003000 \\
& \mathrm{t} 3=0.001347 \\
& \mathrm{t} 4=\mathrm{abs}(\mathrm{h} 1) \\
& \mathrm{t} 5=0.003500 \\
& \mathrm{t} 6=\left(\mathrm{hb} \_\mathrm{t}-\mathrm{t} 2-\mathrm{t} 3\right) / 2 \\
& \mathrm{~h} 3=0.0045 \\
& \mathrm{~h} 4=0.008 \\
& \mathrm{f}=0.000250 \\
& \mathrm{rb}=0.000500 \\
& \mathrm{fb}=0.000500 \\
& \mathrm{~h} \_\mathrm{act}=0.02 \\
& \text { load_top }=0.064778009
\end{aligned}
$$


$\mathrm{dt}=\mathrm{h} 1 * \mathrm{t} 1 /\left(\mathrm{r} 3-\mathrm{s} \_\right.$arms-t-hb $)$

/prep7

mp,ex,1,97.2e9

mp,nuxy, $1,0.311$

mp,dens, $1,8934.20721$

BTOL, 1e-6

BLC4,0,h2-hb,r3+hb,2*hb

RECTNG,r3-hb,r3+hb,h2-hb,r1+hb_t

RECTNG,r3-hb-s_arms, r3-hb,r1-hb_t,r1+hb_t

RECTNG,0,t,r1-h1-hb_t,r1-h1+hb_t

A, $15,14,9,12$

AADD,all

k,21,r3-hb-s_arms,r1-hb_t+t6

$\mathrm{k}, 22, \mathrm{r} 3-\mathrm{hb}-\mathrm{s} \_a r m s-\mathrm{t} 1, \mathrm{r} 1-\mathrm{hb} \_\mathrm{t}+\mathrm{t} 6-\mathrm{dt}$

k,23,r3-hb-s_arms-t1,r1-hb_t-dt-0.001

A,9,21,22,23

k,24,r3-hb-s_arms,r1+hb_t-t6

k,25,r3-hb-s_arms-t1,r1+hb_t-t6-dt

k,26,r3-hb-s_arms-t1,r1+hb_t

A, $12,24,25,26$

$\mathrm{K}, 27, \mathrm{t}, \mathrm{r} 1-\mathrm{h} 1-\mathrm{hb} \_\mathrm{t}+\mathrm{t} 6$

$\mathrm{k}, 28, \mathrm{t}+\mathrm{t} 1, \mathrm{r} 1-\mathrm{h} 1-\mathrm{hb} \_\mathrm{t}+\mathrm{t} 6+\mathrm{dt}$

k,29,t+t1,r1-h1-hb_t

A, $14,27,28,29$

$\mathrm{K}, 30, \mathrm{t}, \mathrm{r} 1-\mathrm{h} 1+\mathrm{hb} \_\mathrm{t}-\mathrm{t} 6$

$\mathrm{k}, 31, \mathrm{t}+\mathrm{t} 1, \mathrm{r} 1-\mathrm{h} 1+\mathrm{hb} \_\mathrm{t}-\mathrm{t} 6+\mathrm{dt}$

$\mathrm{k}, 32, \mathrm{t}+\mathrm{t} 1, \mathrm{r} 1+\mathrm{hb} \_\mathrm{t}$

A, 15,30,31,32 
$\mathrm{k}, 33, \mathrm{t}, \mathrm{r} 1-\mathrm{h} 1+\mathrm{hb} \_\mathrm{t}-\mathrm{t} 2-\mathrm{t} 6$

$\mathrm{k}, 34, \mathrm{t}+\mathrm{t} 1, \mathrm{r} 1-\mathrm{h} 1+\mathrm{hb} \_\mathrm{t}-\mathrm{t} 2-\mathrm{t} 6+\mathrm{dt}$

$\mathrm{k}, 35, \mathrm{t}+\mathrm{t} 1, \mathrm{r} 1-\mathrm{h} 1-\mathrm{hb} \_\mathrm{t}+\mathrm{t} 2+\mathrm{t} 6+\mathrm{dt}$

$\mathrm{k}, 36, \mathrm{t}, \mathrm{r} 1-\mathrm{h} 1-\mathrm{hb} \_\mathrm{t}+\mathrm{t} 2+\mathrm{t} 6$

A,33,34,35,36

k,37,r3-hb-t1-s_arms,r1+hb_t-t2-t6-dt

$\mathrm{k}, 38, \mathrm{r3}-\mathrm{hb}-\mathrm{s} \_a r m s, \mathrm{r} 1+\mathrm{hb}$-t-t2-t6

k,39,r3-hb-s_arms,r1-hb_t+t2+t6

k,40,r3-hb-t1-s_arms,r1-hb_t+t2+t6-dt

A,37,38,39,40

k,41,r3-hb-t1-s_arms,r1-t3-dt

$\mathrm{k}, 42, \mathrm{r} 3-\mathrm{hb}-\mathrm{t} 1-\mathrm{s} \_a r m s, \mathrm{r} 1+\mathrm{t} 3-\mathrm{dt}$

$\mathrm{k}, 43, \mathrm{t}+\mathrm{t} 1, \mathrm{r} 1-\mathrm{h} 1+\mathrm{dt}+\mathrm{t} 3$

$\mathrm{k}, 44, \mathrm{t}+\mathrm{t} 1, \mathrm{r} 1-\mathrm{h} 1+\mathrm{dt}-\mathrm{t} 3$

A,41,42,43,44

ASBA,6,ALL

lfillt,28,29,rf,, lfillt,29,47,rf,,

lfillt,35,32,rf,, lfillt,32,50,rf,,

lfillt, 35,34,rf,, lfillt, 34,51,rf,,

lfillt, 14,25,rf,, lfillt,25,46,rf,,

!! Right side

lfillt,45,10,rf,, lfillt, 10,8,rf,,

lfillt,52,36,rf,, lfillt,36,37,rf,,

lfillt, 53,38,rf,, lfillt,38,37,rf,, 
lfillt,44,3,rf,

lfillt, $3,1, \mathrm{rf}$,

al, $5,6,11$

al,27,26,30

al, $42,40,54$

al,59,58,60

al, $17,12,18$

al,31,33,39

al, $55,56,57$

al,61,62,63

al,64,65,66

al, $70,71,72$

al, $76,77,78$

al, $82,83,84$

al, $85,86,87$

al, $79,80,81$

al, $73,74,75$

al,67,68,69

lfillt,9,24,rf, al, $88,89,90$

AADD, all

CYL4,r3-fb-r,0,r

RECTNG,r3-hb,r3-fb-r,-r,r,

CYL4,r3+fb+r, $0, r$

RECTNG,r3+r+fb,r3+hb,-r,r,

BLC5,r3-hb-t5/2,h3,t5,h4

CYL4, r3-hb-r,h3-f-r,r

RECTNG, r3-hb-2*r,r3-hb,h3-h4/2,h3-f-r,

CYL4, r3-hb-r,h3+f+r,r

RECTNG, r3-hb-2*r,r3-hb,h3+f+r,h3+h4/2,

AADD, 5,19

ASBA, 10,ALL 
RECTNG,0,0.0025,r1-h1+hb_t,r1-h1+hb_t+h_act

BLC4,0,h2-hb,r3+hb + 0.015,2*hb

AADD, all

ARSYM, $x$, all

AADD,ALL

LCOMB, 118, 117,0

LCOMB, 117,116,0

LCOMB, 116,162,0

LCOMB, 116,20,0

LCOMB, 20,57,0

LCOMB, 114,164,0

LCOMB, 126,127,0

LCOMB, 23,7,0

LCOMB, 2,161,0

ET,1,PLANE82

KEYOPT, $1,3,3$

KEYOPT, $1,5,0$

KEYOPT, $1,6,0$

$\mathrm{r}, 1,0.0127000$

SMRT, 1

MSHAPE, 0,2D

$\mathrm{CM}$, Y,AREA

ASEL, , , 2

CM,_Y1,AREA

CHKMSH,'AREA'

CMSEL,S,_Y

AMESH,_Y1

CMDELE,_Y

CMDELE,_Y1

CMDELE,_Y2

$d x=10 e-6$

DL, 104,,ux,-dx

DL,69, ux,dx 
DL,20,,ALL

ACEL, $0,-9.8$

FINISH

/SOL

solv

*GET,F1,NODE,69,RF,FX

*GET,F2,NODE, 1725,RF,FX

*GET,F3,NODE, 1726,RF,FX

*GET,F4,NODE, 1727,RF,FX

*GET,F5,NODE, 1728,RF,FX

*GET,F6,NODE, 1729, RF,FX

*GET,F7,NODE, 1730,RF,FX

*GET,F8,NODE, 1731,RF,FX

*GET,F9,NODE, 1732,RF,FX

*GET,F10,NODE, 1733,RF,FX

*GET,F11,NODE,70,RF,FX

$\mathrm{F} 30=\mathrm{F} 1+\mathrm{F} 2+\mathrm{F} 3+\mathrm{F} 4+\mathrm{F} 5+\mathrm{F} 6+\mathrm{F} 7+\mathrm{F} 8$

$\mathrm{F} 31=\mathrm{F} 9+\mathrm{F} 10+\mathrm{F} 11$

FTOTAL=F30+F31

ForceMax $=2300-(19 E 6) * d x$

Force $=$ ForceMax-FTOTAL

FINISH

/SOLUTION

ANTYPE, 2

FINISH

/PREP7

FINISH

/SOL

MODOPT,LANB, 1

EQSLV,SPAR

MXPAND $, 1,,, 0$

LUMPM, 0

PSTRES, 0

MODOPT,LANB, 1,0,0, ,OFF

/STATUS,SOLU

SOLVE 


\section{/POST1}

AVPRIN,0,0,

ETABLE,Stress,S,EQV

ESORT,ETAB,STRESS,0,1, , *GET,StressMax,SORT,,MAX

AVPRIN,0,0,

ETABLE,UY,U,Y

ESORT,ETAB,UY,0,1, , *GET,DMAX,SORT,,MAX

*GET,freq1,MODE, 1,FREQ

obj=100-((0.8*DMAX)/140e-6)+((0.2*FREQ1)/500)

/OPT

OPVAR,H1,DV,0.008318,0.0089, OPVAR,HB_T,DV,0.004,0.008,, OPVAR,HB,DV,0.004,0.008, OPVAR,T2,DV,0.0008,0.0014,, OPVAR,R1,DV,0.029753,0.045, OPVAR,S_ARMS,DV,0.015,0.045, OPVAR,dx,DV,1e-6,120e-6,, OPVAR,StressMax,SV,0,800e6,, OPVAR,Force,SV,1,2300,1,

OPVAR,obj,OBJ, , , ,

\section{ANSYS Codes for the Loaded CMDA}

$$
\begin{aligned}
& \mathrm{r}=0.000500 \\
& \mathrm{r} 1=0.029753 \\
& \mathrm{r} 3=0.062605+0.002395 \\
& \mathrm{t}=0.0050000 \\
& \mathrm{hb}=0.006 \\
& \mathrm{~h} 1=0.008318 \\
& \mathrm{~h} 2=-\mathrm{hb}-\mathrm{r} \\
& \mathrm{rt}=\mathrm{r} 3+\mathrm{t} \\
& \mathrm{rh} 1=\mathrm{r} 1-\mathrm{h} 1
\end{aligned}
$$




$$
\begin{aligned}
& \text { thickness }=0.0127000 \\
& \mathrm{rf}=0.0001 \\
& \mathrm{t} 1=0.003000 \\
& \mathrm{t} 2=0.0008 \\
& \mathrm{t} 3=0.001347 \\
& \mathrm{t} 4=\mathrm{abs}(\mathrm{h} 1) \\
& \mathrm{t} 5=0.003500 \\
& \mathrm{hb} \_\mathrm{t}=0.006 \\
& \mathrm{t} 6=\left(\mathrm{hb} \_\mathrm{t}-\mathrm{t} 2-\mathrm{t} 3\right) / 2 \\
& \mathrm{~h} 3=0.0045 \\
& \mathrm{~h} 4=0.008 \\
& \mathrm{f}=0.000250 \\
& \mathrm{rb}=0.000500 \\
& \mathrm{fb}=0.000500 \\
& \mathrm{~h} \_\mathrm{act}=0.02 \\
& \text { load_top }=0.064778009 \\
& \mathrm{~s} \_\mathrm{arms}=0.025 \\
& \mathrm{dt}=\mathrm{h} 1 * \mathrm{t} 1 /\left(\mathrm{r} 3-\mathrm{s} \_\mathrm{arms}-\mathrm{t}-\mathrm{hb}\right) \\
& / \mathrm{prep} 7
\end{aligned}
$$

mp,ex, $1,97.2 \mathrm{e} 9$

mp,nuxy, $1,0.311$

mp,dens, $1,8934.20721$

BTOL,1e-6

BLC4,0,h2-hb,r3+hb,2*hb

RECTNG,r3-hb,r3+hb,h2-hb,r1+hb_t

RECTNG,r3-hb-s_arms, r3-hb,r1-hb_t,r1+hb_t

RECTNG,0,t,r1-h1-hb_t,r1-h1+hb_t

$\mathrm{A}, 15,14,9,12$ 
AADD,all

k,21,r3-hb-s_arms,r1-hb_t+t6

k,22,r3-hb-s_arms-t1,r1-hb_t+t6-dt

k,23,r3-hb-s_arms-t1,r1-hb_t-dt-0.001

A,9,21,22,23

k,24,r3-hb-s_arms,r1+hb_t-t6

k,25,r3-hb-s_arms-t1,r1+hb_t-t6-dt

$\mathrm{k}, 26, \mathrm{r} 3-\mathrm{hb}-\mathrm{s} \_$arms-t1,r1+hb_t

A, $12,24,25,26$

$\mathrm{K}, 27, \mathrm{t}, \mathrm{r} 1-\mathrm{h} 1-\mathrm{hb} \_\mathrm{t}+\mathrm{t} 6$

$\mathrm{k}, 28, \mathrm{t}+\mathrm{t} 1, \mathrm{r} 1-\mathrm{h} 1-\mathrm{hb} \_\mathrm{t}+\mathrm{t} 6+\mathrm{dt}$

k,29,t+t1,r1-h1-hb_t

A, $14,27,28,29$

$\mathrm{K}, 30, \mathrm{t}, \mathrm{r} 1-\mathrm{h} 1+\mathrm{hb} \_\mathrm{t}-\mathrm{t} 6$

$\mathrm{k}, 31, \mathrm{t}+\mathrm{t} 1, \mathrm{r} 1-\mathrm{h} 1+\mathrm{hb} \_\mathrm{t}-\mathrm{t} 6+\mathrm{dt}$

$\mathrm{k}, 32, \mathrm{t}+\mathrm{t} 1, \mathrm{r} 1+\mathrm{hb} \_\mathrm{t}$

A, $15,30,31,32$

$\mathrm{k}, 33, \mathrm{t}, \mathrm{r} 1-\mathrm{h} 1+\mathrm{hb} \_\mathrm{t}-\mathrm{t} 2-\mathrm{t} 6$

$\mathrm{k}, 34, \mathrm{t}+\mathrm{t} 1, \mathrm{r} 1-\mathrm{h} 1+\mathrm{hb} \_\mathrm{t}-\mathrm{t} 2-\mathrm{t} 6+\mathrm{dt}$

$\mathrm{k}, 35, \mathrm{t}+\mathrm{t} 1, \mathrm{r} 1-\mathrm{h} 1-\mathrm{hb} \_\mathrm{t}+\mathrm{t} 2+\mathrm{t} 6+\mathrm{dt}$

$\mathrm{k}, 36, \mathrm{t}, \mathrm{r} 1-\mathrm{h} 1-\mathrm{hb} \_\mathrm{t}+\mathrm{t} 2+\mathrm{t} 6$

A,33,34,35,36

$\mathrm{k}, 37, \mathrm{r3}-\mathrm{hb}-\mathrm{t} 1$-s_arms,r1+hb_t-t2-t6-dt

$\mathrm{k}, 38, \mathrm{r} 3-\mathrm{hb}-\mathrm{s}$ arms,r1+hb_t-t2-t6

$\mathrm{k}, 39, \mathrm{r} 3-\mathrm{hb}-\mathrm{s} \_a r m s, \mathrm{r} 1-\mathrm{hb} \_\mathrm{t}+\mathrm{t} 2+\mathrm{t} 6$

k,40,r3-hb-t1-s_arms,r1-hb_t+t2+t6-dt

A,37,38,39,40

k,41,r3-hb-t1-s_arms,r1-t3-dt

$\mathrm{k}, 42, \mathrm{r} 3-\mathrm{hb}-\mathrm{t} 1$-s_arms,r1+t3-dt

$\mathrm{k}, 43, \mathrm{t}+\mathrm{t} 1, \mathrm{r} 1-\mathrm{h} 1+\mathrm{dt}+\mathrm{t} 3$

$\mathrm{k}, 44, \mathrm{t}+\mathrm{t} 1, \mathrm{r} 1-\mathrm{h} 1+\mathrm{dt}-\mathrm{t} 3$ 

A,41,42,43,44
ASBA,6,ALL
1fillt, 28,29,rf,, lfillt,29,47,rf,,
lfillt,35,32,rf,, lfillt,32,50,rf,, lfillt,35,34,rf,, lfillt,34,51,rf,,
lfillt, 14,25,rf,, lfillt,25,46,rf,,
!! Right side
lfillt,45,10,rf,, lfillt,10,8,rf,,
lfillt,52,36,rf,, lfillt,36,37,rf,,
lfillt,53,38,rf,, lfillt,38,37,rf,,
lfillt,44,3,rf, lfillt, 3,1,rf,,
al,5,6,11
al,27,26,30
al,42,40,54
al,59,58,60
al, 17,12,18
al,31,33,39
al,55,56,57
al,61,62,63
al,64,65,66
al,70,71,72
al,76,77,78
al,82,83,84
al,85,86,87 
al, $79,80,81$

al, $73,74,75$

al,67,68,69

lfillt, 9,24, rf,

al, $88,89,90$

AADD, all

CYL4,r3-fb-r,0,r

RECTNG,r3-hb,r3-fb-r,-r,r,

CYL4,r3+fb+r, $0, \mathrm{r}$

RECTNG,r3+r+fb,r3+hb,-r,r,

BLC5,r3-hb-t5/2,h3,t5,h4

CYL4, r3-hb-r,h3-f-r,r

RECTNG, r3-hb-2*r,r3-hb,h3-h4/2,h3-f-r,

CYL4, r3-hb-r,h3+f+r,r

RECTNG, r3-hb-2*r,r3-hb,h3+f+r,h3+h4/2,

AADD, 5,19

ASBA, 10,ALL

RECTNG,0,0.0025,r1-h1+hb_t,r1-h1+hb_t+h_act

RECTNG,0,0.02,r1-h1+hb_t+h_act,r1-h1+hb_t+h_act + load_top

BLC4,0,h2-hb,r3+hb + 0.015,2*hb

AADD, 3,5

$\mathrm{AADD}, 4,1$

ARSYM, $x$, all

AADD, 4,3

$\mathrm{AADD}, 1,2$

LCOMB, 124, 123,0

LCOMB, 123,170,0

LCOMB, 123,68,0

LCOMB, 68,20,0

LCOMB, 20,63,0

LCOMB, 74, 172,0

LCOMB, 21,169,0 
LCOMB, 16,22,0

LCOMB, 7,23,0

LCOMB, 132,133,0

\section{ET,1,PLANE82}

KEYOPT,1,3,3

KEYOPT, 1,5,0

KEYOPT, 1,6,0

r, 1,thickness

AGLUE,3,5

SMRT, 1

MSHAPE, 0,2D

CM,_Y,AREA

ASEL, , , , 1

CM,_Y1,AREA

CHKMSH,'AREA'

CMSEL,S,_Y

AMESH,_Y1

CMDELE, $Y$

CMDELE,_Y1

CMDELE,_Y2

SMRT,1

MSHAPE,0,2D

CM,_Y,AREA

ASEL, , , 2

CM,_Y1,AREA

CHKMSH,'AREA'

CMSEL,S,_Y

AMESH,_Y1

CMDELE,_Y

CMDELE,_Y1

CMDELE,_Y2

$d x=10 e-6$

DL, 110, ux,-dx

DL, 69, ux,dx

DL,20,,ALL

ACEL,0,-9.8 


\section{FINISH}

/SOL

solv

*GET,F1,NODE,1496,RF,FX

*GET,F2,NODE,2951,RF,FX

*GET,F3,NODE,2952,RF,FX

*GET,F4,NODE,2953,RF,FX

*GET,F5,NODE,2954,RF,FX

*GET,F6,NODE,2955,RF,FX

*GET,F7,NODE,2956,RF,FX

*GET,F8,NODE,2957,RF,FX

*GET,F9,NODE,2958,RF,FX

*GET,F10,NODE,2959,RF,FX

*GET,F11,NODE,2960,RF,FX

*GET,F12,NODE,2961,RF,FX

*GET,F13,NODE,2962,RF,FX

*GET,F14,NODE,2963,RF,FX

*GET,F15,NODE,1497,RF,FX

$\mathrm{F} 30=\mathrm{F} 1+\mathrm{F} 2+\mathrm{F} 3+\mathrm{F} 4+\mathrm{F} 5+\mathrm{F} 6+\mathrm{F} 7+\mathrm{F} 8$

$\mathrm{F} 31=\mathrm{F} 9+\mathrm{F} 10+\mathrm{F} 11+\mathrm{F} 12+\mathrm{F} 13+\mathrm{F} 14+\mathrm{F} 15$

FTOTAL $=F 30+F 31$

ForceMax $=2300-(19 \mathrm{E} 6) * \mathrm{dx}$

Force $=$ ForceMax-FTOTAL

/POST1

AVPRIN, 0,0 ,

ETABLE,Stress,S,EQV

ESORT,ETAB,STRESS, 0,1 , ,

*GET,StressMax,SORT,,MAX

AVPRIN, 0,0 ,

ETABLE,UY,U,Y

ESORT,ETAB,UY,0,1, ,

*GET,DMAX,SORT,,MAX

FINISH

/SOLUTION 


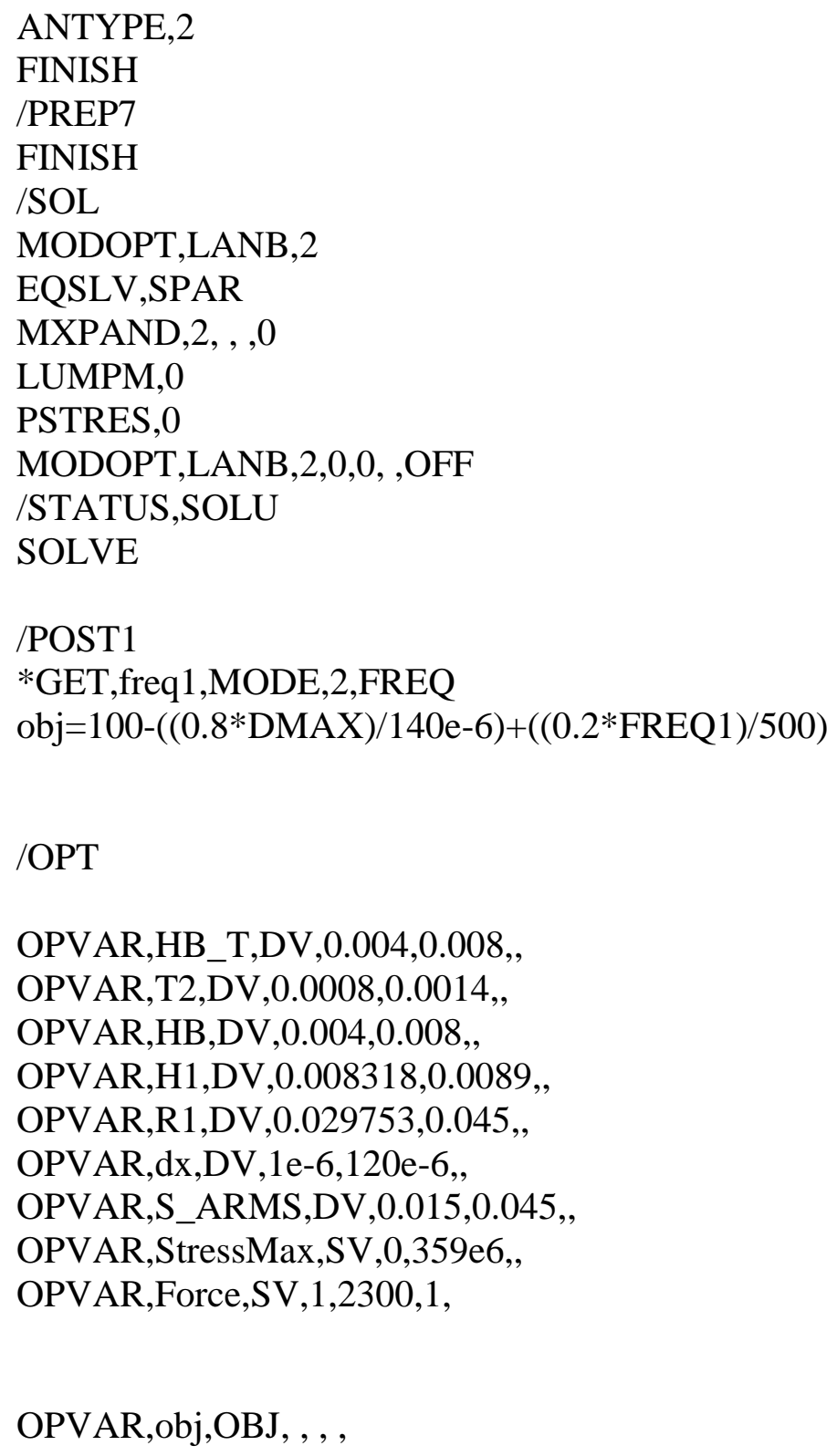

OPVAR,obj,OBJ, , , , 


\title{
APPENDIX C
}

\section{ANSYS Codes for the Unloaded XY CMDA}

\author{
$\mathrm{r} 1=0.23696 \mathrm{E}-01$ \\ $\mathrm{t} 2=0.35 \mathrm{E}-03$ \\ $\mathrm{h} 3=1.1 \mathrm{E}-02$ \\ $\mathrm{h} 1=0.25 \mathrm{E}-02$ \\ $\mathrm{fb}=0.35 \mathrm{E}-03$ \\ $\mathrm{r} 3=44 \mathrm{e}-3$ \\ $\mathrm{t}=5 \mathrm{e}-3$ \\ h2 $=-3 \mathrm{e}-3$ \\ $\mathrm{b}=6 \mathrm{e}-3$ \\ $\mathrm{hb}=2.5 \mathrm{e}-3$ \\ $\mathrm{rt}=\mathrm{r} 3+\mathrm{t}$ \\ rh1=r1-h1 \\ thickness $=10 \mathrm{e}-3$ \\ $\mathrm{rf}=0.25 \mathrm{e}-3$ \\ $\mathrm{t} 1=3 \mathrm{e}-3$ \\ $\mathrm{t} 3=0.5 \mathrm{e}-3$ \\ $\mathrm{t} 4=\mathrm{abs}(\mathrm{h} 1)$ \\ $\mathrm{t} 5=4.5 \mathrm{e}-3$ \\ $\mathrm{t} 6=(\mathrm{hb}-\mathrm{t} 2-\mathrm{t} 3) / 2$ \\ $\mathrm{r}=0.5 \mathrm{e}-3$ \\ $\mathrm{h} 4=10 \mathrm{e}-3$ \\ $\mathrm{f}=0.25 \mathrm{e}-3$ \\ $r b=0.5 e-3$ \\ $\mathrm{dt}=\mathrm{h} 1 * \mathrm{t} 1 /(\mathrm{r} 3-\mathrm{t}-\mathrm{hb})$ \\ $\mathrm{d}=0.005$ \\ $\mathrm{m}=0.0055$ \\ /prep7 \\ WPROTA,-90,0,0
}


BTOL,1e-6

BLC4,0,h2-hb,r3+hb,2*hb

RECTNG,r3-hb,r3+hb,h2-hb,r1+hb,

RECTNG,0,t,r1-h1-hb,r1-h1+hb,

k,213,(r1-hb),-1*(r3-hb)

A, $11,10,213,8$

AADD,all

$\mathrm{k}, 221, \mathrm{r} 1-\mathrm{hb}+\mathrm{t} 6,-1 *(\mathrm{r} 3-\mathrm{hb})$

$\mathrm{k}, 222, \mathrm{r} 1-\mathrm{hb}+\mathrm{t} 6-\mathrm{dt},-1 *(\mathrm{r} 3-\mathrm{hb}-\mathrm{t} 1)$

k,223,r1-hb-1.1*dt,-1*(r3-hb-t1)

A,213,221,222,223

$\mathrm{k}, 224, \mathrm{r} 1+\mathrm{hb}-\mathrm{t} 6,-1 *(\mathrm{r} 3-\mathrm{hb})$

k, 225,r1+hb-t6-dt,-1*(r3-hb-t1)

$\mathrm{k}, 226, \mathrm{r} 1+\mathrm{hb},-1 *(\mathrm{r} 3-\mathrm{hb}-\mathrm{t} 1)$

A, 8,224,225,226

K, 227,r1-h1-hb+t6,-1*t

$\mathrm{k}, 228, \mathrm{r} 1-\mathrm{h} 1-\mathrm{hb}+\mathrm{t} 6+\mathrm{dt},-1 *(\mathrm{t}+\mathrm{t} 1)$

$\mathrm{k}, 229, \mathrm{r} 1-\mathrm{h} 1-\mathrm{hb},-1 *(\mathrm{t}+\mathrm{t} 1)$

A, 10,227,228,229

K,300,r1-h1+hb-t6,-1*t

$\mathrm{k}, 301, \mathrm{r} 1-\mathrm{h} 1+\mathrm{hb}-\mathrm{t} 6+\mathrm{dt},-1 *(\mathrm{t}+\mathrm{t} 1)$

$\mathrm{k}, 302, \mathrm{r} 1+\mathrm{hb},-1 *(\mathrm{t}+\mathrm{t} 1)$

A,11,300,301,302

k,303,r1-h1+hb-t2-t6,-1*t

$\mathrm{k}, 304, \mathrm{r} 1-\mathrm{h} 1+\mathrm{hb}-\mathrm{t} 2-\mathrm{t} 6+\mathrm{dt},-1 *(\mathrm{t}+\mathrm{t} 1)$

$\mathrm{k}, 305, \mathrm{r} 1-\mathrm{h} 1-\mathrm{hb}+\mathrm{t} 2+\mathrm{t} 6+\mathrm{dt},-1 *(\mathrm{t}+\mathrm{t} 1)$

k,306,r1-h1-hb+t2+t6,-1*t

A,303,304,305,306

k,307,r1+hb-t2-t6-dt,-1*(r3-hb-t1) 


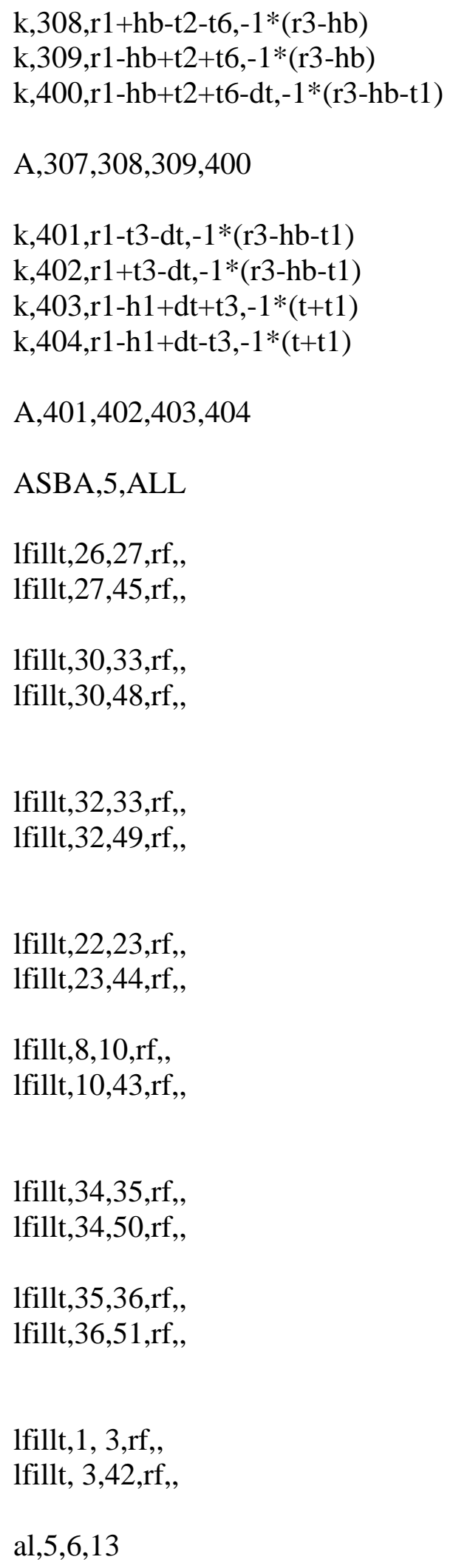


al, 14,15,21

al, $24,25,28$

al,29,31,37

al, $38,40,52$

al, $56,57,58$

al, $59,60,61$

$\mathrm{al}, 62,63,64$

al, 65,66,67

al, 68,69,70

al, $71,72,73$

al, $74,75,76$

al, $77,78,79$

al, $80,81,82$

al, $83,84,85$

al, $53,54,55$

AADD, 1,2,3,4,5,6,7,8,9

AADD,all

CYL4,r3-fb-r,0,r

RECTNG,r3-hb,r3-fb-r,-r,r,

CYL4,r3+fb+r,0,r

RECTNG,r3+r+fb,r3+hb,-r,r,

BLC5,r3-hb-t5/2,h3,t5,h4

CYL4, r3-hb-r,h3-f-r,r

RECTNG, r3-hb-2*r,r3-hb,h3-h4/2,h3-f-r,

CYL4, r3-hb-r,h3+f+r,r

RECTNG, r3-hb-2*r,r3-hb,h3+f+r,h3+h4/2,

AADD, 1,6

ASBA,11,ALL

RECTNG,0,t,r1-h1+hb,r1-h1+hb+t4,

AADD,all

ARSYM, Y,3, , , ,0,0

AADD, all

CSWPLA, 120,2,1,1, 
CSYS, 120,

AGEN, 2,2, , , ,90, , ,1

WPROTA,90,0,0

CSWPLA,90,0,1,1

CSYS,90

AGEN, ,1, , ,1*(0.0465+(r1+hb)+d),-(r3+hb)-(h2-hb)-2*hb-(r1+hb)-m,0, , ,1

blc4,r1-h1+hb+t4,-t,d+((r3+hb)-(r3-hb))+r3+hb,2*t

BLC4,r1-h1+hb+t4+(d+((r3+hb)-(r3-hb))+r3+hb),-t,-2*t,-1*((r3+hb)-t+d)

AADD,ALL

BLC4,r1-h1+hb+t4,-t,6*r,t/5

BLC4,r1-h1+hb+t4,t,6*r,-t/5

BLC4,r1-h1+hb+t4,-t+(t*1.7),6*r,-t/5

BLC4,r1-h1+hb+t4,t-(t*1.7),6*r,t/5

BLC4, $(\mathrm{d}+((\mathrm{r} 3+\mathrm{hb})-(\mathrm{r} 3-\mathrm{hb}))+\mathrm{r} 3+\mathrm{hb})+(\mathrm{r} 1-\mathrm{h} 1+\mathrm{hb}+\mathrm{t} 4)-(2 * \mathrm{t}), \mathrm{t},-6 * \mathrm{r},-\mathrm{t} / 5$

BLC4, (d+((r3+hb)-(r3-hb))+r3+hb)+(r1-h1+hb+t4)-(2*t),-t,-6*r,t/5

BLC4, $(\mathrm{d}+((\mathrm{r} 3+\mathrm{hb})-(\mathrm{r} 3-\mathrm{hb}))+\mathrm{r} 3+\mathrm{hb})+(\mathrm{r} 1-\mathrm{h} 1+\mathrm{hb}+\mathrm{t} 4)-(2 * \mathrm{t}),-\mathrm{t}+(\mathrm{t} * 1.7),-6 * \mathrm{r},-\mathrm{t} / 5$

BLC4, $(\mathrm{d}+((\mathrm{r} 3+\mathrm{hb})-(\mathrm{r} 3-\mathrm{hb}))+\mathrm{r} 3+\mathrm{hb})+(\mathrm{r} 1-\mathrm{h} 1+\mathrm{hb}+\mathrm{t} 4)-(2 * \mathrm{t}), \mathrm{t}-(\mathrm{t} * 1.7),-6 * \mathrm{r}, \mathrm{t} / 5$

BLC4,r1-h1+hb+t4+(d+((r3+hb)-(r3-hb))+r3+hb),-t,-t/5,-6*r

BLC4, (r1-h1+hb+t4+(d+((r3+hb)-(r3-hb))+r3+hb))-2*t,-t,t/5,-6*r

BLC4, (r1-h1+hb+t4+(d+((r3+hb)-(r3-hb))+r3+hb))-(2*t)+t*1.7,-t,-t/5,-6*r

BLC4,(r1-h1+hb+t4+(d+((r3+hb)-(r3-hb))+r3+hb))-t*1.7,-t,t/5,-6*r

BLC4,r1-h1+hb+t4+(d+((r3+hb)-(r3-hb))+r3+hb),-1*((r3+hb)-t+(h2-hb)-(r1-h1+hb+t4))-t-2*hb$(\mathrm{r} 1+\mathrm{hb})-\mathrm{m},-\mathrm{t} / 5,6 * \mathrm{r}$

BLC4, (r1-h1+hb+t4+(d+((r3+hb)-(r3-hb))+r3+hb))-2*t,-1*((r3+hb)-t+(h2-hb)-(r1-h1+hb+t4))-t$2 * h b-(r 1+h b)-m, t / 5,6 * r$

BLC4, (r1-h1+hb+t4+(d+((r3+hb)-(r3-hb))+r3+hb))-(2*t)+t*1.7,-1*((r3+hb)-t+(h2-hb)-(r1$\mathrm{h} 1+\mathrm{hb}+\mathrm{t} 4))-\mathrm{t}-2 * \mathrm{hb}-(\mathrm{r} 1+\mathrm{hb})-\mathrm{m},-\mathrm{t} / 5,6 * \mathrm{r}$

BLC4,(r1-h1+hb+t4+(d+((r3+hb)-(r3-hb))+r3+hb))-t*1.7,-1*((r3+hb)-t+(h2-hb)-(r1-h1+hb+t4))$\mathrm{t}-2 * \mathrm{hb}-(\mathrm{r} 1+\mathrm{hb})-\mathrm{m}, \mathrm{t} / 5,6 * \mathrm{r}$ 
ASBA,5,ALL

LSTR， 294， 311

LSTR, 311, 316

LSTR, 316, 301

LSTR, 301, 294

LSTR, 332, 329

LSTR, 329, 342

LSTR, 342, 347

LSTR, 347, 332

AL,13,167,292,293

AL,297,298,299,300

ASBA, 18,ALL

lfillt,307,306,rf,, lfillt,309,306,rf,,

lfillt,317,316,rf,, lfillt,315,316,rf,,

lfillt,319,318,rf,, lfillt,321,318,rf,,

lfillt,313,312,rf,, lfillt,362,312,rf,

lfillt,291,289,rf,, lfillt,354,289,rf,,

lfillt,295,296,rf,, lfillt,301,296,rf,,

lfillt,303,302,rf,, lfillt,305,302,rf,,

lfillt, 152,166,rf,, lfillt,355,166,rf,,

lfillt,363,327,rf,, lfillt, 326,327,rf,,

lfillt,336,337,rf,, 


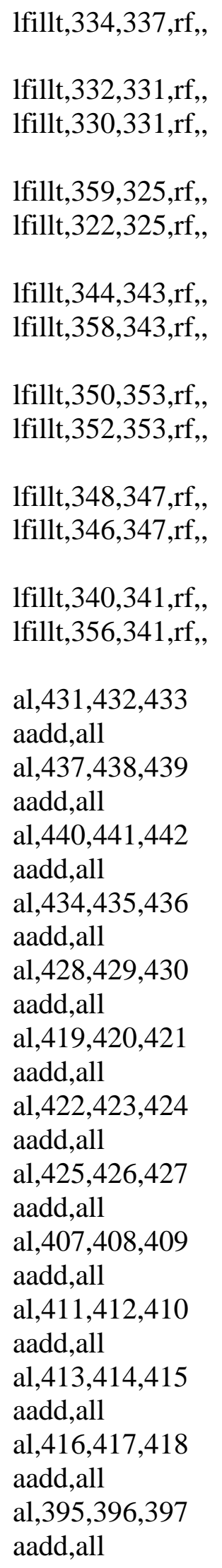




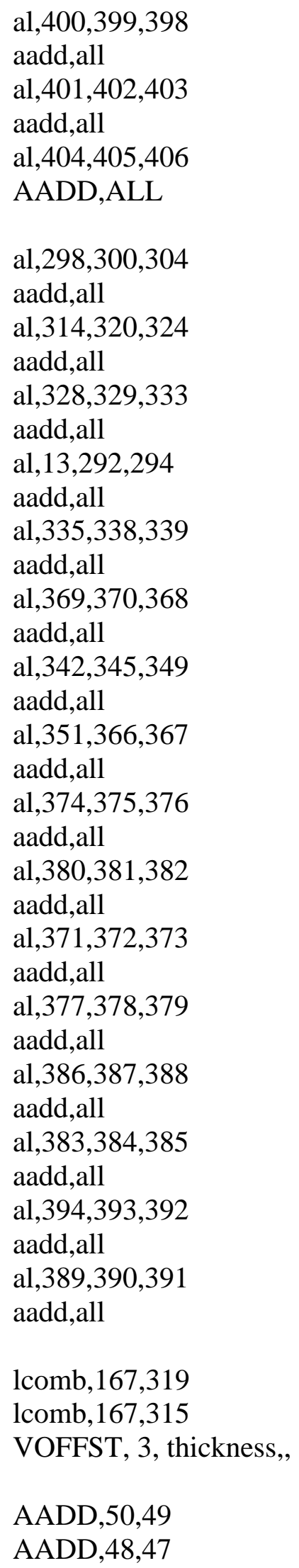


AADD,379,49

AADD, 111,112

AADD, 113,114

AADD, 48,49

ET,1,SOLID186

KEYOPT, 1,10,0

KEYOPT,1,6,0

mp,ex,1,200e9

mp,nuxy, 1,0.285

mp,dens, 1,8000

SMRT,6

MSHAPE,1,3D

CM,_Y,VOLU

VSEL, , , 1

CM,_Y1,VOLU

CHKMSH,'VOLU'

CMSEL,S,_Y

VMESH,_Y1

CMDELE,_Y

CMDELE,_Y1

CMDELE,_Y2

SHPP,WARN

$d x=0.1 E-04$

DA,365,ux,-dx

DA,328,ux,dx

DA,306,uy,dx

DA,269,uy,-dx

DA,50,ALL

DA,47,ALL

ACEL,,-9.8

FINISH

/SOL

solv 


\section{FINISH}

/POST1

AVPRIN,0,0,

ETABLE,Stress,S,EQV

ESORT,ETAB,STRESS,0,1, ,

*GET,StressMax,SORT,,MAX

AVPRIN,0,0,

ETABLE,UY,U,Y

ESORT,ETAB,UY,0,1, ,

*GET,DMAX,SORT,,MAX

DMAX1=0.01-DMAX

*GET,F1,NODE,703,RF,FX

*GET,F2,NODE, 14692,RF,FX

*GET,F3,NODE,284,RF,FX

*GET,F4,NODE, 14691,RF,FX

*GET,F5,NODE,3188,RF,FX

*GET,F6,NODE,35926,RF,FX

*GET,F7,NODE,35930,RF,FX

*GET,F8,NODE,35932,RF,FX

*GET,F9,NODE, 1725,RF,FX

*GET,F10,NODE, 1726,RF,FX

*GET,F11,NODE, 285,RF,FX

*GET,F12,NODE,35928,RF,FX

*GET,F13,NODE,35929,RF,FX

*GET,F14,NODE,35933,RF,FX

*GET,F15,NODE, 1727,RF,FX

*GET,F16,NODE,3186,RF,FX

*GET,F17,NODE,35934,RF,FX

*GET,F18,NODE,35931,RF,FX

*GET,F19,NODE, 14690,RF,FX

*GET,F20,NODE,35933,RF,FX

*GET,F21,NODE, 14689,RF,FX

*GET,F22,NODE,3187,RF,FX

*GET,F23,NODE,702,RF,FX

$\mathrm{F} 24=\mathrm{F} 1+\mathrm{F} 2+\mathrm{F} 3+\mathrm{F} 4+\mathrm{F} 5+\mathrm{F} 6+\mathrm{F} 7+\mathrm{F} 8+\mathrm{F} 9$

$\mathrm{F} 25=\mathrm{F} 10+\mathrm{F} 11+\mathrm{F} 12+\mathrm{F} 13+\mathrm{F} 14+\mathrm{F} 15+\mathrm{F} 16$

$\mathrm{F} 26=\mathrm{F} 17+\mathrm{F} 18+\mathrm{F} 19+\mathrm{F} 20+\mathrm{F} 21+\mathrm{F} 22 * \mathrm{~F} 23$

Ftotal $=\mathrm{F} 23+\mathrm{F} 24+\mathrm{F} 25$

ForceMax $=1000-(20 \mathrm{E} 6) * \mathrm{dx}$ 
Force $=$ ForceMax-Ftotal

/OPT

OPVAR,R1,DV,0.023696,0.0257,, OPVAR,T2,DV, $0.3 \mathrm{e}-3,0.5 \mathrm{e}-3$,, OPVAR,H3,DV,1.05E-02,1.25E-02 , OPVAR,H1,DV,2e-3,3e-3,

OPVAR,FB,DV,0.25e-3,0.4e-3, OPVAR,dx,DV,9.999E-6,10e-6, OPVAR,StressMax,SV,0,703e6,100, OPVAR,Force,SV,1,1000,0.1,

OPVAR,DMAX1,OBJ, , , , 


\section{APPENDIX D}

CATIA Drawing of the Proposed XY Planar Motion Generator CMDA

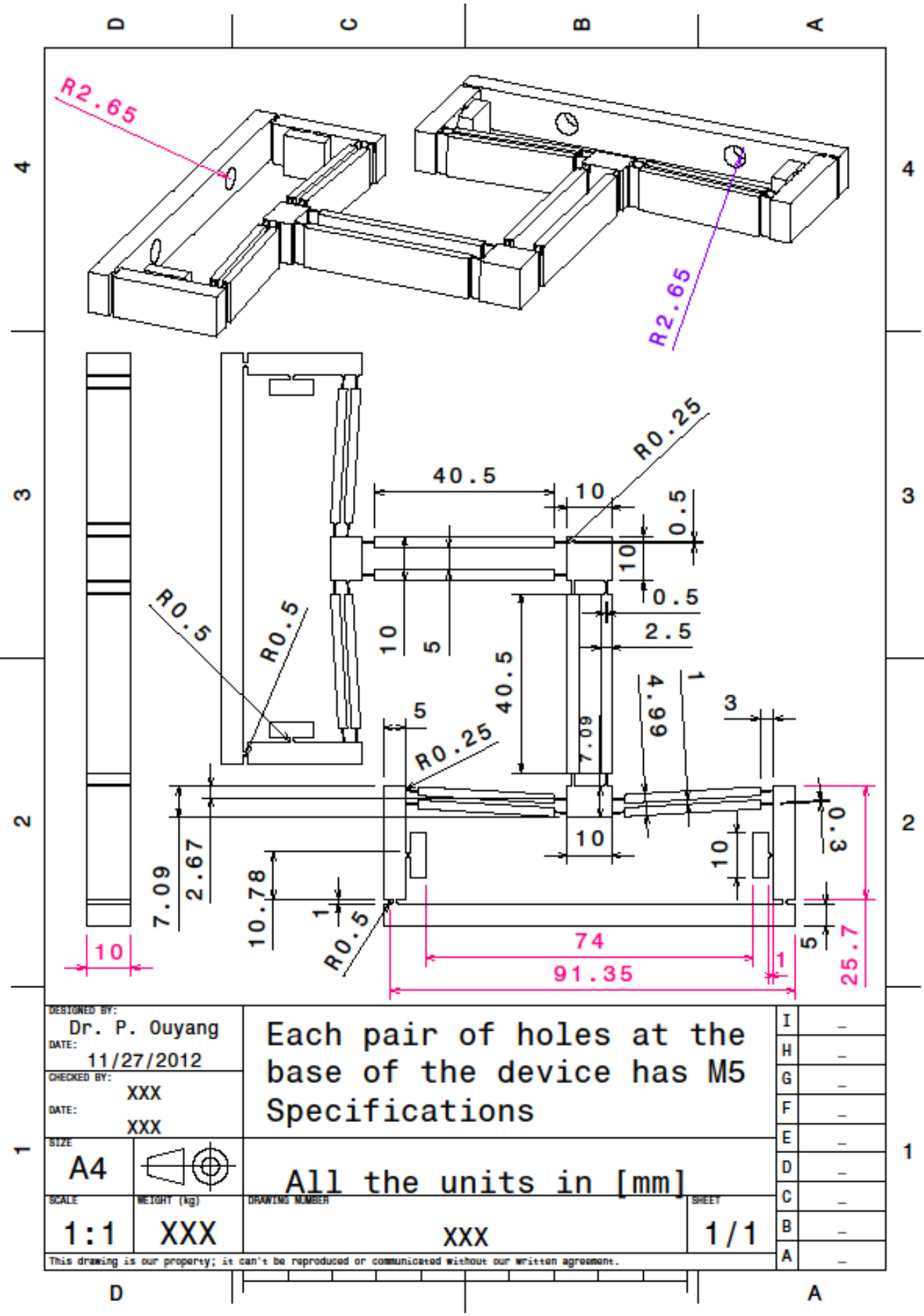

